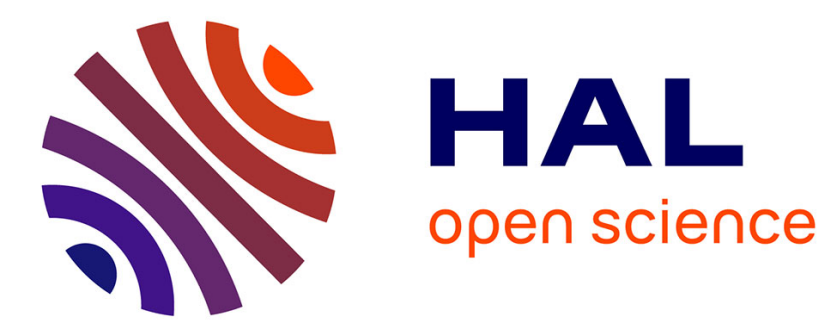

\title{
The recovery of a recessive allele in a Mendelian diploid model
}

\author{
Anton Bovier, Loren Coquille, Rebecca Neukirch
}

\section{To cite this version:}

Anton Bovier, Loren Coquille, Rebecca Neukirch. The recovery of a recessive allele in a Mendelian diploid model. Journal of Mathematical Biology, In press, 10.1007/s00285-018-1240-z . hal-01485246

\section{HAL Id: hal-01485246 \\ https://hal.science/hal-01485246}

Submitted on 8 Mar 2017

HAL is a multi-disciplinary open access archive for the deposit and dissemination of scientific research documents, whether they are published or not. The documents may come from teaching and research institutions in France or abroad, or from public or private research centers.
L'archive ouverte pluridisciplinaire HAL, est destinée au dépôt et à la diffusion de documents scientifiques de niveau recherche, publiés ou non, émanant des établissements d'enseignement et de recherche français ou étrangers, des laboratoires publics ou privés. 


\title{
THE RECOVERY OF A RECESSIVE ALLELE IN A MENDELIAN DIPLOID MODEL
}

\author{
ANTON BOVIER, LOREN COQUILLE, AND REBECCA NEUKIRCH
}

\begin{abstract}
We study the large population limit of a stochastic individual-based model which describes the time evolution of a diploid hermaphroditic population reproducing according to Mendelian rules. In [25] it is proved that sexual reproduction allows unfit alleles to survive in individuals with mixed genotype much longer than they would in populations reproducing asexually. In the present paper we prove that this indeed opens the possibility that individuals with a pure genotype can reinvade in the population after the appearance of further mutations. We thus expose a formal description of a mechanism by which a recessive allele can re-emerge in a population. This can be seen as a statement of genetic robustness exhibited by diploid populations performing sexual reproduction.
\end{abstract}

\section{INTRODUCTION}

In population genetics, the study of Mendelian diploid models of fixed population size began more than a century ago (see e.g. [2, 11, 13, 14, 16, 17, 24, 27, 28]), while their counterparts of variable population size models were studied in the context of adaptive dynamics from 1999 onwards [20]. The approach of adaptive dynamics is to introduce competition kernels to regulate the population size instead of maintaining it constant, see [19, 21, 22].

Stochastic individual-based versions of these models appeared in the 1990s, see [3-6, 12, 15]. They assume single events of reproduction, mutation, natural death, and death by competition happen at random times to each individual in the population. An important and interesting feature of these models is that different limiting processes on different time-scales appear as the carrying capacity tends to infinity while mutation rates and mutation step-size tend to zero (see [1, 3, 6, 12,23]). One of the major results in this context is the convergence of a properly rescaled process to the so called Trait Substitution Sequence (TSS) process, which describes the evolution of a monomorphic population as a jump process between monomorphic equilibria. More generally, Champagnat and Méléard [6] obtained the convergence to a Polymorphic Evolution Sequence (PES), where jumps occur between equilibria that may include populations that have multiple co-existing phenotypes. The appearance of co-existing phenotypes is, however, exceptional and happens only at so-called evolutionary singularities. From a biological point of view, this is somewhat unsatisfactory, as it apparently fails to explain the biodiversity seen in real biological systems.

1991 Mathematics Subject Classification. 60K35,92D25,60J85.

We acknowledge financial support from the German Research Foundation (DFG) through the Hausdorff Center for Mathematics, the Cluster of Excellence ImmunoSensation, and the Priority Programme SPP1590 Probabilistic Structures in Evolution. L.C. has been partially supported by the LabEx PERSYVAL-Lab (ANR-11-LABX-0025-01) through the Exploratory Project CanDyPop and by the Swiss National Science Foundation through the grant No. P300P2_161031.

We would like to thank Pierre Collet and Vincent Beffara for their help on the theory of dynamical systems and fruitful discussions. 
Most of the models considered in this context assume haploid populations with a-sexual reproduction. One exception is the paper [7] by Collet, Méléard and Metz in 2013, and then a series of papers by Coron and co-authors [8-10]. In [7], the Trait Substitution Sequence is derived in a Mendelian diploid model under the assumption that the fitter mutant allele and the resident allele are co-dominant.

The main reason why both in haploid models and in the model considered in [7] the evolution along monomorphic populations is typical is that the time scales for the fixation of a new trait and the extinction of the resident trait are the same (both of order $\ln K$ ) (unless some very special fine-tuning of parameters occurs that allows for co-existence). This precludes (at least in the rare mutation scenarios considered) that an initially less fit trait survives long enough until after possibly several new mutations occurred that might create a situation where this trait may become fit again and recover.

In a follow-up paper to [7], two of the present authors [25], it was shown that, if instead one assumes that the resident allele is recessive, the time to extinction of this allele is dramatically increased. This will be discussed in detail in Section 1.2 and paves the way for the appearance of a richer limiting process.

The general framework in [7] and [25] is the following. Each individual is characterised by a reproduction and death rate which depend on a phenotypic trait determined by its genotype, which here is determined by two alleles (e.g. $A$ and $a$ ) on one single locus. The evolution of the trait distribution of the three genotypes $a a, a A$ and $A A$ is studied under the action of (1) heredity, which transmits traits to new offsprings according to Mendelian rules, (2) mutation, which produces variations in the trait values in the population onto which selection is acting, and (3) of competition for resources between individuals.

The paper [25] proves that sexual reproduction allows unfit alleles to survive in individuals with mixed genotype much longer than they would in populations reproducing asexually. This opens the possibility that while this allele is still alive in the population, the appearance of new mutants alters the fitness landscape in such a way that is favourable for this allele and allow it to reinvade in the population, leading to a new equilibrium with co-existing phenotypes. The goal of this paper is to rigorously prove that such a scenario indeed occurs under fairly natural assumptions.

1.1. The stochastic model. The individual-based microscopic Mendelian diploid model is a non-linear birth-and-death process. We consider a model for a population of a finite number of hermaphroditic individuals which reproduce sexually. Each individual $i$ is characterised by two alleles, $u_{1}^{i} u_{2}^{i}$, taken from some allele space $\mathcal{U} \subset \mathbb{R}$. These two alleles define the genotype of the individual $i$. We suppress parental effects, which means that we identify individuals with genotype $u_{1} u_{2}$ and $u_{2} u_{1}$. Each individual has a Mendelian reproduction rate with possible mutations and a natural death rate. Moreover, there is an additional death rate due to ecological competition with the other individuals in the population. Let

$$
\begin{aligned}
& f_{u_{1} u_{2}} \in \mathbb{R}_{+} \\
& D_{u_{1} u_{2}} \in \mathbb{R}_{+} \\
& K \in \mathbb{N} \\
& \frac{c_{u_{1} u_{2}, v_{1} v_{2}}}{K} \in \mathbb{R}_{+} \\
& R_{u_{1} u_{2}}\left(v_{1} v_{2}\right) \in\{0,1\} \\
& \mu_{K} \in \mathbb{R}_{+}
\end{aligned}
$$

the per capita birth rate (fertility) of an individual with genotype $u_{1} u_{2}$. the per capita natural death rate of an individual with genotype $u_{1} u_{2}$. the carrying capacity, a parameter which scales the population size. the competition effect felt by an individual with genotype $u_{1} u_{2}$ from an individual of genotype $v_{1} v_{2}$. the reproductive compatibility of the genotype $v_{1} v_{2}$ with $u_{1} u_{2}$ the mutation probability per birth event. Here it is independent of the genotype. 
$m(u, d h)$

mutation law of a mutant allelic trait $u+h \in \mathcal{U}$, born from an individual with allelic trait $u$.

Scaling the competition function $c$ down by a factor $1 / K$ amounts to scaling the population size to order $K$. We are interested in asymptotic results when $K$ is large. We assume rare mutation, i.e. $\mu_{K} \ll 1$. If a mutation occurs at a birth event, only one allele changes from $u$ to $u+h$ where $h$ is a random variable with law $m(u, d h)$.

At any time $t$, there is a finite number, $N_{t}$, of individuals, each with genotype in $\mathcal{U}^{2}$. We denote by $u_{1}^{1}(t) u_{2}^{1}(t), \ldots, u_{1}^{N_{t}}(t) u_{2}^{N_{t}}(t)$ the genotypes of the population at time $t$. The population, $v_{t}$, at time $t$ is represented by the rescaled sum of Dirac measures on $\mathcal{U}^{2}$,

$$
v_{t}=\frac{1}{K} \sum_{i=1}^{N_{t}} \delta_{u_{1}^{i}(t) u_{2}^{i}(t)} .
$$

Formally, $v_{t}$ takes values in the set of re-scaled point measures

$$
\mathcal{M}^{K}=\left\{\frac{1}{K} \sum_{i=1}^{n} \delta_{u_{1}^{i} u_{2}^{i}} \mid n \geq 0, u_{1}^{1} u_{2}^{1}, \ldots, u_{1}^{n} u_{2}^{n} \in \mathcal{U}^{2}\right\},
$$

on $\mathcal{U}^{2}$, equipped with the vague topology. Define $\langle v, g\rangle$ as the integral of the measurable function $g: \mathcal{U}^{2} \rightarrow \mathbb{R}$ with respect to the measure $v \in \mathcal{M}^{K}$. Then $\left\langle v_{t}, \mathbb{1}\right\rangle=\frac{N_{t}}{K}$ and for any $u_{1} u_{2} \in \mathcal{U}^{2}$, the positive number $\left\langle v_{t}, \mathbb{1}_{u_{1} u_{2}}\right\rangle$ is called the density at time $t$ of the genotype $u_{1} u_{2}$. The generator of the process is defined as in [7]: first we define, for the genotypes $u_{1} u_{2}, v_{1} v_{2}$ and a point measure $v$, the Mendelian reproduction operator:

$$
\begin{aligned}
& \left(A_{u_{1} u_{2}, v_{1} v_{2}} F\right)(v) \\
& \quad=\frac{1}{4}\left[F\left(v+\frac{\delta_{u_{1} v_{1}}}{K}\right)+F\left(v+\frac{\delta_{u_{1} v_{2}}}{K}\right)+F\left(v+\frac{\delta_{u_{2} v_{1}}}{K}\right)+F\left(v+\frac{\delta_{u_{2} v_{2}}}{K}\right)\right]-F(v),
\end{aligned}
$$

and the Mendelian reproduction-cum-mutation operator:

$$
\begin{aligned}
\left(M_{u_{1} u_{2}, v_{1} v_{2}} F\right)(v)=\frac{1}{8} & \int_{\mathbb{R}}\left[\left(F\left(v+\frac{\delta_{u_{1}+h, v_{1}}}{K}\right)+F\left(v+\frac{\delta_{u_{1}+h, v_{2}}}{K}\right)\right) m\left(u_{1}, h\right)\right. \\
& +\left(F\left(v+\frac{\delta_{u_{2}+h, v_{1}}}{K}\right)+F\left(v+\frac{\delta_{u_{2}+h, v_{2}}}{K}\right)\right) m\left(u_{2}, h\right) \\
& +\left(F\left(v+\frac{\delta_{u_{1}, v_{1}+h}}{K}\right)+F\left(v+\frac{\delta_{u_{2}, v_{1}+h}}{K}\right)\right) m\left(v_{1}, h\right) \\
& \left.+\left(F\left(v+\frac{\delta_{u_{1}, v_{2}+h}}{K}\right)+F\left(v+\frac{\delta_{u_{2}, v_{2}+h}}{K}\right)\right) m\left(v_{2}, h\right)\right] d h-F(v) .
\end{aligned}
$$

The process $\left(v_{t}\right)_{t \geq 0}$ is then a $\mathcal{M}^{K}$-valued Markov process with generator $L^{K}$, given for any bounded measurable function $F: \mathcal{M}^{K} \rightarrow \mathbb{R}$ by:

$$
\begin{aligned}
\left(L^{K} F\right)(v) & \\
= & \int_{\mathcal{U}^{2}}\left(D_{u_{1} u_{2}}+\int_{\mathcal{U}^{2}} c_{u_{1} u_{2}, v_{1} v_{2}} v\left(d\left(v_{1} v_{2}\right)\right)\right)\left(F\left(v-\frac{\delta_{u_{1} u_{2}}}{K}\right)-F(v)\right) K v\left(d\left(u_{1} u_{2}\right)\right) \\
& +\int_{\mathcal{U}^{2}}\left(1-\mu_{K}\right) f_{u_{1} u_{2}}\left(\int_{\mathcal{U}^{2}} \frac{f_{v_{1} v_{2}} R_{u_{1} u_{2}}\left(v_{1} v_{2}\right)}{\left\langle v R_{u_{1} u_{2}}, f\right\rangle}\left(A_{u_{1} u_{2}, v_{1} v_{2}} F\right)(v) v\left(d\left(v_{1} v_{2}\right)\right)\right) K v\left(d\left(u_{1} u_{2}\right)\right) \\
& +\int_{\mathcal{U}^{2}} \mu_{K} f_{u_{1} u_{2}}\left(\int_{\mathcal{U}^{2}} \frac{f_{v_{1} v_{2}} R_{u_{1} u_{2}}\left(v_{1} v_{2}\right)}{\left\langle v R_{u_{1} u_{2}}, f\right\rangle}\left(M_{u_{1} u_{2}, v_{1} v_{2}} F\right)(v) v\left(d\left(v_{1} v_{2}\right)\right)\right) K v\left(d\left(u_{1} u_{2}\right)\right) .
\end{aligned}
$$


The first non-linear term describes the competition between individuals. The second and last linear terms describe the birth with and without mutation. There, $f_{u_{1} u_{2}} \frac{f_{v_{1} v_{2}} R_{u_{1} u_{2}}\left(v_{1} v_{2}\right)}{K\left\langle v_{u_{1} u_{2}}, f\right\rangle}$ is the reproduction rate of an individual with genotype $u_{1} u_{2}$ with an individual with genotype $v_{1} v_{2}$. Note that $v R_{u_{1} u_{2}}$ is the population restricted to the pool of potential partners of an individual of genotype $u_{1} u_{2}$.

For all $u_{1} u_{2}, v_{1} v_{2} \in \mathcal{U}^{2}$, we make the following Assumptions (A):

(A1) The functions $f, D$ and $c$ are measurable and bounded, which means that there exists $\bar{f}, \bar{D}, \bar{c}<\infty$ such that

$$
0 \leq f_{u_{1} u_{2}} \leq \bar{f}, \quad 0 \leq D_{u_{1} u_{2}} \leq \bar{D} \quad \text { and } \quad 0 \leq c_{u_{1} u_{2}, v_{1} v_{2}} \leq \bar{c} .
$$

(A2) $f_{u_{1} u_{2}}-D_{u_{1} u_{2}}>0$ and there exists $\underline{c}>0$ such that $\underline{c} \leq c_{u_{1} u_{2}, v_{1} v_{2}}$.

(A3) There exists a function, $\bar{m}: \mathbb{R} \rightarrow \mathbb{R}_{+}$, such that $\bar{\int} \bar{m}(h) d h<\infty$ and $m(u, h) \leq \bar{m}(h)$ for any $u \in \mathcal{U}$ and $h \in \mathbb{R}$.

For fixed $K$, under the Assumptions (A1)+(A3) and assuming that $\mathbb{E}\left(\left\langle v_{0}, \mathbb{1}\right\rangle\right)<\infty$, Fournier and Méléard [15] have shown existence and uniqueness in law of a process with infinitesimal generator $L^{K}$. For $K \rightarrow \infty$, under mild restrictive assumptions, they prove the convergence of the process $v^{K}$ in the space $\mathbb{D}\left(\mathbb{R}_{+}, \mathcal{M}^{K}\right)$ of càdlàg functions from $\mathbb{R}^{+}$to $\mathcal{M}_{K}$, to a deterministic process, which is the solution to a non-linear integro-differential equation. Assumption (A2) ensures that the population does not tend to infinity in finite time or becomes extinct too fast.

1.2. Previous works. Consider the process starting with a monomorphic $a a$-population, with one additional mutant individual of genotype $a A$. Assume that the phenotype difference between the mutant and the resident population is small. The phenotype difference is assumed to be a slightly smaller death rate compared to the resident population, namely:

$$
D_{a a}=D, \quad D_{a A}=D-\Delta .
$$

for some small enough $\Delta>0$. The mutation probability for an individual with genotype $u_{1} u_{2}$ is given by $\mu_{K}$. Hence, the time until the next mutation in the whole population is of order $\frac{1}{K \mu_{K}}$. Now assume that the demographic parameters introduced in Section 1.1 depend continuously on the phenotype. In particular, they are the same for individuals bearing the same phenotype.

In [7] it is proved that if the two alleles $a$ and $A$ are co-dominant and if the allele $A$ is slightly fitter than the allele $a$, namely

$$
D_{a a}=D, \quad D_{a A}=D-\Delta, \quad D_{A A}=D-2 \Delta,
$$

then in the limit of large population and rare mutations $\left(\ln K \ll \frac{1}{\mu_{K} K} \ll e^{V K}\right.$ for some $V>$ 0 ), the suitably time-rescaled process converges to the TSS model of adaptive dynamics, essentially as shown in [3] in the haploid case. In particular, the genotypes containing the unfit allele $a$ decay exponentially fast after the invasion of $A A$ (see Figure 1 ).

If in place of co-dominance we assume, as in [25], that the fittest phenotype $A$ is dominant, namely

$$
D_{a a}=D, \quad D_{a A}=D-\Delta, \quad D_{A A}=D-\Delta,
$$

then this has a dramatic effect on the evolution of the population and, in particular, leads to a much prolonged survival of the unfit phenotype $a a$. Indeed, it was know for some time (see e.g. [24]) that in this case the unique stable fixpoint $\left(0,0, \bar{n}_{A A}\right)$ corresponding to a monomorphic $A A$ population is degenerate, i.e. its Jacobian matrix has zero-eigenvalue. 
This implies that in the deterministic system, the $a a$ and $a A$ populations decay in time only polynomially fast to zero, namely like $1 / t^{2}$ and $1 / t$, respectively. This is in contrast to the exponential decay in the co-dominant scenario (see Figure 11). In [25] it was shown that the deterministic system remains a good approximation of the stochastic system as long as the size of the $a A$ population remains much larger than $K^{1 / 2}$ and therefore that the $a$-allele survives for a time of order at least $K^{1 / 2-\alpha}$, for any $\alpha>0^{1}$ Note that this statement is a non trivial fact, since it is not a consequence of the law of large numbers, because the time window diverges as $K$ grows. In summary, the unfit recessive $a$-allele survives in the population much longer due to the slow decay of the $a A$-population.
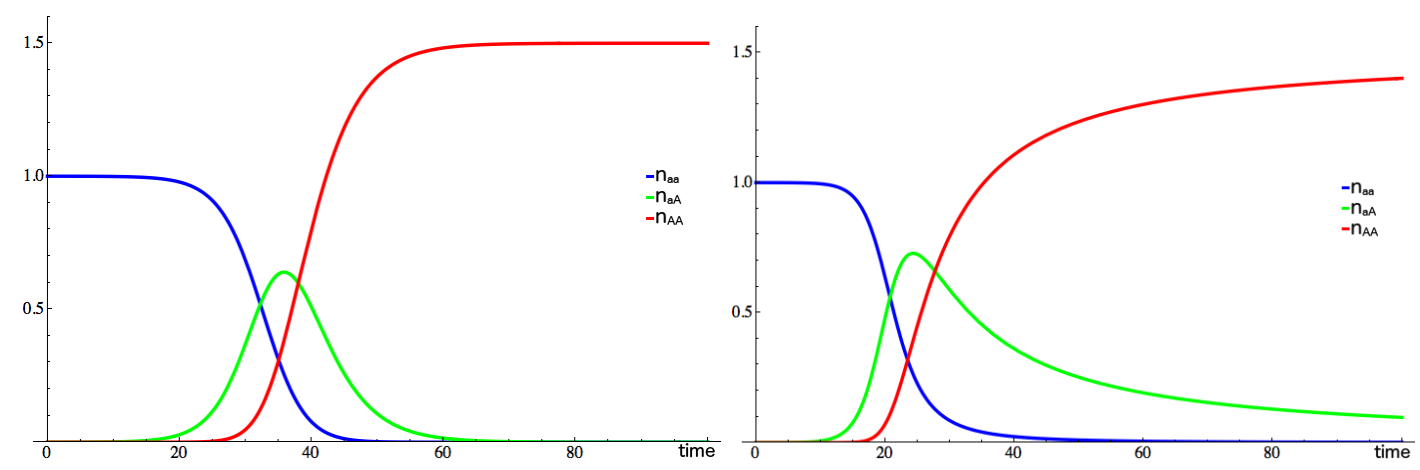

FIGURE 1. Evolution of the model from a resident $a a$ population at equilibrium with a small amount of mutant $a A$, and when the alleles $a$ and $A$ are co-dominant (left) or when the mutant phenotype $A$ is dominant (right).

It is argued in [25] that if we choose the mutation time scale in such a way that there remain enough $a$-alleles in the population when a new mutation occurs, i.e.

$$
\ln K \ll \frac{1}{\mu_{K} K} \ll K^{1 / 2-\alpha} \text { as } K \rightarrow \infty \text {, for some } \alpha>0,
$$

and if the new mutant can coexist with the unfit $a a$-individuals, then the $a a$-population can potentially recover. This is the starting point of our paper.

1.3. Goal of the paper. The goal of this paper is to show that under reasonable hypothesis, the prolonged survival of the $a$-allele after the invasion of the $A$-allele can indeed lead to a recovery of the $a a$-type. To do this, we assume that there will occur a new mutant allele, $B$, that on the one hand has a higher fitness than the $A A$-phenotype but that (for simplicity) has no competition with the $a a$-type. The possible genotypes after this mutation are $a a, a A, A A, a B, A B$, and $B B$, so that even for the deterministic system we have now to deal with a 6-dimensional dynamical system whose analysis if far from simple.

Under the assumption of dominance of the fittest phenotype, and mutation rate satisfying (1.10), we consider the model described in Section 1.1 starting at the time of the second mutation, that is (with probability converging to 1 as $K \rightarrow \infty$ ) the $A A$ population being close to its equilibrium and the $a A$ population having decreased to a size of order $K \mu_{K}$, while the $a a$-population is of the order of the square of the $a A$-population. We assume that there just occurred a mutation to a fitter (and most dominant) allele $B$ : we thus start with a quantity $\frac{1}{K}$ of genotype $A B$. We will start with a population where $A A$ is close

\footnotetext{
${ }^{1}$ In $\left[25\right.$ ] only state that survival occurs up to time $K^{1 / 4-\alpha}$. However, taking into account that it is really only the survival of the $a A$-population that needs to be ensured, one can easily improve this to $K^{1 / 2-\alpha}$.
} 
to its equilibrium, the populations of $a a$ and $a A$ are already small (of order $\varepsilon^{2}$ and $\varepsilon$ ), and by mutation a single individual of genotype $A B$ appears.

By using well known techniques [3,6], we know that the $A B$-population behaves as a super-critical branching process and reaches the level $\varepsilon$ with positive probability in a time of order $\ln K$, without perturbing the 3 -system $(a a, a A, A A)$.

We see in numerical solutions to the deterministic system that a reduced fertility together with a reduced competition between $a$ and $B$ phenotypes constitutes a sufficient condition for the recovery of the $a a$-population. For simplicity and in order to prove rigorous results, we suppose that there can be no reproduction between individuals of phenotypes $a$ and $B$, nor competition between them, and we reduce the number of remaining parameters as much as possible (see Section 2). We study the deterministic system which corresponds to the large population limit of the stochastic counterpart, and we show that (for an initial quantity $\varepsilon$ of $a A, \varepsilon^{2}$ of $a a$ and $\varepsilon^{3}$ of $A B$ ) the system converges to a fixed point denoted by $p_{a B}$ consisting of the two coexisting populations $a a$ and $B B$. If no further assumptions are made, we will show that the number of individuals bearing an $a$ allele decreases to level $\varepsilon^{1+\Delta /(1-\Delta)}$ (where $\Delta$ is defined in $(1.7)$ ) before $a a$ grows and stabilises at order 1 .

If $\Delta<\frac{\alpha}{1-2 \alpha}$, this control on the $a$ allele is in principle sufficient in order for the stochastic system to exhibit the recovery of $a a$ with positive probability in the large population limit. Indeed, if the mutation time is of order $K^{\frac{1}{2}-\alpha}$, then the initial amount of $a a$ and $a A$ genotypes is close to the typical fluctuations of those populations. Following the heuristics of [25] (although our six-dimensional stochastic process is surely much more tedious to study), the deterministic system should constitute a good approximation of the process if the typical fluctuations of populations containing an $a$ allele do not bring them to extinction. If $\Delta<\frac{\alpha}{1-2 \alpha}$ this ensures that the population containing an $a$ allele is not falling below order $K^{-1 / 2}$ at any time.

In order to go deeper and control the speed of recovery of the aa-population, we look for a parameter regime which ensures that the $a a$-population always grows after the invasion of $B$. Ensuring this lower bound on $a a$ is not trivial at all, and the solution we found is to introduce an additional parameter $\eta$, which lowers the competition between the $a A$ and $B B$ populations, compared to the one between $A A$ and $B B$. Note that the competition does not depend only on the phenotype, and can be interpreted as a refinement of a phenotypic competition for resources: the strength (or ability to get resources) of an individual not only depends on its phenotype but also on the dominance of its genotype. We show that for $\eta$ larger than some positive value (of order $\Delta$ ), the $a a$ population always grows after the invasion of $B$. The time of convergence to the coexistence fixed point is thus lowered, see Figure 5. Moreover, we point out the existence of a bifurcation: for $\eta$ larger than some threshold, the co-existence fixed point $p_{a B}$ becomes unstable and the system converges to another fixed point where all populations coexist.

Our contribution is a formal description of a mechanism by which a recessive allele can re-emerge in a population. This can be seen as a statement of genetic robustness exhibited by diploid populations performing sexual reproduction.

The structure of the paper is the following. In Section 2 we describe our assumptions on the parameters of the model, and compute the large population limit; in Section 3 we present our results on the evolution of the deterministic system towards the co-existence fixed point $p_{a B}$, and we give a heuristic of the proof. Section 4 is dedicated to the proof of these results.

Notation. We write $x=\Theta(y)$ whenever $x=O(y)$ and $y=O(x)$ as $\varepsilon \rightarrow 0$. 


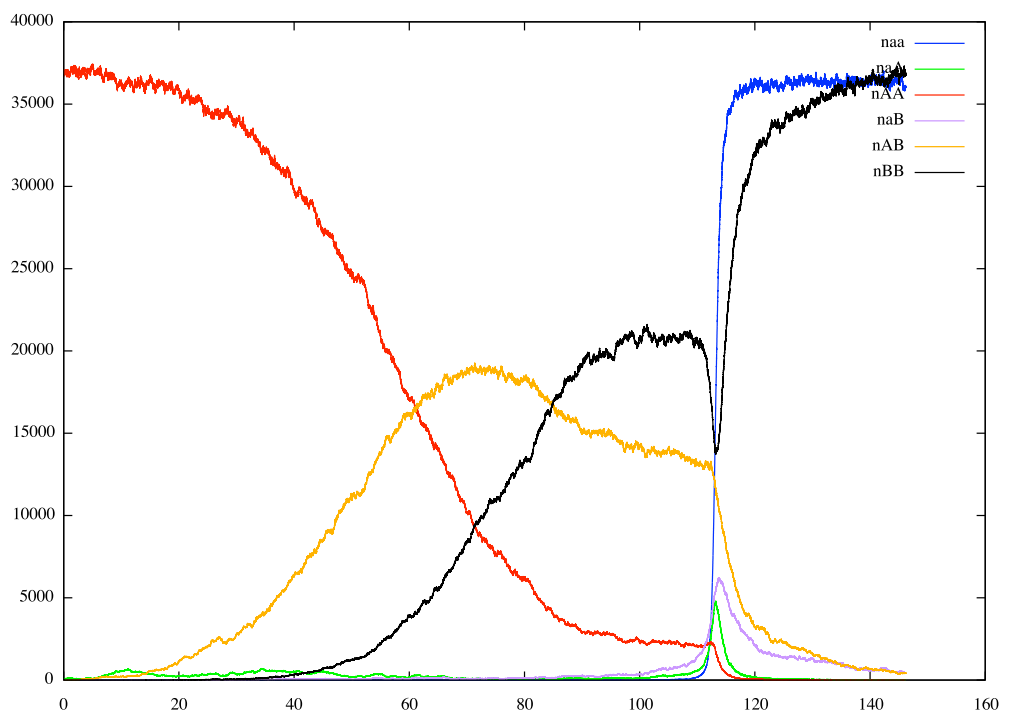

FIGURE 2. Simulation of the stochastic system for $f=6, D=0.7, \Delta=$ $0.1, c=1, \eta=0.02, \varepsilon=0.014$ and $K=7000$.

\section{Model SETUP}

Let $\mathcal{G}=\{a a, a A, A A, a B, A B, B B\}$ be the genotype space. Let $n_{i}(t)$ be the number of individuals with genotype $i \in \mathcal{G}$ in the population at time $t$ and set $n_{i}^{K}(t) \equiv \frac{1}{K} n_{i}(t)$.

Definition 2.1. The equilibrium size of a monomorphic $u u$-population, $u \in\{a, A, B\}$, is the fixed point of a 1-dimensional Lotka-Volterra equation and is given by

$$
\bar{n}_{u}=\frac{f_{u u}-D_{u u}}{c_{u u, u u}} .
$$

Definition 2.2. For $u, v \in\{a, A, B\}$, we call

$$
S_{u v, u u}=f_{u v}-D_{u v}-c_{u v, u u} \bar{n}_{u}
$$

the invasion fitness of a mutant $u v$ in a resident $u u$-population.

We take the phenotypic viewpoint and assume that the $B$-allele is the most dominant one. That means the ascending order of dominance (in the Mendelian sense) is given by $a<A<B$, i.e.

(1) phenotype $a$ consists of the genotype $a a$,

(2) phenotype $A$ consists of the genotypes $a A, A A$,

(3) phenotype $B$ consists of the genotypes $a B, A B, B B$.

For simplicity, we assume that the fertilities are the same for all genotypes, and that natural death rates are the same within the three different phenotypes. Moreover, we assume that there can be no reproduction between $a$ and $B$ phenotypes.

To sumarize, we make the following Assumptions $(\mathbf{B})$ on the rates:

(B1) Fertilities. For all $i \in \mathcal{G}$, and some $f>0$

$$
f_{i} \equiv f
$$


(B2) Natural death rates. The difference in fitness of the three phenotypes is realised by choosing a slightly higher natural death-rate of the $a$-phenotype and a slightly lower death-rate for the $B$-phenotype. For some $0<\Delta<D$,

$$
\begin{aligned}
D_{a a} & =D+\Delta, \\
D_{A A} \equiv D_{a A} & =D \\
D_{a B} \equiv D_{A B} \equiv D_{B B} & =D-\Delta
\end{aligned}
$$

(B3) Competition rates. We require that phenotypes $a$ and $B$ do not compete with each other. Moreover, we introduce a parameter $\eta \geq 0$ which lowers the competition between $B B$ and $a A$. For some $0 \leq \eta<c$,

$$
\left(c_{i, j}\right)_{\{i, j\} \in \mathcal{G} \times \mathcal{G}}=\begin{array}{c|c|cc|cccc} 
& a a & a A & A A & a B & A B & B B \\
\hline a a & c & c & c & 0 & 0 & 0 \\
\hline a A & c & c & c & c & c & c-\eta \\
A A & c & c & c & & c & c & c \\
\hline a B & 0 & c & c & c & c & c \\
A B & 0 & c & c & c & c & c \\
B B & 0 & c-\eta & c & c & c & c \\
\hline
\end{array}
$$

A biological interpretation for this kind of competition could be that it is coded in the alleles which food an individual with a given genotype prefers. Since an $A B$-individual shares one $B$-allele with a $B B$-individual, they compete stronger for the same food than $A A$ with $B B$ since those have completely different alleles.

(B4) Reproductive compatibility. We require that phenotypes $a$ and $B$ do not reproduce with each other.

$\left(R_{i}(j)\right)_{\{i, j\} \in \mathcal{G} \times \mathcal{G}} \equiv$\begin{tabular}{c|c|cc|ccc} 
& $a a$ & $a A$ & $A A$ & $a B$ & $A B$ & $B B$ \\
\hline$a a$ & 1 & 1 & 1 & 0 & 0 & 0 \\
\hline$a A$ & 1 & 1 & 1 & 1 & 1 & 1 \\
$A A$ & 1 & 1 & 1 & 1 & 1 & 1 \\
\hline$a B$ & 0 & 1 & 1 & 1 & 1 & 1 \\
$A B$ & 0 & 1 & 1 & 1 & 1 & 1 \\
$B B$ & 0 & 1 & 1 & 1 & 1 & 1 \\
\hline
\end{tabular}

Observe that, under Assumptions (B),

$$
\begin{aligned}
S_{A B, A A} & =f-(D-\Delta)-c \bar{n}_{A A}=f-D+\Delta-c \frac{f-D}{c}=\Delta, \\
S_{a a, B B} & =f-D-\Delta .
\end{aligned}
$$

Therefore, the mutant $A B$ has a positive invasion fitness in the population $A A$, as well as $a a$ in the $B B$ population (due to the absence of competition between them).

2.1. Birth rates. We assume that there is no recombination between phenotypes $a$ and $B$. Thus,

(1) the pool of possible partners for the phenotype $a$ consists of phenotypes $a$ and $A$; the total population of this pool is denoted by

$$
\Sigma_{3}:=n_{a a}+n_{a A}+n_{A A},
$$


(2) the pool of possible partners for the phenotype $A$ consists of the three phenotypes $a, A$, and $B$; the total population of this pool is denoted by

$$
\Sigma_{6}:=n_{a a}+n_{a A}+n_{A A}+n_{a B}+n_{A B}+n_{B B},
$$

(3) the pool of possible partners for the phenotype $B$ consists of phenotypes $A$ and $B$; the total population of this pool is denoted by

$$
\Sigma_{5}:=n_{a A}+n_{A A}+n_{a B}+n_{A B}+n_{B B} .
$$

Computing the reproduction rates with the Mendelian rules as described in (1.5) leads to the following (time-dependant) birth-rates $b_{i}=b_{i}(n(t))$ :

$$
\begin{aligned}
b_{a a}= & f \frac{n_{a a}\left(n_{a a}+\frac{1}{2} n_{a A}\right)}{n_{a a}+n_{a A}+n_{A A}}+f \frac{\frac{1}{2} n_{a B}\left(\frac{1}{2} n_{a A}+\frac{1}{2} n_{a B}\right)}{n_{a A}+n_{A A}+n_{a B}+n_{A B}+n_{B B}} \\
& +f \frac{\frac{1}{2} n_{a A}\left(n_{a a}+\frac{1}{2} n_{a A}+\frac{1}{2} n_{a B}\right)}{n_{a a}+n_{a A}+n_{A A}+n_{a B}+n_{A B}+n_{B B}} \\
b_{a A}= & f \frac{n_{a a}\left(\frac{1}{2} n_{a A}+n_{A A}\right)}{n_{a a}+n_{a A}+n_{A A}}+f \frac{\frac{1}{2} n_{a A}\left(\frac{1}{2} n_{a B}+\frac{1}{2} n_{A B}\right)+\frac{1}{2} n_{a B}\left(n_{A A}+n_{A B}\right)}{n_{a A}+n_{A A}+n_{a B}+n_{A B}+n_{B B}} \\
& +f \frac{\left(\frac{1}{2} n_{a A}+n_{A A}\right)\left(n_{a a}+n_{a A}+\frac{1}{2} n_{a B}\right)+\frac{1}{4} n_{a A} n_{A B}}{n_{a a}+n_{a A}+n_{A A}+n_{a B}+n_{A B}+n_{B B}} \\
b_{A A}= & f \frac{\frac{1}{2} n_{A B}\left(\frac{1}{2} n_{a A}+n_{A A}+\frac{1}{2} n_{A B}\right)}{n_{a A}+n_{A A}+n_{a B}+n_{A B}+n_{B B}}+f \frac{\left(\frac{1}{2} n_{a A}+n_{A A}\right)\left(\frac{1}{2} n_{a A}+n_{A A}+\frac{1}{2} n_{A B}\right)}{n_{a a}+n_{a A}+n_{A A}+n_{a B}+n_{A B}+n_{B B}} \\
b_{a B}= & f \frac{\left(\frac{1}{2} n_{a A}+n_{a B}\right)\left(\frac{1}{2} n_{a B}+\frac{1}{2} n_{A B}+n_{B B}\right)}{n_{a A}+n_{A A}+n_{a B}+n_{A B}+n_{B B}}+f \frac{\frac{1}{2} n_{a A}\left(\frac{1}{2} n_{a B}+\frac{1}{2} n_{A B}+n_{B B}\right)}{n_{a a}+n_{a A}+n_{A A}+n_{a B}+n_{A B}+n_{B B}} \\
b_{B B}= & f \frac{\frac{1}{4}\left(n_{a B}+n_{A B}+2 n_{B B}\right)^{2}}{n_{a A}+n_{A A}+n_{a B}+n_{A B}+n_{B B}} . \\
b_{A B}= & f \frac{\left(\frac{1}{2} n_{a A}+n_{A A}+n_{A B}\right)\left(\frac{1}{2} n_{a B}+\frac{1}{2} n_{A B}+n_{B B}\right)}{n_{a A}+n_{A A}+n_{a B}+n_{A B}+n_{B B}}+f \frac{\left(\frac{1}{2} n_{a A}+n_{A A}\right)\left(\frac{1}{2} n_{a B}+\frac{1}{2} n_{A B}+n_{B B}\right)}{n_{a a}+n_{a A}+n_{A A}+n_{a B}+n_{A B}+n_{B B}},
\end{aligned}
$$

2.2. Death rates. The death rates are the sum of the natural death and the competition:

$$
\begin{aligned}
d_{a a} & =n_{a a}\left(D+\Delta+c\left(n_{a a}+n_{a A}+n_{A A}\right)\right), \\
d_{a A} & =n_{a A}\left(D+c\left(n_{a a}+n_{a A}+n_{A A}+n_{a B}+n_{A B}\right)+(c-\eta) n_{B B}\right), \\
d_{A A} & =n_{A A}\left(D+c\left(n_{a a}+n_{a A}+n_{A A}+n_{a B}+n_{A B}+n_{B B}\right)\right), \\
d_{a B} & =n_{a B}\left(D-\Delta+c\left(n_{a A}+n_{A A}+n_{a B}+n_{A B}+n_{B B}\right)\right), \\
d_{A B} & =n_{A B}\left(D-\Delta+c\left(n_{a A}+n_{A A}+n_{a B}+n_{A B}+n_{B B}\right)\right), \\
d_{B B} & =n_{B B}\left(D-\Delta+(c-\eta) n_{a A}+c\left(n_{A A}+n_{a B}+n_{A B}+n_{B B}\right)\right) .
\end{aligned}
$$


2.3. Large population limit. By [15] or [7], for large populations, the behaviour of the stochastic process is close to the solution of a deterministic equation.

Proposition 2.3 (Generalisation of Proposition 3.2 in [7]).

Let $T>0$ and $C \subset \mathbb{R}_{+}^{6}$ be a compact set. Assume that the initial condition $n^{K}(0)=$ $\frac{1}{K}\left(n_{a a}(0), n_{a A}(0), n_{A A}(0), n_{a B}(0), n_{A B}(0), n_{B B}(0)\right)$ converges almost surely to a deterministic vector $x^{0}=\left(x_{1}^{0}, x_{2}^{0}, x_{3}^{0}, x_{4}^{0}, x_{5}^{0}, x_{6}^{0}\right) \in C$, as $K \rightarrow \infty$.

Let $\tilde{n}\left(t, x^{0}\right)$ denote the solution to

$$
\begin{aligned}
\dot{n}(t) & =b(n(t))-d(n(t)) \equiv F(n(t)), \\
r m \text { i.e. } \quad \dot{n}_{i}(t) & =b_{i}(n(t))-\left(D_{i}+\sum_{j \in \mathcal{G}} c_{i, j} n_{j}(t)\right) n_{i}(t), \quad \text { for all } i \in \mathcal{G}
\end{aligned}
$$

with initial condition $x^{0}$, where $\left(b_{i}\right)_{i \in \mathcal{G}}$ and $\left(d_{i}\right)_{i \in \mathcal{G}}$ are given in (2.12)-(2.17) and (2.18)(2.23). Then, for all $T>0$,

$$
\left.\lim _{K \rightarrow \infty} \sup _{t \in[0, T]} \mid n_{i}^{K}(t)-\tilde{n}_{i}\left(t, x^{0}\right)\right) \mid=0, \quad \text { a.s. }
$$

for all $i \in \mathcal{G}$.

2.4. Initial condition. Fix $\varepsilon>0$ sufficiently small. For the results below, we will consider the dynamical system (2.24) starting with the initial condition:

$$
\begin{aligned}
\bar{n}_{A} \geq n_{A A}(0) & \geq \bar{n}_{A}-\Theta(\varepsilon), \\
n_{a A}(0) & =\varepsilon \\
n_{a a}(0) & =\Theta\left(\varepsilon^{2}\right), \\
n_{A B}(0) & =\varepsilon^{3} \\
n_{B B}(0) & =0 \\
n_{a B}(0) & =0
\end{aligned}
$$

Remark. In all the figures below, the choice of parameters is the following:

$$
f=6, \quad D=0.7, \quad \Delta=0.1, \quad c=1, \quad \varepsilon=0.01,
$$

and the parameter $\eta$ is specified on each picture.

\section{RESUlts}

We are working with a 6-dimensional dynamical system, and computing all the fixed points analytically is impossible for a general choice of the parameters. We can however compute those which are relevant for our study. We will call $p_{A}$ (resp. $p_{B}$ ) the fixed points corresponding to the monomorphic $A A$ (resp. $B B$ ) population at equilibrium, and $p_{a B}$ the fixed point corresponding to the coexisting $a a$ and $B B$ populations. Setting the relevant populations to 0 and solving $\dot{n}(t)=0$, we get:

$$
\begin{aligned}
p_{A} & =\left(0,0, \bar{n}_{A}, 0,0,0\right) \\
p_{B} & =\left(0,0,0,0,0, \bar{n}_{B}\right) \\
p_{a B} & =\left(\bar{n}_{a}, 0,0,0,0, \bar{n}_{B}\right)
\end{aligned}
$$

where $\bar{n}_{a}=\frac{f-D-\Delta}{c}, \bar{n}_{A}=\frac{f-D}{c}$, and $\bar{n}_{B}=\frac{f-D+\Delta}{c}$. Note that the $B B$ equilibrium population is the same in $p_{B}$ and $p_{a B}$. This is due to the non-interaction between phenotypes $a$ and $B$.

Our general result is that starting with initial conditions (2.27)-(2.32), that is close to $p_{A}$ (with small coordinates in directions $a a, a A$ and $A B$ ), and under minimal assumptions 

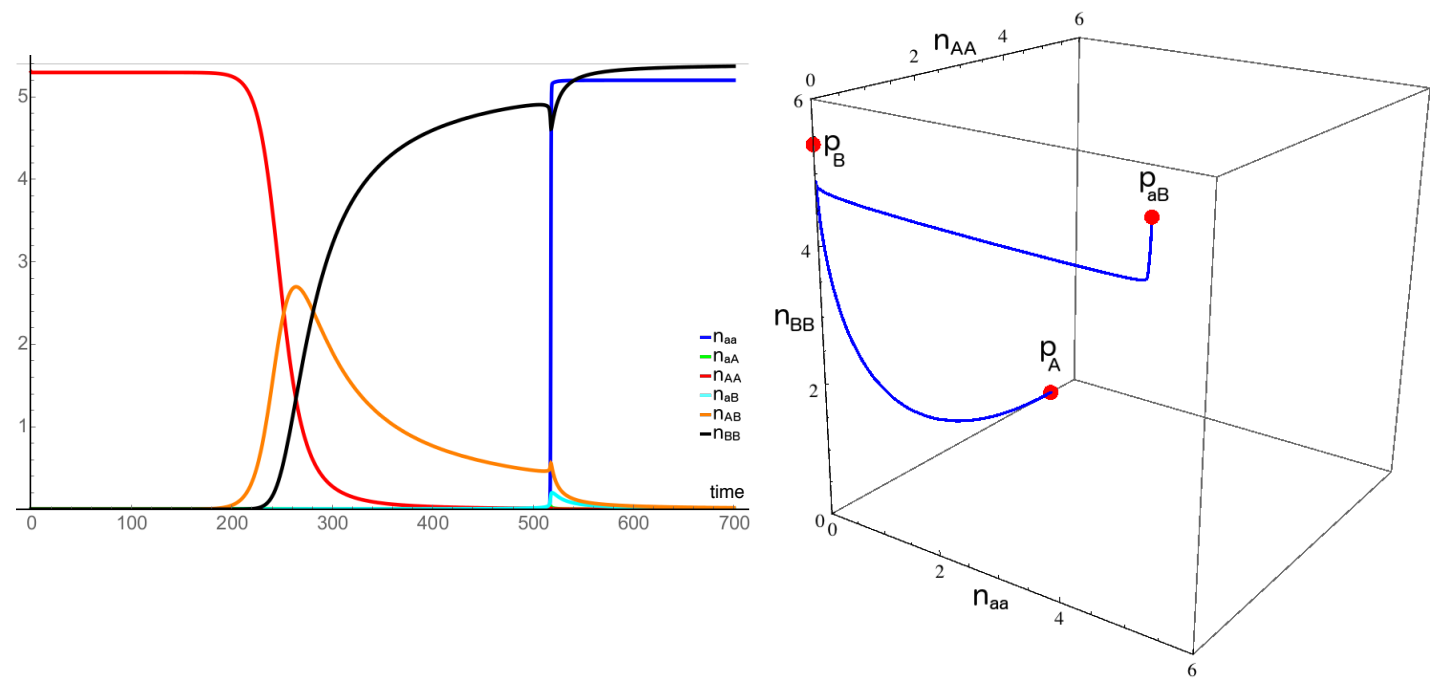

FIGURE 3. General qualitative behaviour of $\left\{n_{i}(t), i \in \mathcal{G}\right\}$ and projection of the dynamical system on the coordinates $a a, A A$ and $B B$. The re-invasion of the $a a$ population happens sooner and sooner as $\eta$ grows $(\eta=0.02$ for both pictures).

on the parameters, the system gets very close to $p_{B}$ before finally converging to $p_{a B}$, see Figure 3 .

Theorem 3.1. Consider the dynamical system (2.24) started with initial conditions (2.27)(2.32). Suppose the following Assumptions $(\boldsymbol{C})$ on the parameters hold:

(C1) $\Delta$ sufficiently small,

(C2) $f$ sufficiently large,

(C3) $0 \leq \eta<c / 2$.

Then the system converges to the fixed point $p_{a B}$. More precisely, for any fixed $\delta>0$, as $\varepsilon \rightarrow 0$, it reaches a $\delta$-neighbourhood of $p_{a B}$ in a time of order $\Theta\left(\varepsilon^{-1 /\left(1+\eta \bar{n}_{B}-\Delta\right)}\right)$.

Moreover, it holds:

(1) for $\eta=0$, the amount of allele $a$ in the population decays to $\Theta\left(\varepsilon^{1+\Delta /(1+\Delta)}\right)$ before reaching $\Theta(1)$,

(2) for $\eta>\frac{4 \Delta}{\bar{n}_{B}}$, the amount of a allele in the population is bounded below by $\Theta(\varepsilon)$ for all $t>0$.

Remark. For $\eta$ large, we prove that the fixed point $p_{a B}$ is unstable. We observe numerically that the system is attracted to a fixed point where all the 6 populations coexist, but we do not prove this.

Let us now briefly discuss the linear stability of the relevant fixed points and give an heuristics of the proof of Theorem 3.1 .

3.1. Linear stability analysis. The Jacobian matrix $J_{F}:=\left(\partial F_{i} / \partial n_{j}\right)_{i j}$ of the map $F$ defined in (2.24) can be explicitly computed at $p_{A}$ and $p_{a B}$ and the situation is as follows:

- The eigenvalues of $J_{F}\left(p_{A}\right)$ are $0, \Delta>0$ and $-(f-D),-(f+\Delta),-(f-\Delta)$ (double) which are all strictly negative under Assumptions (C). The fixed point $p_{A}$ is thus unstable. 
- The eigenvalues of $J_{F}\left(p_{a B}\right)$ are 0 (double), and - $(2 f-D),-(f-D+\Delta),-(f-D-$ $\Delta),-((f-D)(5 f-4 D)+f \Delta) /\left(4(f-D)+\eta \bar{n}_{B}\right)$ which are strictly negative under Assumptions (C). The linear analysis thus does not imply the stability of $p_{a B}$ but the Phase 4 of our proof will (see Section 4.5) .

It turns out that $J_{F}\left(p_{B}\right)$ is singular but as the invasion fitness of $a a$ is positive, i.e. $S_{a a, B B}>0$ (see (2.7)), this implies that a small perturbation in the first coordinate will be amplified, and thus implies the instability of the fixed point $p_{B}$.

3.2. Heuristics of the proof. Recall we start the dynamical system (2.24) with initial conditions (2.27)-(2.32). A numerical solution of the system is provided on Figure 4.

Remark. Assumption $\mathrm{C} 1$ of Theorem 3.1 is needed throughout the proof in order to be able to use the results of [25] which rely on the Center Manifold Theorem (a line of fixed points becomes an invariant line under small enough perturbation).

Phase 1. Time period: until $n_{A B}=\varepsilon_{0}$.

The mutant population, consisting of all individuals of phenotype $B$, first grows up to $\varepsilon_{0}$ exponentially fast with rate $\Delta$ without perturbing the behaviour of the 3 -system $(a a, a A, A A)$. The rate of growth corresponds to the invasion fitness of $A B$ in the resident population $A A$, see (2.7). Following [25], $A A$ stays close to $\bar{n}_{A}$, while $a A$ and $a a$ continue to decay like $1 / t$ and $1 / t^{2}$ respectively. The duration $T_{1}$ of this phase is such that $\Theta\left(\varepsilon^{3}\right) e^{t \Delta}=\Theta(1) \Leftrightarrow T_{1}=\Theta(|\log \varepsilon|)$.

Phase 2. Time period: until $n_{a A}=\Theta\left(n_{A A}\right)$.

The evolution is a perturbation of an effective 3 -system $(A A, A B, B B)$ which behaves exactly the same as in [25], since the parameters satisfy the same hypotheses (slightly lower death rate for phenotype $B$ than for phenotype $A$, and constant competition parameters). A comparison result (following Theorem 4.5 below) shows that this 3-system is almost unperturbed until $n_{a A}=\Theta\left(n_{A A}\right)$. If that happens in a time $T_{2}$ diverging with $\varepsilon$ (which we ensure throughout the calculation), we thus know that $B B$ approaches $\bar{n}_{B}$, while $n_{A B} \propto 1 / t$ and $n_{A A} \propto 1 / t^{2}$.

The important fact in this phase is that the amount of allele $a$ in the population decays for $\eta$ small while it increases for large enough $\eta$. Indeed, let us derive some bounds on $\Sigma_{a A, a B}=n_{a A}+n_{a B}$. The population $\Sigma_{a A, a B}$ reproduces by taking the dominant allele in a population of order $\Theta(1)$ and the allele $a$ in itself. Thus its birth rate satisfy $b_{\Sigma_{a A, a B}} \approx f \Sigma_{a A, a B}$. We can compute its death rate exactly and use that $n_{B B} \approx \Sigma_{5} \approx \bar{n}_{B}$ :

$$
\begin{aligned}
d_{\Sigma_{a A, a B}} & =\Sigma_{a A, a B}\left(D-\Delta+c \Sigma_{5}\right)-\eta n_{a A} n_{B B}+\Delta n_{a A} \\
& \approx f \Sigma_{a A, a B}-n_{a A}\left(\eta \bar{n}_{B}-\Delta\right), \\
\dot{\Sigma}_{a A, a B} & \approx n_{a A}\left(\eta \bar{n}_{B}-\Delta\right) \\
& =\Theta\left(\Sigma_{a A, a B} \cdot n_{A B}\right)\left(\eta \bar{n}_{B}-\Delta\right)
\end{aligned}
$$

The last equality comes from the fact that $a A$ newborns have mainly their $a$ allele coming from $\Sigma_{a A, a B}$ and their $A$ allele coming from $A B$. Using the $1 / t$ decay of $A B$ we get:

$$
\dot{\Sigma}_{a A, a B} \approx \frac{\Theta\left(\Sigma_{a A, a B}\right)}{\Theta(1)+\Theta(1) t}\left(\eta \bar{n}_{B}-\Delta\right)
$$


As $\Sigma_{a A, a B}\left(T_{1}\right)=\Theta(\varepsilon)$ we deduce that $\Sigma_{a A, a B}(t)=\Theta(\varepsilon)(\Theta(1)+\Theta(1) t)^{\Theta\left(\eta \bar{n}_{B}-\Delta\right)}$, and thus $n_{a A}=\Theta\left(n_{A B} \cdot \Sigma_{a A, a B}\right)=\Theta(\varepsilon)(\Theta(1)+\Theta(1) t)^{\Theta\left(\eta \bar{n}_{B}-\Delta\right)} /(\Theta(1)+\Theta(1) t)$. By solving $n_{a A}=\Theta\left(n_{A A}\right)=\Theta\left(n_{A B}^{2}\right)$ we get the order of magnitude of $T_{2}=\Theta\left(\varepsilon^{-1 /\left(1+\eta \bar{n}_{B}-\Delta\right)}\right)$. Note that for $\eta=0, \Sigma_{a A, a B}\left(T_{2}\right)=\Theta\left(\varepsilon^{1+\Delta /(1-\Delta)}\right)$. Moreover, (3.7) implies that for $\eta>\Delta / \bar{n}_{B}$, we have $\dot{\Sigma}_{a A, a B}>0$, which proves points 1 and 2 of Theorem 3.1 .

Phase 3. Time period: until $a$ a reaches equilibrium.

The fact that $n_{a A}=\Theta\left(n_{A A}\right)$ has a crucial effect on the birth rate of $a a$ (see (2.12) $)$ since the term $\left(n_{a a}+\frac{1}{2} n_{a A}\right) /\left(n_{a a}+n_{a A}+n_{A A}\right)$ becomes of order $\Theta(1)$. As long as $A A$ stays smaller than $\Theta(\varepsilon)$, we get a lower bound on $n_{a a}$ which grows exponentially fast since $f$ is chosen large enough (Assumption $\mathrm{C} 2$ ):

$$
\begin{aligned}
& b_{a a} \geq f n_{a a} \Theta(1), \\
& d_{a a} \leq n_{a a}(D+\Delta+\Theta(\varepsilon)), \\
& \dot{n}_{a a} \geq n_{a a}(f \Theta(1)-D-\Delta-\Theta(\varepsilon)) .
\end{aligned}
$$

As $a a$ grows, it makes $\Sigma_{a A, a B}$ grow, and thus $A A$ and $A B$ as well. We have to show that this could not prevent $a a$ from reaching equilibrium. We do not give a detailed argument here, but essentially, the presence of the macroscopic $B B$ population prevents all the non- $a a$ populations to grow too much. Note that if $\eta$ is too large, then $a A$ could get a positive fitness and grow to a macroscopic level. That is why we have to impose Assumption C3, which will become clearer heuristically in the next phase. We recall that $a a$ does not compete with $B B$ and thus it grows exponentially fast with rate $f-(D+\Delta)$ until an $\varepsilon_{0}$-neighbourhood of the fixed point where $a a$ and $B B$ coexist. The rate of growth corresponds to the invasion fitness of $a a$ in the resident population $B B$, see 2.7). Note that, due to Assumption $C 2$, this rate is much larger than the invasion rate of $B B$ into $A A$. That is why the fourth phase looks very steep on Figure 4, see the stretched version on Figure 6. This phase lasts a time $T_{3}=\Theta(|\log \varepsilon|)$.

Phase 4. The Jacobian matrix of the field (2.24) at the fixed point $p_{a B}$ has two zero, and 4 negative eigenvalues. $p_{a B}$ is thus a non-hyperbolic equilibrium point of the system and linearisation fails to determine its stability properties. Instead, we use the result of center manifold theory ( [18,26]) that asserts that the qualitative behaviour of the dynamical system in a neighbourhood of the non-hyperbolic critical point $p_{a B}$ is determined by its behaviour on the center manifold near $p_{a B}$. Using the Center Manifold Theorem, we show that asymptotically as $f \rightarrow \infty$, the field is attractive for $\eta<c \cdot r_{\max }$ where $r_{\max } \simeq 0.593644$ is the maximum of the rational function (4.334). Thus $p_{a B}$ is a stable fixed point which is approached with speed $\frac{1}{t}$ as long as $\eta<c \cdot r_{\max }$. For higher values of $\eta$, numerical solutions show that the system converges to a fixed point where the 6 populations co-exist, but we do not prove this.

\section{PROOF}

Definition 4.1. Let $x, y, z \in\{a a, a A, A A, a B, A B, B B\}$ and $h \in \mathbb{R}$. We define

$$
\begin{aligned}
T^{x=y} & =\inf \left\{t>0: n_{x}(t)=n_{y}(t)\right\}, \\
T^{x=\delta y} & =\inf \left\{t>0: n_{x}(t)=\delta n_{y}(t)\right\},
\end{aligned}
$$




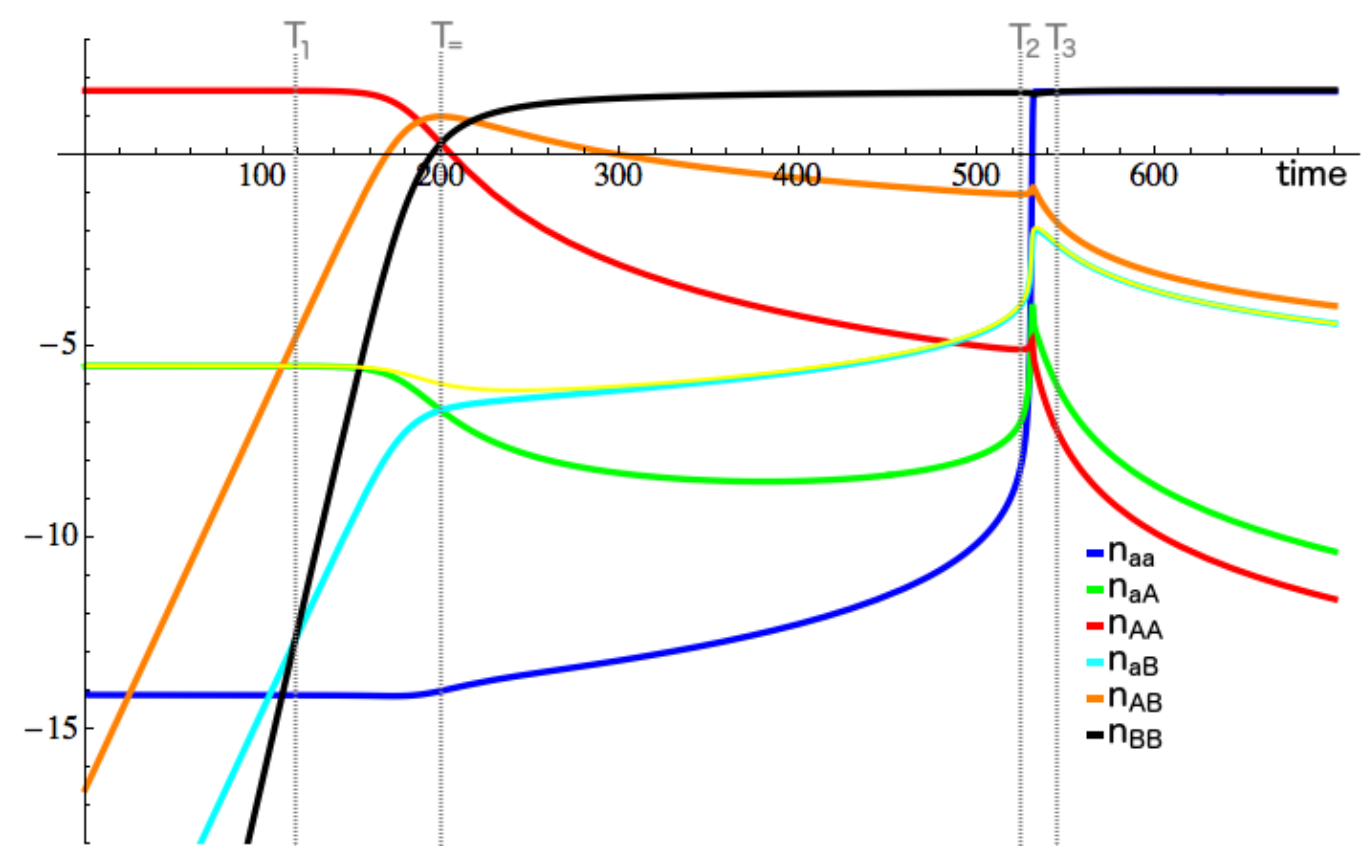

FIGURE 4. Numerical solution of the deterministic system for $\eta=0.02$, logplot.

$$
\begin{aligned}
T_{h}^{x} & =\inf \left\{t>0: n_{x}(t)>h\right\} \\
T_{h}^{x+y} & =\inf \left\{t>0: n_{x}(t)+n_{y}(t)>h\right\} \\
T_{h}^{x+y+z} & =\inf \left\{t>0: n_{x}(t)+n_{y}(t)+n_{z}(t)>h\right\}
\end{aligned}
$$

Moreover, let

$$
\Delta>\varepsilon_{0}>\varepsilon>0 .
$$

The value $\varepsilon_{0}$ is the small order 1 level in the Phase 1, see the proof heuristics (Section 3.2). We consider $\Delta$ fixed and sufficiently small, and will first send $\varepsilon \rightarrow 0$ and then $\varepsilon_{0} \rightarrow 0$.

4.1. Preliminaries. We first prove general facts which will be useful through the proof.

Lemma 4.2. Let $c>0$ and $n(t)$ be such that

- $\dot{n}(t) \leq g(t)-c \cdot n(t)$ for all $t \in \mathcal{T} \subset \mathbb{R}^{+}$,

- $c \cdot n(0) \leq g(0)$,

if $c \cdot n(t)=g(t) \Rightarrow c \cdot \dot{n}(t) \leq \dot{g}(t)$ for all $t \in \mathcal{T}$ then $c \cdot n(t) \leq g(t)$ for all $t \in \mathcal{T}$,

Proof. This is an easy analysis exercise.

Proposition 4.3. If $n_{a B}(0)<n_{A B}(0)$ then $n_{a B}(t) \leq n_{A B}(t)$.

Proof. Intuitively this inequality comes from the fact that phenotype $a$ individuals cannot reproduce with phenotype $B$. Indeed, if we consider the couples that could give rise to an $A B$ (resp. $a B)$ individual, they are of the form $\left(A g_{1}, B g_{2}\right)$ (resp. $\left.\left(a g_{1}, B g_{2}\right)\right)$, with $g_{1}, g_{2} \in$ $\{a, A, B\}$ and the combination $\left(A A, B g_{2}\right)$ is possible whereas $\left(a a, B g_{2}\right)$ is impossible. Here is the rigorous derivation of the result: We compare the birth- and the death-rates of $n_{A B}$ and $n_{a B}$

$$
\frac{d_{a B}}{n_{a B}}=D-\Delta+c\left(n_{a A}+n_{A A}+n_{a B}+n_{A B}+n_{B B}\right)=\frac{d_{A B}}{n_{A B}}
$$




$$
\begin{aligned}
& b_{a B}=f n_{a B} \frac{\frac{1}{2} n_{a B}+\frac{1}{2} n_{A B}+n_{B B}}{n_{a A}+n_{A A}+n_{a B}+n_{A B}+n_{B B}}+I_{a B}, \\
& b_{A B}=f n_{A B} \frac{\frac{1}{2} n_{a B}+\frac{1}{2} n_{A B}+n_{B B}}{n_{a A}+n_{A A}+n_{a B}+n_{A B}+n_{B B}}+I_{A B} .
\end{aligned}
$$

We see that the death-rates of the two populations are the same, whereas the birth-rates differ only in a factor which comes from the reproduction of the other populations. If we take a closer look to these factors $I_{a B}, I_{A B}$ under the assumption that $n_{a B}=n_{A B}$ we see that

$$
\begin{aligned}
I_{A B} & =f\left(\frac{1}{2} n_{a A}+n_{A A}\right)\left(\frac{\frac{1}{2} n_{a B}+\frac{1}{2} n_{A B}+n_{B B}}{n_{a A}+n_{A A}+n_{a B}+n_{A B}+n_{B B}}+\frac{\frac{1}{2} n_{a B}+\frac{1}{2} n_{A B}+n_{B B}}{n_{a a}+n_{a A}+n_{A A}+n_{a B}+n_{A B}+n_{B B}}\right) \\
& =I_{a B}+f n_{A A}\left(\frac{\frac{1}{2} n_{a B}+\frac{1}{2} n_{A B}+n_{B B}}{n_{a A}+n_{A A}+n_{a B}+n_{A B}+n_{B B}}+\frac{\frac{1}{2} n_{a B}+\frac{1}{2} n_{A B}+n_{B B}}{n_{a a}+n_{a A}+n_{A A}+n_{a B}+n_{A B}+n_{B B}}\right) .
\end{aligned}
$$

Thus $I_{A B}>I_{a B}$. Hence, $\dot{n}_{A B}>\dot{n}_{a B}$ and $n_{A B}(t)$ stays above $n_{a B}(t)$ for all $t>0$.

\subsection{Phase 1: Perturbation of the 3-system $(a a, a A, A A)$ until $A B$ reaches $\Theta(1)$.}

We start with initial conditions given by 2.27)-2.32. We will show that the mutant population, consisting of all individuals of phenotype $B$, grows up to some $\varepsilon_{0}>\varepsilon$ without perturbing the behaviour of the 3 -system $(a a, a A, A A)$ in this time. Let

$$
T_{1}:=T_{\varepsilon_{0}}^{a B+A B+B B} .
$$

Proposition 4.4. With the initial conditions 2.27)-(2.32), for all $t \in\left[0, T_{1}\right]$, it holds,

(1) $n_{a B}(t) \leq \Theta\left(\varepsilon \varepsilon_{0}\right), n_{a A}(t) \leq \Theta(\varepsilon), n_{a a}(t) \leq \Theta\left(\varepsilon^{2}\right)$ and $\bar{n}_{A}-\Theta\left(\varepsilon_{0}\right) \leq n_{A A}(t) \leq \bar{n}_{A}$.

(2) $n_{B B}(t)=\Theta\left(n_{A B}^{2}(t)\right)$.

(3) $n_{A B}(t)$ grows exponentially with rate $\Delta$. It reaches the level $\varepsilon_{0}$ in a time at most of $\operatorname{order} \Theta\left(\log \left(\left(\varepsilon_{0} / \varepsilon^{3}\right)^{\frac{1}{\Delta-\Theta\left(\varepsilon_{0}\right)}}\right)\right)$.

Proof. Until $T_{1}$ the perturbation of the dynamics of the 3 -system (aa, $\left.a A, A A\right)$ is at most of order $\varepsilon_{0}$. Thus we have $\bar{n}_{A}-\Theta\left(\varepsilon_{0}\right) \leq n_{A A}(t) \leq \bar{n}_{A}+\Theta\left(\varepsilon_{0}\right)$, as well as $n_{a a}, n_{a A} \leq \Theta\left(\varepsilon_{0}\right)$. With this rough bounds we will find finer bounds.

(1) The $\Delta$ reduced death rate of the mutant $A B$ gives it a positive fitness, and the growth is exponential until it reaches a macroscopic level. For an upper bound on the time $T_{\varepsilon_{0}}^{a B+A B+B B}$, we have to construct a minorising process for $n_{A B}$. Indeed, let us compare the birth and death rates:

$$
\begin{aligned}
& b_{A B} \geq \frac{1}{2} n_{A B} \frac{2 f n_{A A}}{n_{A A}+\Theta\left(\varepsilon_{0}\right)}=n_{A B}\left(f-\Theta\left(\varepsilon_{0}\right)\right), \\
& d_{A B} \leq n_{A B}\left(D-\Delta+c \bar{n}_{A}+\Theta\left(\varepsilon_{0}\right)\right)=n_{A B}\left(f-\Delta+\Theta\left(\varepsilon_{0}\right)\right) .
\end{aligned}
$$

Hence, we get for the minorising process

$$
\begin{aligned}
\dot{n}_{A B} & \geq n_{A B}\left(\Delta-\Theta\left(\varepsilon_{0}\right)\right), \\
n_{A B}(t) & \geq \varepsilon^{3} e^{\left(\Delta-\Theta\left(\varepsilon_{0}\right)\right) t},
\end{aligned}
$$



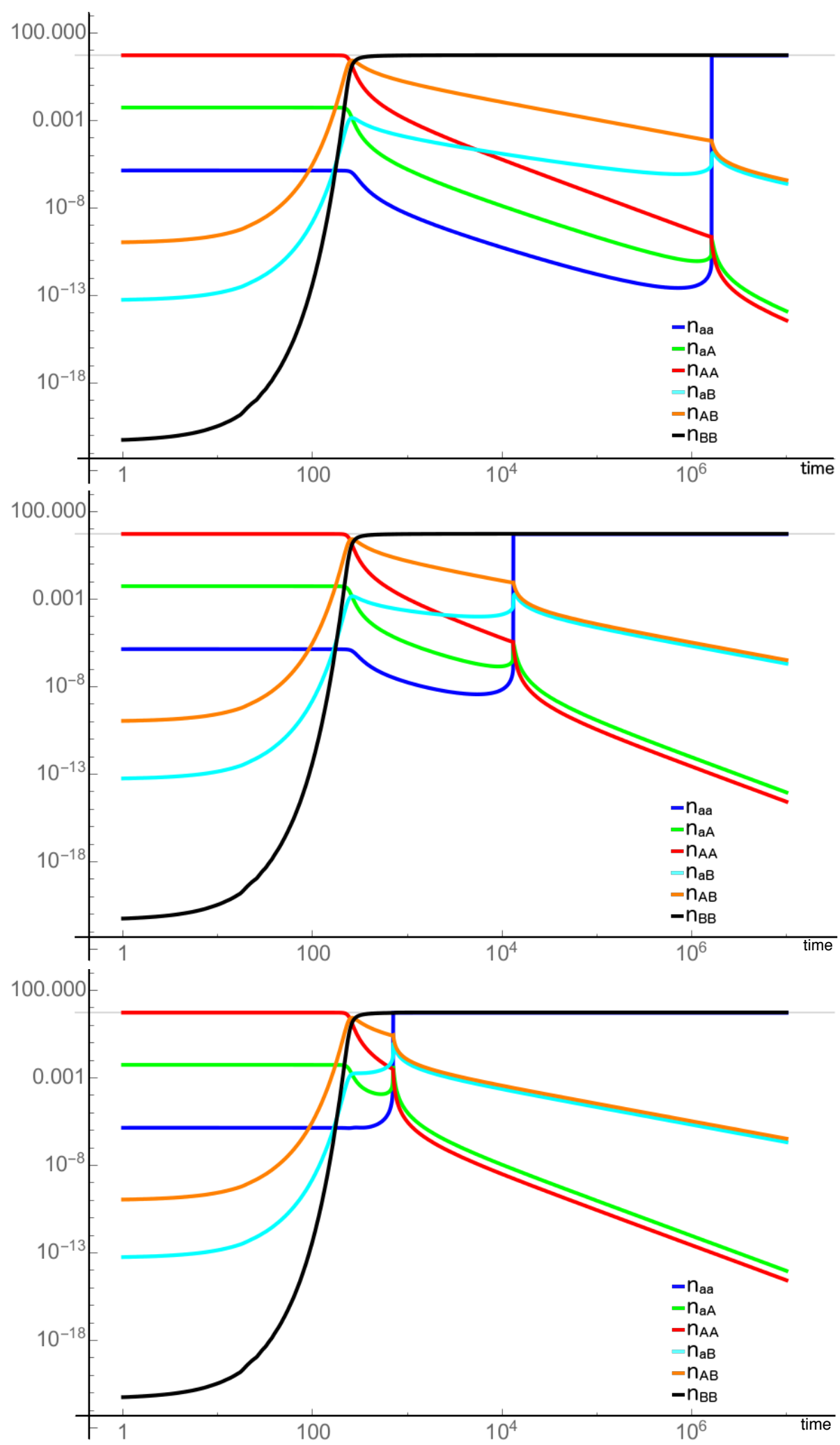

FIGURE 5. Log-plots of $\left\{n_{i}(t), i \in \mathcal{G}\right\}$ for $\eta=0$ (top), $\eta=0.003$ (center) and $\eta=0.014$ (bottom). 
and the time $T_{1}$ is at most of order $\Theta\left(\log \left(\left(\varepsilon_{0} / \varepsilon^{3}\right)^{\frac{1}{\Delta-\Theta\left(\varepsilon_{0}\right)}}\right)\right.$. For an lower bound on the time $T_{1}$, we have to construct a majorising process for $n_{A B}$. We compare the birth and death rates:

$$
\begin{aligned}
& b_{A B} \leq n_{A B}\left(f+\Theta\left(\varepsilon_{0}\right)\right), \\
& d_{A B} \geq n_{A B}\left(D-\Delta+c \bar{n}_{A}-\Theta\left(\varepsilon_{0}\right)\right)=n_{A B}\left(f-\Delta-\Theta\left(\varepsilon_{0}\right)\right) .
\end{aligned}
$$

Hence, we get for the majorising process

$$
\begin{array}{r}
\dot{n}_{A B} \leq n_{A B}\left(\Delta+\Theta\left(\varepsilon_{0}\right)\right), \\
n_{A B}(t) \leq \varepsilon^{3} e^{\left(\Delta+\Theta\left(\varepsilon_{0}\right)\right) t},
\end{array}
$$

and the time $T_{1}$ is at least of order $\Theta\left(\log \left(\left(\varepsilon_{0} / \varepsilon^{3}\right)^{\frac{1}{\Delta-\Theta\left(\varepsilon_{0}\right)}}\right)\right.$.

(2) Heuristically, the newborns of genotype $a A$ are still in majority produced by recombination of $A A$ and $a A$, because the mutant population is not large enough to contribute. The newborns of genotype $a B$ are in majority produced by reproduction of the $a A$-population with the $B$-population. Finally, the newborns of genotype $a a$ are in majority produced by recombination of $a A$ and $a A$, because the only mutant which could perturb it is $a B$ which is of smaller order.

(a) We show that $n_{a a} \leq n_{a A}^{2}$ or according to Lemma $4.2 \dot{n}_{a a}-2 \dot{n}_{a A} n_{a A} \leq 0$ when $n_{a a}=n_{a A}^{2}$.

Observe that $\dot{n}_{a a}-2 \dot{n}_{a A} n_{a A}=b_{a a}-2 n_{a A} b_{a A}-d_{a a}+2 n_{a A} d_{a A}$. The biggest contributing terms of $b_{a a}-2 n_{a A} b_{a A}$ and $d_{a a}-2 n_{a A} d_{a A}$ at $n_{a a}=n_{a A}^{2}$ are

$$
\begin{aligned}
& b_{a a}-2 n_{a A} b_{a A}=\frac{f}{4 \Sigma_{5}} n_{a B}^{2}-\frac{2 f}{\Sigma_{6}} n_{A A} n_{a A}^{2}, \\
& d_{a a}-2 n_{a A} d_{a A}=-n_{a A}^{2}\left(f-\Delta+\Theta\left(\varepsilon_{0}\right)\right) .
\end{aligned}
$$

Thus we get as long as $n_{a B}<n_{a A}$ :

$$
\begin{aligned}
\dot{n}_{a a}-2 \dot{n}_{a A} n_{a A} & =b_{a a}-2 n_{a A} b_{a A}-d_{a a}+2 n_{a A} d_{a A} \\
& \leq n_{a A}^{2}\left(f-\frac{2 f}{\Sigma_{6}} n_{A A}-\Delta+\Theta\left(\varepsilon_{0}\right)\right)+\frac{f}{4 \Sigma_{5}} n_{a B}^{2}<0 .
\end{aligned}
$$

(b) We show that $n_{a B}$ really stays smaller than $n_{a A}$, precisely we show that $n_{a B} \leq$ $n_{a A} n_{A B}$ or equivalently according to Lemma $4.2 \dot{n}_{a B}-\dot{n}_{a A} n_{A B}-\dot{n}_{A B} n_{a A} \leq 0$ at $n_{a B}=n_{a A} n_{A B}$.

The biggest contributing terms are

$$
\begin{aligned}
b_{a B}-n_{A B} b_{a A}-n_{a A} b_{A B}= & n_{a A} n_{A B}\left(\frac{f}{4 \Sigma_{5}}+\frac{f}{4 \Sigma_{6}}-\frac{f}{\Sigma_{6}} n_{A A}-\frac{f}{2 \Sigma_{5}} n_{A A}-\frac{f}{2 \Sigma_{6}} n_{A A}\right) \\
& +n_{a A} n_{B B}\left(\frac{f}{2 \Sigma_{5}}+\frac{f}{2 \Sigma_{6}}-\frac{f}{\Sigma_{5}} n_{A A}-\frac{f}{\Sigma_{6}} n_{A A}\right), \\
d_{a B}-n_{A B} d_{a A}-n_{a A} d_{A B}= & -n_{a A} n_{A B}\left(D+c \Sigma_{6}-\eta n_{B B}\right) .
\end{aligned}
$$

Thus we get

$$
\dot{n}_{a B}-\dot{n}_{a A} n_{A B}-\dot{n}_{A B} n_{a A} \leq n_{a A} n_{A B}\left(-f+\frac{f}{2 \Sigma_{5}}+\Theta\left(\varepsilon_{0}\right)\right)-n_{a A} n_{B B}\left(2 f-\frac{f}{\Sigma_{5}}-\Theta\left(\varepsilon_{0}\right)\right)<0 \text {. }
$$

(c) We show that $n_{a A} \leq \Theta\left(\varepsilon^{1-\varepsilon_{0}}\right)$.

We construct a majorising process on $a A$. The biggest contributing terms are

$$
\begin{aligned}
& b_{a A} \leq \frac{f}{\Sigma_{6}} n_{A A} n_{a A}+n_{a A} \Theta\left(\varepsilon_{0}\right), \\
& d_{a A} \geq n_{a A}\left(f-\Theta\left(\varepsilon_{0}\right)\right),
\end{aligned}
$$


and we get that

$$
\begin{aligned}
\dot{n}_{a A} & \leq \Theta\left(\varepsilon_{0}\right) n_{a A}, \\
n_{a A}(t) & \leq \varepsilon e^{\Theta\left(\varepsilon_{0}\right) t},
\end{aligned}
$$

what shows that until time $T_{1}, n_{a A} \leq \Theta\left(\varepsilon^{1-\varepsilon_{0}}\right)$.

(d) We show $\bar{n}_{A}-\varepsilon \leq \Sigma_{5} \leq \bar{n}_{A}+2 \Delta \varepsilon_{0}$.

We construct a minorising and a majorising processes on $\Sigma_{5}$ :

$$
\begin{aligned}
& b_{\Sigma_{5}} \leq f \Sigma_{5}+\Theta\left(n_{a a}\right), \\
& b_{\Sigma_{5}} \geq f \Sigma_{5}-\Theta\left(n_{a A}^{2}\right), \\
& d_{\Sigma_{5}} \geq \Sigma_{5}\left(D+c \Sigma_{5}\right)-\left(\Delta+2 \eta n_{a A}\right)\left(n_{a B}+n_{A B}+n_{B B}\right), \\
& d_{\Sigma_{5}} \leq \Sigma_{5}\left(D+c \Sigma_{5}+c n_{a a}\right), \\
& \dot{\Sigma}_{5} \leq \Sigma_{5}\left(f-D-c \Sigma_{5}\right)+\left(\Delta+2 \eta n_{a A}\right)\left(n_{a B}+n_{A B}+n_{B B}\right), \\
& \dot{\Sigma}_{5} \geq \Sigma_{5}\left(f-D-c \Sigma_{5}-c n_{a a}\right) .
\end{aligned}
$$

At the upper bound we have $\dot{\Sigma}_{5} \leq 0$ and at the lower bound $\dot{\Sigma}_{5} \geq 0$, which ensure the claimed bounds by Lemma 4.2

(3) The newborns of genotype $B B$ are in majority produced by recombination of $A B$ with itself. Indeed, by comparison of the birth- and death-rates,

$$
\begin{aligned}
b_{B B} & \leq f n_{B B} \frac{n_{a B}+n_{A B}+n_{B B}}{n_{a A}+n_{A A}+n_{a B}+n_{A B}+n_{B B}}+\frac{f}{n_{a A}+n_{A A}+n_{a B}+n_{A B}+n_{B B}} n_{A B}^{2} \\
& \leq f n_{B B} \Theta\left(\varepsilon_{0}\right)+\frac{f}{\bar{n}_{A}} n_{A B}^{2}+\Theta\left(\varepsilon_{0}^{3}\right), \\
b_{B B} & \geq f n_{B B} \Theta\left(\varepsilon_{0}\right)+\frac{f}{4 \bar{n}_{B}} n_{A B}^{2}, \\
d_{B B} & \geq n_{B B}\left(D-\Delta+c \bar{n}_{A}-\Theta\left(\varepsilon_{0}\right)\right)=n_{B B}\left(f-\Delta-\Theta\left(\varepsilon_{0}\right)\right), \\
d_{B B} & \leq n_{B B}\left(D-\Delta+c \bar{n}_{B}+\Theta\left(\varepsilon_{0}\right)\right)=n_{B B}\left(f+\Theta\left(\varepsilon_{0}\right)\right) .
\end{aligned}
$$

we get the upper bound for the process

$$
\dot{n}_{B B} \leq-n_{B B}\left(f\left(1-\Theta\left(\varepsilon_{0}\right)\right)-\Delta-\Theta\left(\varepsilon_{0}\right)\right)+\frac{f}{\bar{n}_{A}} n_{A B}^{2},
$$

and the lower bound

$$
\dot{n}_{B B} \geq-n_{B B}\left(f+\Theta\left(\varepsilon_{0}\right)\right)+\frac{f}{4 \bar{n}_{B}} n_{A B}^{2} .
$$

By applying Lemma 4.2 to $n=n_{B B}$ and $g=n_{A B}^{2}$ (with constants in front), as $n_{B B}(0)=0<n_{A B}(0)=\varepsilon^{3}$ and by Proposition 4.4 (2) $\dot{n}_{A B} \geq 0$ for all $t \in\left[0, T_{1}\right]$, we deduce that $n_{B B}(t) \leq \Theta\left(n_{A B}^{2}(t)\right)$ for all $t \in\left[0, T_{1}\right]$.

Note that Proposition 4.4 implies that $T_{1}=T_{\varepsilon_{0}}^{a B+A B+B B}=T_{\varepsilon_{0}}^{A B} \leq \Theta\left(\log \left(\left(\varepsilon_{0} / \varepsilon^{3}\right)^{\frac{1}{\Delta-\Theta\left(\varepsilon_{0}\right)}}\right)\right)$.

\subsection{Phase 2: Perturbation of the 3-system $(A A, A B, B B)$ until $n_{a A}=\Theta\left(n_{A A}\right)$.}

Let $\delta>0$ (to be chosen sufficiently small in the sequel). Let

$$
T_{2}:=T^{a A=\delta A A} \wedge T^{a B=\delta A B} \wedge T^{a a=a A \wedge a B}
$$

We will show that for $t \in\left[T_{1}, T_{2}\right]$ the system behaves as a main 3 -system $(A A, A B, B B)$ plus perturbations of order $\delta$. The 3 -system $(A A, A B, B B)$ behaves exactly the same as 
in [25] since the parameters satisfy the same hypotheses (slightly lower death rate for phenotype $B$ than for phenotype $A$ individuals, and constant competition parameters).

Moreover, the crucial role of the parameter $\eta$ is that the population containing an allele $a$ only continues to grow in this phase when $\eta$ is large enough. This is due to the smaller competition that $a A$ feels from $B B$, the $a A$ population is thus higher and induces the growth of $a B$.

We start by considering how the growth of $a A$ - and $a B$-populations can perturb the 3-system $(A A, A B, B B)$.

Lemma 4.5. Let $n^{u p}(t)$ be the population of the unperturbed 3-system $(A A, A B, B B)$. The 3-system ( $A A, A B, B B)$ satisfies

$$
\begin{aligned}
& \dot{n}_{B B} \geq \dot{n}_{B B}^{u p}-\left(n_{a A}+n_{a B}\right)\left(\frac{f\left(\frac{1}{2} n_{A B}+n_{B B}\right)^{2}}{\left(n_{A A}+n_{A B}+n_{B B}\right)^{2}}+c n_{B B}\right), \\
& \dot{n}_{B B} \leq \dot{n}_{B B}^{u p}+\left(n_{a A}+n_{a B}\right)\left(\frac{f\left(\frac{1}{4} n_{a B}+\frac{1}{2} n_{A B}+n_{B B}\right)}{\Sigma_{5}}+c n_{B B}\right), \\
& \dot{n}_{A B} \geq \dot{n}_{A B}^{u p}-\left(n_{a a}+n_{a A}+n_{a B}\right)\left(\frac{f\left(n_{A B}+n_{A A}\right)\left(\frac{1}{2} n_{A B}+n_{B B}\right)}{\left(n_{A A}+n_{A B}+n_{B B}\right)^{2}}+c n_{A B}\right), \\
& \dot{n}_{A B} \leq \dot{n}_{A B}^{u p}+\frac{f}{\Sigma_{5}} n_{a A}\left(\frac{1}{2} n_{a B}+\frac{1}{2} n_{A B}+n_{B B}\right)+\frac{f}{\Sigma_{5}} n_{a B}\left(\frac{1}{2} n_{A B}+n_{A A}\right), \\
& \dot{n}_{A A} \geq \dot{n}_{A A}^{u p}-\left(n_{a a}+n_{a A}+n_{a B}\right)\left(\frac{f\left(\frac{1}{2} n_{a A}+\frac{1}{2} n_{A B}+n_{A A}\right)^{2}}{\left(n_{A A}+n_{A B}+n_{B B}\right) 2}+c n_{A A}\right), \\
& \dot{n}_{A A} \leq \dot{n}_{A A}^{u p}+\frac{f}{2 \Sigma_{5}} n_{a A}\left(\frac{1}{2} n_{a A}+n_{A B}+n_{A A}\right) .
\end{aligned}
$$

Proof. We consider the rates of $A A, A B$ and $B B$ under the perturbation of $a a, a A$ and $a B$ :

$$
\begin{aligned}
b_{B B} & =\frac{f}{\Sigma_{5}}\left(\frac{1}{2} n_{A B}+n_{B B}\right)^{2}+\frac{f n_{a B}\left(\frac{1}{4} n_{a B}+\frac{1}{2} n_{A B}+n_{B B}\right)}{\Sigma_{5}} \\
& =b_{B B}^{u p}-\frac{f\left(\left(\frac{1}{2} n_{A B}+n_{B B}\right)^{2}\left(n_{a A}+n_{a B}\right)\right)}{\Sigma_{5}\left(n_{A A}+n_{A B}+n_{B B}\right)}+\frac{f n_{a B}\left(\frac{1}{4} n_{a B}+\frac{1}{2} n_{A B}+n_{B B}\right)}{\Sigma_{5}}, \\
d_{B B} & =d_{B B}^{u p}+c n_{B B}\left(n_{a B}+n_{a A}\right)-\eta n_{a A} n_{B B} .
\end{aligned}
$$

Thus,

$$
\begin{aligned}
& \dot{n}_{B B} \leq \dot{n}_{B B}^{u p}+\frac{f}{\Sigma_{5}} n_{a B}\left(\frac{1}{4} n_{a B}+\frac{1}{2} n_{A B}+n_{B B}\right)+\eta n_{a A} n_{B B}, \\
& \dot{n}_{B B} \geq \dot{n}_{B B}^{u p}-\frac{f\left(n_{a A}+n_{a B}\right)\left(\frac{1}{2} n_{A B}+n_{B B}\right)^{2}}{\Sigma_{5}\left(n_{A A}+n_{A B}+n_{B B}\right)}-c n_{B B}\left(n_{a A}+n_{a B}\right) .
\end{aligned}
$$

For the $A B$-population we get:

$$
\begin{aligned}
b_{A B}= & \frac{2 f\left(\frac{1}{2} n_{A B}+n_{B B}\right)\left(\frac{1}{2} n_{A B}+n_{A A}\right)}{\Sigma_{5}}-\frac{f n_{a a} n_{A A}\left(\frac{1}{2} n_{a B}+\frac{1}{2} n_{A B}+n_{B B}\right)}{\Sigma_{5} \Sigma_{6}}+\frac{f\left(n_{A A}+n_{A B}\right)}{2 \Sigma_{5}} n_{a B} \\
& +\frac{f n_{a B} n_{A A}}{2 \Sigma_{6}}+\frac{f n_{a A}\left(\frac{1}{2} n_{a B}+\frac{1}{2} n_{A B}+n_{B B}\right)}{2 \Sigma_{5}}+\frac{f n_{a A}\left(\frac{1}{2} n_{a B}+\frac{1}{2} n_{A B}+n_{B B}\right)}{2 \Sigma_{6}} \\
= & b_{A B}^{u p}+n_{a A}\left(\frac{1}{2} n_{a B}+\frac{1}{2} n_{A B}+n_{B B}\right)\left(\frac{f}{2 \Sigma_{5}}+\frac{f}{2 \Sigma_{6}}\right)+n_{a B} n_{A A}\left(\frac{f}{2 \Sigma_{5}}+\frac{f}{2 \Sigma_{6}}\right)+\frac{f n_{a B} n_{A B}}{2 \Sigma_{5}}
\end{aligned}
$$




$$
-\frac{f n_{a a} n_{A A}\left(\frac{1}{2} n_{a B}+\frac{1}{2} n_{A B}+n_{B B}\right)}{\Sigma_{5} \Sigma_{6}}-\frac{2 f\left(\frac{1}{2} n_{A B}+n_{A A}\right)\left(\frac{1}{2} n_{A B}+n_{B B}\right)\left(n_{a A}+n_{a B}\right)}{\Sigma_{5}\left(n_{A A}+n_{A B}+n_{B B}\right)}
$$

$$
\begin{aligned}
& d_{A B}=d_{A B}^{u p}+c n_{A B}\left(n_{a B}+n_{a A}\right), \\
& \dot{n}_{A B} \leq \dot{n}_{A B}^{u p}+\frac{f}{\Sigma_{5}} n_{a A}\left(\frac{1}{2} n_{a B}+\frac{1}{2} n_{A B}+n_{B B}\right)+\frac{f}{\Sigma_{5}} n_{a B} n_{A A}+\frac{f}{2 \Sigma_{5}} n_{a B} n_{A B}, \\
& \dot{n}_{A B} \geq \dot{n}_{A B}^{u p}-\frac{f\left(\frac{1}{2} n_{a B}+\frac{1}{2} n_{A B}+n_{B B}\right)}{\Sigma_{5} \Sigma_{6}} n_{a a} n_{A A}-\frac{2 f\left(\frac{1}{2} n_{A B}+n_{A A}\right)\left(\frac{1}{2} n_{A B}+n_{B B}\right)}{\Sigma_{5}\left(n_{A A}+n_{A B}+n_{B B}\right)}\left(n_{a A}+n_{a B}\right)-c n_{A B}\left(n_{a B}+n_{a A}\right) .
\end{aligned}
$$

And finally for the $A A$-population:

$$
\begin{aligned}
b_{A A}= & \frac{f\left(\frac{1}{2} n_{A B}+n_{A A}\right)^{2}}{\Sigma_{5}}+\frac{f n_{a A} n_{A B}}{4 \Sigma_{5}}-\frac{f n_{a a} n_{A A}\left(\frac{1}{2} n_{a A}+n_{A A}+\frac{1}{2} n_{A B}\right)}{\Sigma_{5} \Sigma_{6}}+\frac{f n_{a A}\left(\frac{1}{2} n_{a A}+n_{A A}+\frac{1}{2} n_{A B}\right)}{2 \Sigma_{6}} \\
= & b_{A A}^{u p}-\frac{f\left(\frac{1}{2} n_{A B}+n_{A A}\right)^{2}\left(n_{a A}+n_{a B}\right)}{\Sigma_{5}\left(n_{A A}+n_{A B}+n_{B B}\right)}+\frac{f n_{a A} n_{A B}}{4 \Sigma_{5}}-\frac{f n_{a a} n_{A A}\left(\frac{1}{2} n_{a A}+n_{A A}+\frac{1}{2} n_{A B}\right)}{\Sigma_{5} \Sigma_{6}} \\
& +\frac{f n_{a A}\left(\frac{1}{2} n_{a A}+n_{A A}+\frac{1}{2} n_{A B}\right)}{2 \Sigma_{6}}, \\
d_{A A}= & d_{A A}^{u p}+c n_{A A}\left(n_{a a}+n_{a A}+n_{a B}\right), \\
\dot{n}_{A A} \leq & \dot{n}_{A A}^{u p}+\frac{f n_{a A} n_{A B}}{4 \Sigma_{5}}+\frac{f n_{a A}\left(\frac{1}{2} n_{a A}+n_{A A}+\frac{1}{2} n_{A B}\right)}{2 \Sigma_{6}}, \\
\dot{n}_{A A} \geq & \dot{n}_{A A}^{u p}-\frac{f\left(\frac{1}{2} n_{a A}+\frac{1}{2} n_{A B}+n_{A A}\right)^{2}\left(n_{a a}+n_{a A}+n_{a B}\right)}{\Sigma_{5}\left(n_{A A}+n_{A B}+n_{B B}\right)}-c n_{A A}\left(n_{a a}+n_{a A}+n_{a B}\right) .
\end{aligned}
$$

As solutions of a dynamical system are continuous with respect to its parameters (in particular with respect to $\delta$ ), the latter theorem shows that until $T_{2}$, the 3 -system $(A A, A B, B B)$ is at most perturbed by $\Theta(\delta)$. We will show that $T_{2}$ diverges with $\varepsilon$. Thus, for small enough $\delta, A B$ will have time to reach the small fixed value $\sqrt{\varepsilon_{0}}>0$ in this phase, and we can use the asymptotic decay of the $A B$ and $A A$ populations which is proved in [25]. We now start to analyse the growth of the small $a a-, a A$ - and $a B$-populations. The sum-process $\Sigma_{5}$ plays a crucial role for the behaviour of the system in this phase and we need finer bounds on it:

Proposition 4.6. The sum-process $\Sigma_{5}=n_{a A}+n_{A A}+n_{a B}+n_{A B}+n_{B B}$ satisfies for all $t \in\left[T_{1}, T_{2}\right]:$

$$
\bar{n}_{B}-\frac{\Delta}{c \bar{n}_{B}} n_{A A}-\frac{\Delta^{2}}{c \bar{n}_{B}} n_{A A} \leq \Sigma_{5} \leq \bar{n}_{B}-\frac{\Delta}{c \bar{n}_{B}} n_{A A}+\frac{\Delta^{2}}{c \bar{n}_{B}} n_{A A} .
$$

Proof. We estimate a minorising process and a majorising process on $\Sigma_{5}$ :

$$
\begin{aligned}
b_{\Sigma_{5}} \leq & f \frac{\left(n_{A A}+n_{A B}+n_{B B}\right)\left(n_{a A}+n_{A A}+n_{a B}+n_{A B}+n_{B B}\right)}{n_{a A}+n_{A A}+n_{a B}+n_{A B}+n_{B B}} \\
& +f \frac{\left(n_{a A}+n_{a B}\right)\left(\frac{3}{4} n_{a A}+n_{A A}+\frac{3}{4} n_{a B}+n_{A B}+n_{B B}\right)}{n_{a A}+n_{A A}+n_{a B}+n_{A B}+n_{B B}}+\Theta(\delta) \leq f \Sigma_{5}+\Theta(\delta), \\
b_{\Sigma_{5}} \geq & f \frac{\left(n_{A A}+n_{A B}+n_{B B}\right)\left(n_{a A}+n_{A A}+n_{a B}+n_{A B}+n_{B B}\right)}{n_{a A}+n_{A A}+n_{a B}+n_{A B}+n_{B B}}
\end{aligned}
$$




$$
\begin{aligned}
& +f \frac{\left(n_{a A}+n_{a B}\right)\left(\frac{3}{4} n_{a A}+n_{A A}+\frac{3}{4} n_{a B}+n_{A B}+n_{B B}\right)}{n_{a A}+n_{A A}+n_{a B}+n_{A B}+n_{B B}}-\Theta(\delta) \geq f \Sigma_{5}-\Theta(\delta), \\
& d_{\Sigma_{5}} \leq \Sigma_{5}\left(D-\Delta+c \Sigma_{5}\right)+\Delta\left(n_{A A}+n_{a A}\right)-2 \eta n_{a A} n_{B B}+\Theta(\delta), \\
& d_{\Sigma_{5}} \geq \Sigma_{5}\left(D-\Delta+c \Sigma_{5}\right)+\Delta\left(n_{A A}+n_{a A}\right)-2 \eta n_{a A} n_{B B} .
\end{aligned}
$$

We get

$$
\begin{aligned}
& \dot{\Sigma}_{5} \leq-c \Sigma_{5}^{2}+\Sigma_{5}(f-D+\Delta)-\Delta n_{A A}+\Theta(\delta), \\
& \dot{\Sigma}_{5} \geq-c \Sigma_{5}^{2}+\Sigma_{5}(f-D+\Delta)-\Delta n_{A A}-\Theta(\delta) .
\end{aligned}
$$

We start with the proof of the upper bound. We use Lemma 4.2 and show that when $\Sigma_{5}$ reaches the upper-bound, it decays faster than the latter. Using (4.71) we compute $\dot{\Sigma}_{5}$ at the bound. Note that if $\Sigma_{5} \leq \bar{n}_{B}-\frac{\Delta}{c \bar{n}_{B}} n_{A A}+\frac{\Delta^{2}}{c \bar{n}_{B}} n_{A A}$, then $\Sigma_{5}^{2} \leq \bar{n}_{B}^{2}-\frac{2 \Delta}{c} n_{A A}+\frac{\Delta^{2}}{c^{2} \bar{n}_{B}^{2}} n_{A A}^{2}+\frac{2 \Delta^{2}}{c} n_{A A}+$ $\Theta\left(\Delta^{4}\right) n_{A A}^{2}$, thus

$$
\dot{\Sigma}_{5} \leq-\Delta^{2} n_{A A}-\frac{\Delta^{2}}{c \bar{n}_{B}^{2}} n_{A A}^{2}+\Theta(\delta)<0 .
$$

It is left to show that $\dot{\Sigma}_{5} \leq-\frac{\Delta}{c \bar{n}_{B}} \dot{n}_{A A}+\frac{\Delta^{2}}{c \bar{n}_{B}} \dot{n}_{A A}$. Since we already know (cf. Lemma 4.5 ) that $(A A, A B, B B)$ behaves like a 3 -system with $\Theta(\delta)$ perturbations, then $A A$ is decreasing, $\dot{n}_{A A} \leq 0$, this finishes the proof of the upper bound.

Now we check the lower bound. If $\Sigma_{5} \geq \bar{n}_{B}-\frac{\Delta}{c \bar{n}_{B}} n_{A A}-\frac{\Delta^{2}}{c \bar{n}_{B}} n_{A A}$ then $\Sigma_{5}^{2} \geq \bar{n}_{B}^{2}-\frac{2 \Delta}{c} n_{A A}-$ $\frac{\Delta^{2}}{c^{2} \bar{n}_{B}^{2}} n_{A A}-\frac{2 \Delta^{2}}{c} n_{A A}$. Using (4.72), the derivative of $\Sigma_{5}$ at the lower bound is thus lower bounded by

$$
\dot{\Sigma}_{5} \geq \Delta^{2} n_{A A}-\frac{\Delta^{2}}{c \bar{n}_{B}^{2}} n_{A A}-\Theta(\delta) \geq \Delta^{2} n_{A A}\left(1-\frac{1}{c \bar{n}_{B}}\right)-\Theta(\delta)>0 .
$$

By Lemma 4.2, it is enough to show that at the lower bound $\dot{\Sigma}_{5} \geq-\frac{\Delta}{c \bar{n}_{B}} \dot{n}_{A A}$. For this we calculate a majorising process on $A A$ :

$$
\begin{aligned}
& b_{A A} \leq \frac{f}{\Sigma_{5}} n_{A A}\left(n_{A A}+n_{A B}\right)+\frac{f}{4 \Sigma_{5}} n_{A B}^{2}+\Theta(\delta), \\
& d_{A A} \geq f n_{A A}, \\
& \dot{n}_{A A} \leq-\frac{f}{\Sigma_{5}} n_{A A} n_{B B}+\frac{f}{4 \Sigma_{5}} n_{A B}^{2}+\Theta(\delta) .
\end{aligned}
$$

Hence we have to show that $\Delta^{2} n_{A A}\left(1-\frac{1}{c \bar{n}_{B}}\right)-\Theta(\delta) \geq \frac{\Delta f}{c \bar{n}_{B} \bar{n}_{A}}\left(n_{A A} n_{B B}-\frac{1}{4} n_{A B}^{2}\right)-\Theta(\delta \Delta)$, in the case $n_{A A} n_{B B}>\frac{1}{4} n_{A B}^{2}$. This is equivalent to show that $\chi:=n_{A A} n_{B B}-\frac{1}{4} n_{A B}^{2} \leq$ $\frac{\Delta \bar{n}_{A}}{f}\left(c \bar{n}_{B}-1\right) n_{A A}$. For this we use once again Lemma 4.2 and estimate the derivative of $\chi$ from above with the help of minorising processes on $A A$ and $B B$ and a majorising process on $A B$ :

$$
\begin{aligned}
b_{A A} & \geq \frac{f}{\Sigma_{5}} n_{A A}\left(n_{A A}+n_{A B}\right)+\frac{f}{4 \Sigma_{5}} n_{A B}^{2}-\Theta(\delta), \\
d_{A A} & \leq(f+\Delta) n_{A A}+\Theta(\delta), \\
\dot{n}_{A A} & \geq-\frac{f}{\Sigma_{5}} n_{A A} n_{B B}-\Delta n_{A A}+\frac{f}{4 \Sigma_{5}} n_{A B}^{2}-\Theta(\delta) . \\
b_{B B} & \geq \frac{f}{\Sigma_{5}} n_{B B}\left(n_{A B}+n_{B B}\right)+\frac{f}{4 \Sigma_{5}} n_{A B}^{2}-\Theta(\delta), \\
d_{B B} & \leq f n_{B B}, \\
\dot{n}_{B B} & \geq-\frac{f}{\Sigma_{5}} n_{A A} n_{B B}+\frac{f}{4 \Sigma_{5}} n_{A B}^{2}+\Theta(\delta) . \\
b_{A B} & \leq \frac{f}{\Sigma_{5}} n_{A B}\left(n_{A A}+\frac{1}{2} n_{A B}+n_{B B}\right)+\frac{2 f}{\Sigma_{5}} n_{A A} n_{B B}+\Theta(\delta), \\
d_{A B} & \geq(f-\Delta) n_{A B},
\end{aligned}
$$




$$
\dot{n}_{A B} \leq \frac{2 f}{\Sigma_{5}} n_{A A} n_{B B}-\frac{f}{2 \Sigma_{5}} n_{A B}^{2}+\Delta n_{A B}+\Theta(\delta) .
$$

The derivative is given by:

$$
\begin{aligned}
\dot{\chi} & =\dot{n}_{A A} n_{B B}+n_{A A} \dot{n}_{B B}-\frac{1}{2} \dot{n}_{A B} n_{A B} \\
& \leq-f \chi+\Theta(\delta) .
\end{aligned}
$$

At the upper bound we get:

$$
\dot{\chi} \leq-\Delta \bar{n}_{A}\left(c \bar{n}_{B}-1\right) n_{A A}+\Theta(\delta)<0 .
$$

It is left to show that $\dot{\chi} \leq \frac{\Delta \bar{n}_{A}}{f}\left(c \bar{n}_{B}-1\right) \dot{n}_{A A}$. Using the minorising process $\dot{n}_{A A} \geq-\Delta n_{A A}-$ $\frac{f}{\bar{n}_{A}} \chi-\Theta(\delta)$ we show that

$$
0 \leq(f-2 \Delta) \chi-\frac{\Delta \bar{n}_{A}}{\bar{n}_{B}}\left(c \bar{n}_{B}-1\right) \chi-\frac{\Delta^{2} \bar{n}_{A}}{f}\left(c \bar{n}_{B}-1\right) n_{A A}-\Theta(\delta) .
$$

An easy calculation proves this fact and finishes the proof of the lower bound.

Lemma 4.7. For $t \in\left[T_{1}, T_{2}\right]$ and for $\Delta$ sufficiently small it holds,

$$
\dot{\Sigma}_{a A, a B} \geq-\Theta(\Delta) \Sigma_{a A, a B} .
$$

Proof. Using Propositions 4.6, we have the following bound on the process:

$$
\begin{aligned}
b_{\Sigma_{a A, a B}} & \geq f \frac{n_{a A}\left(\frac{1}{2} n_{a A}+n_{A A}+n_{a B}+n_{A B}+n_{B B}\right)+n_{a B}\left(n_{A A}+\frac{1}{2} n_{a B}+n_{A B}+n_{B B}\right)}{n_{a A}+n_{A A}+n_{a B}+n_{A B}+n_{B B}}-\Theta\left(\delta n_{a A}\right) \\
& \geq f \Sigma_{a A, a B}-\Theta\left(\delta n_{a A}\right), \\
d_{\Sigma_{a A, a B}} & =\Sigma_{a A, a B}\left(D-\Delta+c \Sigma_{5}\right)-\eta n_{a A} n_{B B}+\Delta n_{a A}+c n_{a A} n_{a a} \\
& \leq f \Sigma_{a A, a B}-n_{a A}\left(\eta n_{B B}-\Delta\right)+\Theta\left(\Delta^{2} n_{A A}\right) \Sigma_{a A, a B}^{2} \\
\dot{\Sigma}_{a A, a B} & \geq n_{a A}\left(\eta n_{B B}-\Delta-\Theta(\delta)\right) \Theta\left(\Delta^{2} n_{A A}\right) \Sigma_{a A, a B}^{2} \geq n_{a A}(-\Delta-\Theta(\delta))-\Theta\left(\delta \Delta^{2} n_{A A}\right) \Sigma_{a A, a B} \\
& \geq \Sigma_{a A, a B}(-\Delta-\Theta(\delta)) .
\end{aligned}
$$

Lemma 4.8. For all $t \in\left[T_{1}, T_{2}\right]$ the aa-population is bounded by

$$
\frac{f}{4 \bar{n}_{B}(f+\Delta)} \Sigma_{a A, a B}^{2} \leq n_{a a} \leq \frac{f}{\bar{n}_{A}(D+\Delta)} \Sigma_{a A, a B}^{2} .
$$

Observe that this implies $T_{2}=T^{a A=\delta A A} \wedge T^{a B=\delta A B}$.

Proof. First observe that the inequality is satisfied at $t=T_{1}$. We start with the upper bound and show that $n_{a a}$ would decrease at this bound. For this we estimate a majorising process on $a a$ :

$$
\begin{aligned}
& b_{a a} \leq \frac{f}{n_{a a}+n_{a A}+n_{A A}} n_{a a}\left(\frac{1}{2} n_{a A}+n_{a a}\right)+\frac{f}{4 \Sigma_{5}} \Sigma_{a A, a B}^{2}+\frac{f}{2 \Sigma_{5}} n_{a A} n_{a a}, \\
& d_{a a} \geq n_{a a}(D+\Delta), \\
& \dot{n}_{a a} \leq \frac{f}{n_{a a}+n_{a A}+n_{A A}} n_{a a}^{2}+\frac{f}{n_{a a}+n_{a A}+n_{A A}} n_{a a} n_{a A}+\frac{f}{4 \Sigma_{5}} \Sigma_{a A, a B}^{2}-n_{a a}(D+\Delta) .
\end{aligned}
$$

We calculate the slope of this process at the upper bound:

$$
\dot{n}_{a a} \leq \frac{f}{4 \Sigma_{5}} \Sigma_{a A, a B}^{2}-\frac{f}{\bar{n}_{A}} \Sigma_{a A, a B}^{2}+\Theta\left(\Sigma_{a A, a B}^{2} n_{a A}\right) \leq-\frac{3 f-\Theta(\delta)}{4 \bar{n}_{A}} \Sigma_{a A, a B}^{2}<0 .
$$

By Lemma 4.2, to ensure that 4.95) stays an upper bound it is enough to show that

$$
-\frac{3 f-\Theta(\delta)}{4 \bar{n}_{A}} \Sigma_{a A, a B}^{2} \leq \frac{2 f}{\bar{n}_{A}(D+\Delta)} \dot{\Sigma}_{a A, a B} \Sigma_{a A, a B} .
$$


This is a consequence of Lemma 4.7 .

For the lower bound we proceed similarly. This time, with the knowledge of the upper bound, we estimate a minorising process on $a a$ :

$$
\begin{aligned}
& b_{a a} \geq \frac{f}{\Sigma_{5}} \Sigma_{a A, a B}^{2}-\Theta\left(\Sigma_{a A, a B}^{3}\right), \\
& d_{a a} \leq n_{a a}(f+\Delta), \\
& \dot{n}_{a a} \geq \frac{f}{\bar{n}_{B}} \Sigma_{a A, a B}^{2}-n_{a a}(f+\Delta)-\Theta\left(\Sigma_{a A, a B}^{3}\right) .
\end{aligned}
$$

At the lower bound the process increases:

$$
\dot{n}_{a a} \geq\left(\frac{f}{\bar{n}_{B}}-\frac{f}{4 \bar{n}_{B}}\right) \Sigma_{a A, a B}^{2}-\Theta\left(\Sigma_{a A, a B}^{3}\right)=\frac{3 f}{4 \bar{n}_{B}} \Sigma_{a A, a B}^{2}-\Theta\left(\Sigma_{a A, a B}^{3}\right)>0 .
$$

By Lemma 4.2 it is left to show that $\dot{n}_{a a} \geq \frac{f}{2 \bar{n}_{B}(f+\Delta)} \dot{\Sigma}_{a A, a B} \Sigma_{a A, a B}$. Thus we have to calculate a majorising process on $\Sigma_{a A, a b}$ :

$$
\begin{aligned}
b_{\Sigma_{a A, a B}} & \leq f \Sigma_{a A, a B}+\Theta\left(\Sigma_{a A, a B}^{2}\right) \\
d_{\Sigma_{a A, a B}} & \geq(f-\Delta) \Sigma_{a A, a B}+n_{a A}\left(\Delta-\eta n_{B B}\right) \\
& \geq(f-\Delta) \Sigma_{a A, a B}-(f-D) \Sigma_{a A, a B} \\
& =(D-\Delta) \Sigma_{a A, a B}, \\
\dot{\Sigma}_{a A, a B} & \leq(f-D+\Delta) \Sigma_{a A, a B}+\Theta\left(\Sigma_{a A, a B}^{2}\right) .
\end{aligned}
$$

Thus we get

$$
\begin{aligned}
\frac{f(f-D+\Delta)}{2 \bar{n}_{B}(f+\Delta)} \Sigma_{a A, a B}^{2}-\frac{3 f}{4 \bar{n}_{B}} \Sigma_{a A, a B}^{2}+\Theta\left(\Sigma_{a A, a B}^{3}\right) & =-\frac{f}{2 \bar{n}_{B}} \Sigma_{a A, a B}^{2}\left(\frac{3}{2}-\frac{f-D+\Delta}{f+\Delta}\right)+\Theta\left(\Sigma_{a A, a B}^{3}\right) \\
& =-\frac{f}{2 \bar{n}_{B}} \Sigma_{a A, a B}^{2} \frac{f+2 D+\Delta}{2(f+\Delta)}+\Theta\left(\Sigma_{a A, a B}^{3}\right)<0
\end{aligned}
$$

This finishes the proof of the lower bound.

Let

$$
T_{\equiv}=\inf \left\{t>T_{1}: n_{a A}(t)=n_{a B}(t)\right\} .
$$

Proposition 4.9. For all $t \in\left[T_{1}, T_{=}\right]$it holds

$$
n_{a B} \leq n_{a A}=\Theta(\varepsilon) \text {. }
$$

Proof. In this time interval the newborns of genotype $a A$ are in majority produced by reproductions of a population of order one, namely $A B$ or $A A$, with the population $a A$. Since $n_{a A}$ feels competition from a macroscopic population $(A A, A B$ or $B B)$ the $a A$-population stays of order $\Theta(\varepsilon)$. We make this more rigorous. To show this we consider a majorising process on $a A$ and use Proposition 4.6, and Lemma 4.8.

$$
\begin{aligned}
b_{a A} & \leq f n_{a A}-\frac{f}{\Sigma_{5}} n_{a A}\left(n_{B B}+\frac{1}{2} n_{A B}\right)+\frac{f}{2 \Sigma_{5}} n_{a B}\left(2 n_{A A}+n_{A B}\right)+\Theta\left(\Sigma_{a A, a B}^{2}\right), \\
d_{a A} & \geq n_{a A}\left(f+\Delta-\frac{\Delta}{\bar{n}_{B}} n_{A A}-\eta n_{B B}-\Theta\left(\Delta^{2} n_{A A}\right)\right), \\
\dot{n}_{a A} & \leq-n_{a A}\left(n_{B B} \frac{f-\eta \Sigma_{5}}{\Sigma_{5}}+\frac{f}{2 \Sigma_{5}} n_{A B}+\Delta\left(1-\frac{n_{A A}}{\bar{n}_{B}}\right)-\Theta\left(\Delta^{2} n_{A A}\right)\right)+\frac{f}{\Sigma_{5}} n_{a B}\left(\frac{1}{2} n_{A B}+n_{A A}+\Theta(\delta)\right) \\
& \leq-n_{a A}\left(n_{B B} \frac{D+\Delta}{\Sigma_{5}}+\frac{f}{2 \Sigma_{5}} n_{A B}+\Delta\left(1-\frac{n_{A A}}{\bar{n}_{B}}\right)-\Theta\left(\Delta^{2} n_{A A}\right)\right)+\frac{f}{\Sigma_{5}} n_{a B}\left(\frac{1}{2} n_{A B}+n_{A A}+\Theta(\delta)\right) \\
& \leq-n_{a A}\left(\frac{f}{\Sigma_{5}}\left(\frac{D+\Delta}{f} n_{B B}+\frac{1}{2} n_{A B}\right)+\Delta\left(1-\frac{n_{A A}}{\bar{n}_{B}}\right)-\Theta\left(\Delta^{2} n_{A A}\right)\right)+\frac{f}{\Sigma_{5}} n_{a B}\left(\frac{1}{2} n_{A B}+n_{A A}+\Theta(\delta)\right) .
\end{aligned}
$$


By Proposition 4.5 and [25] there exists a time $t_{0}=\Theta(1)$ such that the expression in the first bracket becomes bigger than the expression in the second bracket. Thus $n_{a A}$ decreases after $t_{0}$ and since $a A$ does not exceed $\Theta(\varepsilon)$ until $t_{0}$ it will stay smaller or equal to $\Theta(\varepsilon)$ until $T_{=}$.

We show that as soon as $a B$ crosses $a A$ the $B B$-population is already bigger than or equal to the $A A$-population. First we estimate a upper bound for $a B$ :

Lemma 4.10. For all $t \in\left[T_{1}, T_{2}\right]$ the aB-population is upper bounded by

$$
n_{a B} \leq \frac{n_{A B}+2 n_{B B}+\frac{2 \Delta}{c}}{n_{A B}+2 n_{A A}} n_{a A} \equiv C(t) n_{a A} .
$$

Proof. First observe that the bound is fulfilled at $t=T_{1}$. Similarly to the proof of Lemma 4.8 we estimate a majorising process on $a B$ given by:

$$
\dot{n}_{a B} \leq-n_{a B}\left(\frac{f}{2 \Sigma_{5}}\left(n_{A B}+2 n_{A A}\right)-\frac{\Delta}{\bar{n}_{B}} n_{A A}-\Theta\left(\Delta^{2} n_{A A}\right)\right)+n_{a A} \frac{f}{2 \Sigma_{5}}\left(n_{A B}+2 n_{B B}+\Theta(\delta)\right) .
$$

By Lemma 4.2, we have to show that as soon as $a B$ reaches the upper bound it decreases faster than the bound, thus we calculate the slope of the majorising process at this value:

$$
\begin{aligned}
\dot{n}_{a B} & \leq-\frac{f}{2 \Sigma_{5}}\left(n_{A B}+2 n_{B B}+\frac{2 \Delta}{c}-\Theta\left(\Delta^{2} n_{A A}\right)\right) n_{a A}+\frac{\Delta\left(n_{A B}+2 n_{B B}+2 \Delta / c\right)}{\bar{n}_{B}\left(n_{A B}+2 n_{A A}\right)} n_{A A} n_{a A}+\frac{f}{2 \Sigma_{5}}\left(n_{A B}+2 n_{B B}\right) n_{a A} \\
& \leq-\frac{\Delta f-\Theta\left(\Delta^{2} n_{A A}\right)}{c \Sigma_{5}} n_{a A}+\frac{\Delta}{\Sigma_{5}}\left(\frac{1}{2} n_{A B}+n_{B B}+\frac{\Delta}{c}\right) n_{a A} \\
& \leq \frac{\Delta+\Theta\left(\Delta^{2} n_{A A}\right)}{\Sigma_{5}} n_{a A}\left(\bar{n}_{B}+\frac{\Delta}{c}-\frac{f}{c}\right) \\
& \leq-\frac{\Delta+\Theta\left(\Delta^{2} n_{A A}\right)}{c \Sigma_{5}}(D-2 \Delta) n_{a A} \leq 0 .
\end{aligned}
$$

We have to show that $\dot{n}_{a B} \leq C(t) \dot{n}_{a A}+\dot{C}(t) n_{a A}$. Since the 3 -system converges towards $\left(0,0, \bar{n}_{B}\right), C(t)$ is a monotone increasing function and hence $\dot{C}(t) \geq 0$. Thus if we can show that $\dot{n}_{a B} \leq C(t) \dot{n}_{a A}$ we are done. For this we have to calculate the slope of the minorising process on $a A$ when $a B$ would reach the upper bound. This process is given by:

$$
\dot{n}_{a A} \geq-n_{a A}\left(\frac{f}{2}+\Delta-\eta n_{B B}+\frac{f}{2 \Sigma_{5}}\left(n_{B B}-n_{A A}\right)+\Theta(\delta)\right)+n_{a B} \frac{f}{2 \Sigma_{5}}\left(n_{A B}+2 n_{A A}\right) .
$$

The slope at the upper bound is:

$$
\begin{aligned}
\dot{n}_{a A} & \geq-n_{a A}\left(\frac{f}{2}+\Delta-\eta n_{B B}+\frac{f}{2 \Sigma_{5}}\left(n_{B B}-n_{A A}\right)-\frac{f}{2 \Sigma_{5}}\left(n_{A B}+2 n_{B B}+\frac{2 \Delta}{c}+\Theta(\delta)\right)\right) \\
& \geq-n_{a A}\left(\Delta-\eta n_{B B}-\frac{\Delta f}{c \Sigma_{5}}+\Theta(\delta)\right) \\
& \geq n_{a A}\left(\Delta \frac{D-\Delta}{c \Sigma_{5}}+\eta n_{B B}-\Theta(\delta)\right) \geq 0 .
\end{aligned}
$$

Since $C(t)>0$ this finishes the proof.

Lemma 4.11. We have $T_{=} \leq T_{2}$. Moreover it holds,

$$
n_{A A}\left(T_{=}\right) \leq n_{B B}\left(T_{=}\right)+\Theta(\Delta) .
$$

Proof. We first show that $T_{=}<T_{2}$. Using Proposition 4.6 we construct two processes that provide an upper bound and a lower bound on $n_{a B}$, respectively:

$$
\begin{aligned}
b_{a B} & \geq f n_{a B}-\frac{f}{\Sigma_{5}} n_{a B}\left(\frac{1}{2} n_{A B}+n_{A A}\right)+\frac{f}{\Sigma_{5}} n_{a A}\left(\frac{1}{2} n_{A B}+n_{B B}-\Theta\left(\delta^{2}\right)\right), \\
b_{a B} & \leq f n_{a B}-\frac{f}{\Sigma_{5}} n_{a B}\left(\frac{1}{2} n_{A B}+n_{A A}\right)+\frac{f}{\Sigma_{5}} n_{a A}\left(\frac{1}{2} n_{A B}+n_{B B}+\Theta(\delta)\right), \\
d_{a B} & \leq n_{a B} f, \\
d_{a B} & \geq n_{a B}\left(f-\frac{\Delta}{\bar{n}_{B}} n_{A A}-\Theta\left(\Delta^{2} n_{A A}\right)\right),
\end{aligned}
$$




$$
\begin{aligned}
& \dot{n}_{a B} \leq-n_{a B}\left(\frac{f\left(\frac{1}{2} n_{A B}+n_{A A}\right)}{\Sigma_{5}}-\frac{\Delta}{\bar{n}_{B}} n_{A A}-\Theta\left(\Delta^{2} n_{A A}\right)\right)+n_{a A} \frac{f\left(\frac{1}{2} n_{A B}+n_{B B}+\Theta(\delta)\right)}{\Sigma_{5}}, \\
& \dot{n}_{a B} \geq-n_{a B} \frac{f\left(\frac{1}{2} n_{A B}+n_{A A}\right)}{\Sigma_{5}}+n_{a A} \frac{f\left(\frac{1}{2} n_{A B}+n_{B B}-\Theta\left(\delta^{2}\right)\right)}{\Sigma_{5}} .
\end{aligned}
$$

We first show that $T_{=}<\infty$. We know that the 3 -system $(A A, A B, B B)$ converges to $\left(0,0, \bar{n}_{B}\right)$ and that $n_{a B} \leq n_{a A}=\Theta(\varepsilon)$ (Proposition 4.9), for $t \leq T_{=}$. We consider the worst case and assume that $n_{a B}<n_{a A}$ then we get from 4.135) that at some time $t_{0}$, where $n_{A B}+2 n_{B B}$ is already macroscopic,

$$
\dot{n}_{a B} \geq \Theta(\varepsilon), \quad n_{a B} \geq \Theta(\varepsilon) t .
$$

Thus the time $a B$ needs to reach $n_{a A}=\Theta(\varepsilon)$ is of order $\Theta(1)$. This time is shorter than $T_{a A=\delta A A}$. Indeed, suppose the contrary, then by Proposition $4.9 n_{a A}$ does not exceed $\Theta(\varepsilon)$ before $T_{2}$, and thus $T^{a A=\delta A A} \geq T_{\Theta(\varepsilon / \delta)}^{A A}=\Theta\left((\delta / \varepsilon)^{2}\right)$ which diverges with $\varepsilon$. A similar reasoning shows that $T_{=}<T^{a B=\delta A B}$. Hence $T_{=}<T_{2}$.

It is left to show that $n_{A A}\left(T_{=}\right) \leq n_{B B}\left(T_{=}\right)+\Theta(\Delta)$. From Lemma 4.10 we deduce that at $T_{=}$ it holds

$$
\begin{aligned}
\frac{1}{2} n_{A B}+n_{A A} & \leq \frac{1}{2} n_{A B}+n_{B B}+\frac{\Delta}{c} \\
n_{A A} & \leq n_{B B}+\Theta(\Delta) .
\end{aligned}
$$

Lemma 4.12. For all $t \in\left[T_{1}, T_{2}\right]$ the AB-population is bounded by

(1) $n_{A B} \geq 2 \sqrt{\bar{n}_{B} n_{A A}}-2 n_{A A}\left(1+\frac{\Delta}{c \bar{n}_{B}}\right)$,

(2) $n_{A B} \leq 2 \sqrt{\bar{n}_{B} n_{A A}\left(1+\frac{\Delta}{f}\right)}-2 n_{A A}$.

Proof.

(1) The proof works like the one of Lemma 4.8. First observe that the bound holds at $t=T_{1}$. Then we calculate a minorising process on $A B$ :

$$
\begin{aligned}
& b_{A B} \geq f\left(2 n_{A A}+n_{A B}\right)-\frac{f}{\Sigma_{5}}\left(2 n_{A A}+n_{A B}\right)\left(n_{A A}+\frac{1}{2} n_{A B}+\Theta\left(\delta^{2}\right)\right), \\
& d_{A B} \leq f n_{A B}, \\
& \dot{n}_{A B} \geq-n_{A B} \frac{f}{\Sigma_{5}}\left(\frac{1}{2} n_{A B}+n_{A A}+\Theta\left(\delta^{2}\right)\right)+2 f n_{A A}-\frac{2 f}{\Sigma_{5}} n_{A A}\left(\frac{1}{2} n_{A B}+n_{A A}+\Theta\left(\delta^{2}\right)\right) .
\end{aligned}
$$

We use Proposition 4.6 and show that this minorising process would increase quicker than the lower-bound if $A B$ reaches it:

$$
\begin{aligned}
\dot{n}_{A B} \geq & -\frac{2 f}{\Sigma_{5}}\left(\sqrt{\bar{n}_{B} n_{A A}}-n_{A A}\left(1+\frac{\Delta}{c \bar{n}_{B}}\right)\right)\left(\sqrt{\bar{n}_{B} n_{A A}}-\frac{\Delta}{c \bar{n}_{B}} n_{A A}+\Theta\left(\delta^{2}\right)\right) \\
& +2 f n_{A A}-\frac{2 f}{\Sigma_{5}} n_{A A}\left(\sqrt{\bar{n}_{B} n_{A A}}-\frac{\Delta}{c \bar{n}_{B}} n_{A A}\right) \\
\geq & \frac{2 f}{\Sigma_{5}} \frac{\Delta}{c \bar{n}_{B}} n_{A A}\left(2 \sqrt{\bar{n}_{B} n_{A A}}-n_{A A}\right)-\Theta\left(\Delta^{2}\right)>0 .
\end{aligned}
$$

It is left to show that at the lower bound,

$$
\dot{n}_{A B} \geq \frac{\bar{n}_{B} \dot{n}_{A A}}{\sqrt{\bar{n}_{B} n_{A A}}}-2 \dot{n}_{A A}\left(1+\frac{\Delta}{c \bar{n}_{B}}\right) .
$$

For this we calculate a majorising process on $A A$ :

$$
\begin{aligned}
b_{A A} & \leq \frac{f}{\Sigma_{5}} n_{A A}\left(n_{A A}+n_{A B}\right)+\frac{f}{4 \Sigma_{5}} n_{A B}^{2}+\Theta(\delta), \\
d_{A A} & \geq f n_{A A},
\end{aligned}
$$




$$
\dot{n}_{A A} \leq-n_{A A}\left(f-\frac{f}{\Sigma_{5}}\left(n_{A A}+n_{A B}\right)\right)+\frac{f}{4 \Sigma_{5}} n_{A B}^{2}+\Theta(\delta) .
$$

If we now insert the lower bound and use Proposition 4.6 we get

$$
\dot{n}_{A A} \leq-\frac{f}{\Sigma_{5}} \frac{\Delta}{c \bar{n}_{B}} n_{A A}\left(\sqrt{\bar{n}_{B} n_{A A}}-n_{A A}\right)+\Theta\left(\Delta^{2}\right)<0 .
$$

Thus (4.144) is fulfilled.

(2) First, observe that the upper bound is fullfiled at $t=T_{1}$. We then have to estimate a majorising process on $A B$ :

$$
\begin{aligned}
b_{A B} & \leq f\left(2 n_{A A}+n_{A B}\right)-f\left(2 n_{A A}+n_{A B}\right) \frac{n_{A A}+\frac{1}{2} n_{A B}}{\Sigma_{5}}+\Theta(\delta), \\
d_{A B} & \geq n_{A B}\left(D-\Delta+c \bar{n}_{B}-\frac{\Delta}{\bar{n}_{B}} n_{A A}-\Theta\left(\Delta^{2} n_{A A}\right)\right) \\
& \geq n_{A B}\left(f-\frac{\Delta}{\bar{n}_{B}} n_{A A}-\Theta\left(\Delta^{2} n_{A A}\right)\right), \\
\dot{n}_{A B} & \leq-\frac{f}{2 \bar{n}_{B}} n_{A B}^{2}-n_{A B} \frac{2 f-\Delta}{\bar{n}_{B}} n_{A A}+2 f n_{A A}-\frac{2 f}{\bar{n}_{B}} n_{A A}^{2}+\Theta\left(\Delta^{2} n_{A A}\right) .
\end{aligned}
$$

As before we calculate the slope of this majorising process if it would reach the upper bound:

$$
\dot{n}_{A B} \leq-\frac{2 \Delta}{\bar{n}_{B}} n_{A A}^{2}+\Theta\left(\Delta^{2} n_{A A}\right)<0 .
$$

By Lemma 4.2 we have to show that

$$
\dot{n}_{A B} \leq \dot{n}_{A A}\left(\frac{\bar{n}_{B}(1+\Delta / f)}{\sqrt{\bar{n}_{B} n_{A A}(1+\Delta / f)}}-2\right) .
$$

For this we calculate the slope of a minorising process on $A A$ given by

$$
\dot{n}_{A A} \geq-n_{A A}\left(f-\frac{f}{\Sigma_{5}}\left(n_{A A}+n_{A B}\right)+\Delta+\Theta\left(\delta^{2}\right)\right)+\frac{f}{4 \Sigma_{5}} n_{A B}^{2} .
$$

At the upper bound $A A$ would start to increases:

$$
\dot{n}_{A A} \geq \frac{\Delta}{\bar{n}_{B}} n_{A A}^{2}-\Theta\left(\delta^{2}\right)>0 .
$$

Thus we get

$$
\dot{n}_{A A}\left(\frac{\bar{n}_{B}(1+\Delta / f)}{\sqrt{\bar{n}_{B} n_{A A}(1+\Delta / f)}}-2\right)-\dot{n}_{A B} \geq \frac{\Delta(1+\Delta / f)}{\sqrt{\bar{n}_{B} n_{A A}(1+\Delta / f)}} n_{A A}^{2}-\Theta\left(\Delta^{2} n_{A A}\right)>0 .
$$

This finishes the proof of (2).

The following Proposition is a statement for the 3-system $(A A, A B, B B)$ but it holds also true until $T_{2}$ in the 6-system ( $\left.a a, a A, A A, a B, A B, B B\right)$ for $\delta<\Delta$.

Proposition 4.13. The maximal value $n_{A B}^{\max }$ of $n_{A B}$ in $\left[T_{1}, T_{2}\right]$ is bounded by

$$
\frac{\bar{n}_{B}}{2}-\Theta(\Delta) \leq n_{A B}^{\max } \leq \frac{\bar{n}_{B}}{2}+\Theta(\Delta) .
$$

Moreover, let $T_{A B}^{\max }$ be the time when $n_{A B}$ takes on its maximum, then $n_{A A}$ and $n_{B B}$ are bounded by

$$
\begin{aligned}
& \frac{\bar{n}_{B}}{4}-\Theta(\Delta) \leq n_{A A}\left(T_{A B}^{\max }\right) \leq \frac{\bar{n}_{B}}{4}+\Theta(\Delta), \\
& \frac{\bar{n}_{B}}{4}-\Theta(\Delta) \leq n_{B B}\left(T_{A B}^{\max }\right) \leq \frac{\bar{n}_{B}}{4}+\Theta(\Delta) .
\end{aligned}
$$


Proof. From Lemma 4.12 (1) we get that

$$
n_{A B} \geq 2 \sqrt{\bar{n}_{B} n_{A A}}-2 n_{A A}\left(1+\frac{\Delta}{c \bar{n}_{B}}\right),
$$

We look for the value of $A A$ where the expression on the right hand side takes on its minimum, thus we have to derivate $n_{A A}$ and set it to zero:

$$
\begin{aligned}
\frac{\bar{n}_{B}}{\sqrt{\bar{n}_{B} n_{A A}}-\left(2+\frac{\Delta}{c \bar{n}_{B}}\right)} & =0 \\
\bar{n}_{B}^{2} & =\left(4-4 \frac{\Delta}{c \bar{n}_{B}}+\Theta\left(\Delta^{2}\right)\right) \bar{n}_{B} n_{A A} \\
\frac{\bar{n}_{B}}{4}-\Theta(\Delta) & =n_{A A} .
\end{aligned}
$$

If we insert this in $n_{A B}$ we get the lower bound:

$$
n_{A B} \geq \frac{\bar{n}_{B}}{2}+\Theta(\Delta)
$$

For the upper bound on $n_{A B}$ we proceed similarly. Form Lemma4.12 (2) we get

$$
n_{A B} \leq 2 \sqrt{\bar{n}_{B} n_{A A}\left(1+\frac{\Delta}{f}\right)}-2 n_{A A} .
$$

Setting the derivation of the rhs to zero gives:

$$
\begin{aligned}
0 & =\frac{\bar{n}_{B}\left(1+\frac{\Delta}{f}\right)}{\sqrt{\bar{n}_{B} n_{A A}\left(1+\frac{\Delta}{f}\right)}}-2 \\
n_{A A} & =\frac{\bar{n}_{B}}{4}+\Theta(\Delta) .
\end{aligned}
$$

Finally we get

$$
n_{A B} \leq \frac{\bar{n}_{B}}{2}-\Theta(\Delta) \quad \text { and } \quad n_{A A}=\frac{\bar{n}_{B}}{4}-\Theta(\Delta) .
$$

Remark. Note that $n_{A A}=n_{B B} \pm \Theta(\Delta)=\frac{\bar{n}_{B}}{4} \pm \Theta(\Delta)$ as soon as $n_{A B}$ reaches its maximal value.

Proposition 4.14. For all $t \in\left[T_{1}, T_{2}\right]$,

$$
n_{a A} \leq \Theta(\varepsilon) \vee n_{a B} .
$$

Proof. For $t \leq T_{=}$this follows from Proposition 4.9. For $t>T_{=}$we show this by constructing a majorising process on $n_{a A}(t)$ :

$$
\begin{aligned}
b_{a A} & \leq f \frac{\left(n_{a A}+n_{a B}\right)\left(2 n_{A A}+n_{A B}+\Theta(\delta)\right)}{2 \Sigma_{5}} \\
& \leq \frac{f+\Theta(\delta)}{2}\left(n_{a A}+n_{a B}\right)+\frac{f\left(n_{A A}-n_{B B}\right)}{2 \bar{n}_{A}}\left(n_{a A}+n_{a B}\right), \\
d_{a A} & \geq n_{a A}\left(D+c \bar{n}_{B}-\frac{\Delta}{\bar{n}_{B}} n_{A A}-\eta n_{B B}-\Theta\left(\Delta^{2} n_{A A}\right)\right) \\
& \geq n_{a A}\left(f-\eta n_{B B}\right), \\
\dot{n}_{a A} & \leq-n_{a A}\left(\frac{f}{2}-\frac{f\left(n_{A A}-n_{B B}\right)}{2 \bar{n}_{A}}-\eta n_{B B}-\Theta(\delta)\right)+n_{a B}\left(\frac{f}{2}+\frac{f\left(n_{A A}-n_{B B}+\Theta(\delta)\right)}{2 \bar{n}_{A}}\right) .
\end{aligned}
$$

By Lemma 4.2, it is left to show that $\dot{n}_{a A} \leq \dot{n}_{a B}$ whenever $n_{a A}=n_{a B}$. At this upper bound we have $\dot{n}_{a A} \leq n_{a B}\left(\frac{f}{\bar{n}_{A}}\left(n_{A A}-n_{B B}\right)+\eta n_{B B}+\Theta(\delta)\right)$. We now calculate a minorising process on $n_{a B}$ :

$$
b_{a B} \geq \frac{f}{2 \Sigma_{5}}\left(n_{a A}+n_{a B}\right)\left(n_{a B}+n_{A B}+2 n_{B B}\right),
$$




$$
\begin{aligned}
& d_{a B} \leq n_{a B}\left(D-\Delta+c \bar{n}_{B}\right)=f n_{a B}, \\
& \dot{n}_{a B} \geq \frac{f}{2 \Sigma_{5}} n_{a A}\left(n_{a B}+n_{A B}+2 n_{B B}\right)-\frac{f}{2 \Sigma_{5}} n_{a B}\left(2 n_{A A}+2 n_{a A}-n_{a B}-n_{A B}\right) .
\end{aligned}
$$

Thus $\dot{n}_{a B} \geq \frac{f}{\Sigma_{5}} n_{a B}\left(n_{B B}-n_{A A}+n_{A B}\right)$ whenever $n_{a A}=n_{a B}$, and hence $\dot{n}_{a B}-\dot{n}_{a A} \geq \frac{f}{\bar{n}_{A}} n_{a B}\left(2 n_{B B}-\right.$ $\left.2 n_{A A}+\eta n_{B B}-\Theta(\Delta)\right)>0$ by Proposition 4.13 . This finishes the proof.

Now we show that the time $T^{a A=\delta A A}$ is finite and prove that it is smaller than or equal to $T^{a B=\delta B B}$. To estimate the order of magnitude of the time $T_{2}$ we need bounds on $n_{a A}$ which depends on $\Sigma_{a A, a B}$.

Lemma 4.15. For all $t \in\left[T_{1}, T_{2}\right]$ the aA-population is bounded by

$$
\frac{f\left(n_{A B}+2 n_{A A}\right)}{4 \bar{n}_{B}(f+\Delta)} \Sigma_{a A, a B} \leq n_{a A} \leq \frac{f\left(n_{A B}+2 n_{A A}\right)}{\bar{n}_{A}(D-2 \Delta)} \Sigma_{a A, a B} .
$$

Proof.

(1) We start with the upper bound. First observe that it holds at $t=T_{1}$. By Lemma 4.2 it is enough to show that if $n_{a A}$ would reach the upper bound it would decrease faster than the bound. Using Proposition 4.6 and that $\eta<c$ a majorising process on $a A$ is given by

$$
\begin{aligned}
& b_{a A} \leq \frac{f}{2 \Sigma_{5}} \Sigma_{a A, a B}\left(n_{A B}+2 n_{A A}+\Theta(\delta)\right), \\
& d_{a A} \geq n_{a A}\left(D+c \bar{n}_{B}-\frac{\Delta}{\bar{n}_{B}} n_{A A}-\eta n_{B B}-\Theta\left(\Delta^{2} n_{A A}\right)\right) \geq n_{a A}(D-2 \Delta), \\
& \dot{n}_{a A} \leq \frac{f\left(2 n_{A A}+n_{A B}+\Theta(\delta)\right)}{2 \Sigma_{5}} \Sigma_{a A, a B}-n_{a A}(D-2 \Delta) .
\end{aligned}
$$

We calculate the slope of the majorising process at the upper bound:

$$
\dot{n}_{a A} \leq f\left(2 n_{A A}+n_{A B}\right) \Sigma_{a A, a B}\left(\frac{1}{2 \Sigma_{5}}-\frac{1}{\bar{n}_{A}}+\Theta(\delta)\right) \leq-\frac{f}{2 \bar{n}_{A}}\left(2 n_{A A}+n_{A B}+\Theta(\delta)\right) \Sigma_{a A, a B} .
$$

We have to show that at the upper bound,

$$
\dot{n}_{a A} \leq \frac{f\left(\dot{n}_{A B}+2 \dot{n}_{A A}\right)}{\bar{n}_{A}(D-2 \Delta)} \Sigma_{a A, a B}+\frac{f\left(n_{A B}+2 n_{A A}\right)}{\bar{n}_{A}(D-2 \Delta)} \dot{\Sigma}_{a A, a B} .
$$

To do this we calculate minorising processes on $n_{A B}$ and $n_{A A}$ :

$$
\begin{aligned}
& b_{A B} \geq \frac{f}{\Sigma_{5}} n_{A B}\left(\frac{1}{2} n_{A B}+n_{A A}+n_{B B}\right)+\frac{2 f}{\Sigma_{5}} n_{A A}\left(n_{B B}-\Theta\left(\delta^{2}\right)\right), \\
& d_{A B} \leq n_{A B} f, \\
& \dot{n}_{A B} \geq-\frac{f}{2 \Sigma_{5}} n_{A B}^{2}+\frac{2 f}{\Sigma_{5}} n_{A A}\left(n_{B B}-\Theta\left(\delta^{2}\right)\right), \\
& b_{A A} \geq \frac{f}{\Sigma_{5}} n_{A A}\left(n_{A B}+n_{A A}-\Theta\left(\delta^{2}\right)\right)+\frac{f}{4 \Sigma_{5}} n_{A B}^{2}, \\
& d_{A A} \leq n_{A A}\left(f+\Delta+\Theta\left(\delta^{2}\right)\right), \\
& \dot{n}_{A A} \geq-n_{A A}\left(\frac{f}{\Sigma_{5}} n_{B B}+\Delta+\Theta\left(\delta^{2}\right)\right)+\frac{f}{4 \Sigma_{5}} n_{A B}^{2} .
\end{aligned}
$$

Hence we get that

$$
\dot{n}_{A B}+2 \dot{n}_{A A} \geq n_{A A}\left(\frac{2 f}{\Sigma_{5}} n_{B B}-\frac{2 f}{\Sigma_{5}} n_{B B}-2 \Delta-\Theta\left(\delta^{2}\right)\right)=-2\left(\Delta+\Theta\left(\delta^{2}\right)\right) n_{A A} .
$$

By Lemma 4.7, we know that $\dot{\Sigma}_{a A, a B} \geq-\Delta \Sigma_{a A, a B}$. Thus the right-hand side minus the left-hand side of (4.184) is lower-bounded by

$$
-\frac{2 f\left(\Delta+\Theta\left(\delta^{2}\right)\right) n_{A A} \Sigma_{a A, a B}}{\bar{n}_{A}(D-2 \Delta)}-\frac{f \Delta\left(n_{A B}+2 n_{A A}\right) \Sigma_{a A, a B}}{\bar{n}_{A}(D-2 \Delta)}+\frac{f\left(n_{A B}+2 n_{A A}+\Theta(\delta)\right) \Sigma_{a A, a B}}{2 \bar{n}_{A}}
$$




$$
\geq \frac{f n_{A A} \Sigma_{a A, a B}}{\bar{n}_{A}}\left(1-\frac{4 \Delta}{D-2 \Delta}\right)+\frac{f n_{A B} \Sigma_{a A, a B}}{2 \bar{n}_{A}}\left(1-\frac{2 \Delta}{D-2 \Delta}\right)+\Theta\left(\delta^{2}\right)>0 .
$$

This finishes the proof of (1).

(2) For the lower bound we proceed similarly (using Lemma 4.2). This time we show that if $n_{a A}$ would reach the lower bound it would start to increase faster than the bound. Using Proposition 4.6 a minorising process on $n_{a A}$ is given by

$$
\begin{aligned}
b_{a A} & \geq \frac{f}{2 \Sigma_{5}} \Sigma_{a A, a B}\left(2 n_{A A}+n_{A B}-\Theta(\delta)\right), \\
d_{a A} & \leq n_{a A}\left(f+\Delta+\Theta\left(\delta^{2}\right)\right), \\
\dot{n}_{a A} & \geq \frac{f\left(2 n_{A A}+n_{A B}-\Theta(\delta)\right)}{2 \bar{n}_{B}} \Sigma_{a A, a B}-n_{a A}(f+\Delta) .
\end{aligned}
$$

We calculate the slope of the minorising process at the lower bound:

$$
\begin{aligned}
\dot{n}_{a A} & \geq \frac{f\left(2 n_{A A}+n_{A B}-\Theta(\delta)\right)}{2 \bar{n}_{B}} \Sigma_{a A, a B}-\frac{f\left(2 n_{A A}+n_{A B}\right)}{4 \bar{n}_{B}} \Sigma_{a A, a B} \\
& =\frac{f\left(2 n_{A A}+n_{A B}-\Theta(\delta)\right)}{4 \bar{n}_{B}} \Sigma_{a A, a B}>0 .
\end{aligned}
$$

Thus the minorising process on $n_{a A}$ would increase when the $a A$-population would reach the lower bound. To ensure this lower bound we have to show

$$
\dot{n}_{a A} \geq \frac{f\left(\dot{n}_{A B}+2 \dot{n}_{A A}\right)}{4 \bar{n}_{B}(f+\Delta)} \Sigma_{a A, a B}+\frac{f\left(n_{A B}+2 n_{A A}\right)}{4 \bar{n}_{B}(f+\Delta)} \dot{\Sigma}_{a A, a B}
$$

For this we consider a majorising process on $\Sigma_{a A, a B}$ given by:

$$
\dot{\Sigma}_{a A, a B} \leq \frac{\Delta}{\bar{n}_{B}} n_{A A} \Sigma_{a A, a B}-n_{a A}\left(\Delta-\eta n_{B B}\right)+\Theta\left(\Delta^{2} n_{A A}\right) .
$$

Using that $\eta<c$, the slope of this process if $n_{a A}$ reaches the lower bound is estimated by

$$
\begin{aligned}
\dot{\Sigma}_{a A, a B} & \leq \frac{\Delta}{\bar{n}_{B}} n_{A A} \Sigma_{a A, a B}-\frac{f\left(2 n_{A A}+n_{A B}\right)}{4 \bar{n}_{B}(f+\Delta)}\left(\Delta-\eta n_{B B}\right) \Sigma_{a A, a B}+\Theta\left(\Delta^{2} n_{A A}\right) \\
& \leq \frac{f\left(2 n_{A A}+n_{A B}\right)}{4-D} \frac{f-D}{f+\Delta} \Sigma_{a A, a B}+\frac{\Delta}{\bar{n}_{B}} n_{A A} \Sigma_{a A, a B}+\Theta\left(\Delta^{2} n_{A A}\right) .
\end{aligned}
$$

Moreover we need majorising processes on $A A$ and $A B$ :

$$
\begin{aligned}
& b_{A B} \leq \frac{f}{\Sigma_{5}} n_{A B}\left(\frac{1}{2} n_{A B}+n_{A A}+n_{B B}\right)+\frac{2 f}{\Sigma_{5}} n_{A A} n_{B B}+\Theta(\delta), \\
& d_{A B} \geq n_{A B}\left(f-\frac{\Delta(1+\Delta)}{\bar{n}_{B}} n_{A A}\right), \\
& \dot{n}_{A B} \leq-\frac{f}{2 \Sigma_{5}} n_{A B}^{2}+\frac{2 f}{\Sigma_{5}} n_{A A} n_{B B}+\frac{\Delta(1+\Delta)}{\bar{n}_{B}} n_{A A} n_{A B}+\Theta(\delta), \\
& b_{A A} \leq \frac{f}{\Sigma_{5}} n_{A A}\left(n_{A B}+n_{A A}\right)+\frac{f}{4 \Sigma_{5}} n_{A B}^{2}+\Theta(\delta), \\
& d_{A A} \geq n_{A A}\left(f+\Delta-\frac{\Delta(1+\Delta)}{\bar{n}_{B}} n_{A A}\right), \\
& \dot{n}_{A A} \geq-n_{A A}\left(\frac{f}{\Sigma_{5}} n_{B B}+\Delta-\frac{\Delta(1+\Delta)}{\bar{n}_{B}} n_{A A}\right)+\frac{f}{4 \Sigma_{5}} n_{A B}^{2}+\Theta(\delta) .
\end{aligned}
$$

Thus we have

$$
\dot{n}_{A B}+2 \dot{n}_{A A} \leq-\Delta n_{A A}\left(2-\frac{2 n_{A A}+n_{A B}}{\bar{n}_{B}}\right)+\Theta\left(\Delta^{2} n_{A A}\right)<\Theta\left(\Delta^{2} n_{A A}\right) .
$$

It is enough to show that

$$
\dot{n}_{a A} \geq \frac{f\left(2 n_{A A}+n_{A B}\right)}{4 \bar{n}_{B}(f+\Delta)} \dot{\Sigma}_{a A, a B}+\Theta\left(\Delta^{2} n_{A A}\right) \Sigma_{a A, a B}
$$


using that $\eta<c$ we have

$$
\begin{aligned}
& \frac{f\left(2 n_{A A}+n_{A B}-\Theta(\delta)\right)}{4 \bar{n}_{B}} \Sigma_{a A, a B}-\frac{f^{2}\left(n_{A B}+2 n_{A A}\right)^{2}}{16 \bar{n}_{B}^{2}(f+\Delta)} \frac{f-D}{f+\Delta} \Sigma_{a A, a B} \\
& -\frac{f\left(n_{A B}+2 n_{A A}\right)}{4 \bar{n}_{B}(f+\Delta)} \frac{\Delta}{\bar{n}_{B}} n_{A A} \Sigma_{a A, a B}-\Theta\left(\Delta^{2} n_{A A}\right) \Sigma_{a A, a B} \\
& \geq \frac{f\left(2 n_{A A}+n_{A B}-\Theta(\delta)\right)}{4 \bar{n}_{B}} \Sigma_{a A, a B}-\frac{f\left(2 n_{A A}+n_{A B}\right)}{8 \bar{n}_{B}} \Sigma_{a A, a B}\left(1+\frac{2 \Delta(1+\Delta) n_{A A}}{\bar{n}_{B}(f+\Delta)}\right)-\Theta\left(\Delta^{2} n_{A A}\right) \Sigma_{a A, a B}
\end{aligned}
$$

This finishes the proof.

Proposition 4.16. For all $t \in\left[T_{1}, T_{2}\right]$ the process $\Sigma_{a A, a B}$ is bounded by

(1) $\dot{\Sigma}_{a A, a B} \leq n_{a A}\left(\eta n_{B B}-\Delta \frac{n_{A B}+\Theta\left(\Delta n_{A A}\right)}{n_{A B}+2 n_{A A}}\right)$.

(2) $\dot{\Sigma}_{a A, a B} \geq n_{a A}\left(\eta n_{B B}-\Delta-\Theta(\delta)\right)$.

Proof.

(1) We construct a majorising process on $\Sigma_{a A, a B}$ and use Proposition 4.6 and Lemma 4.8 .

$$
\begin{aligned}
b_{\Sigma_{a A, a B}} & \leq n_{a A} \frac{f\left(\frac{1}{2} n_{a A}+n_{A A}+n_{a B}+n_{A B}+n_{B B}\right)}{\Sigma_{5}}+n_{a B} \frac{f\left(n_{A A}+\frac{1}{2} n_{a B}+n_{A B}+n_{B B}\right)}{\Sigma_{5}}+\Theta\left(\Sigma_{a A, a B}^{2}\right) \\
& \leq f \Sigma_{a A, a B}+\Theta\left(\Sigma_{a A, a B}^{2}\right), \\
d_{\Sigma_{a A, a B}} & \geq \Sigma_{a A, a B}\left(D-\Delta+c \Sigma_{5}\right)+\Delta n_{a A}-\eta n_{a A} n_{B B} \\
& \geq \Sigma_{a A, a B}\left(f-\frac{\Delta(1+\Delta)}{\bar{n}_{B}} n_{A A}\right)+\Delta n_{a A}-\eta n_{a A} n_{B B}, \\
\dot{\Sigma}_{a A, a B} & \leq \frac{\Delta(1+\Delta)}{\bar{n}_{B}} n_{A A} n_{a B}-n_{a A}\left(\Delta-\frac{\Delta(1+\Delta)}{\bar{n}_{B}} n_{A A}-\eta n_{B B}\right)+\Theta\left(\Sigma_{a A, a B}^{2}\right) .
\end{aligned}
$$

To bound $n_{a B}$ we use Lemma 4.10 .

$$
\begin{aligned}
\dot{\Sigma}_{a A, a B} & \leq n_{a A}\left(\frac{\Delta(1+\Delta) n_{A A}}{n_{A B}+2 n_{A A}} \frac{n_{A B}+2 n_{B B}+\frac{2 \Delta}{c}}{\bar{n}_{B}}-\Delta+\frac{\Delta(1+\Delta)}{\bar{n}_{B}} n_{A A}+\eta n_{B B}+\Theta(\delta)\right), \\
& \leq n_{a A}\left(\eta n_{B B}+\frac{\Delta\left(n_{A A}\left(n_{A B}+2 n_{B B}\right)+n_{A A}\left(n_{A B}+2 n_{A A}\right)-\bar{n}_{B}\left(n_{A B}+2 n_{A A}\right)\right)+\Theta\left(\Delta^{2} n_{A A}\right)}{\bar{n}_{B}\left(n_{A B}+2 n_{A A}\right)}\right) \\
& \leq n_{a A}\left(\eta n_{B B}-\Delta \frac{n_{A B}+\Theta\left(\Delta n_{A A}\right)}{n_{A B}+2 n_{A A}}\right) .
\end{aligned}
$$

(2) This time we construct a minorising process on $\Sigma_{a A, a B}$ by using Proposition 4.6 and Lemma 4.8.

$$
\begin{aligned}
b_{\Sigma_{a A, a B}} & \geq f \frac{n_{a A}\left(\frac{1}{2} n_{a A}+n_{A A}+n_{a B}+n_{A B}+n_{B B}\right)+n_{a B}\left(n_{A A}+\frac{1}{2} n_{a B}+n_{A B}+n_{B B}\right)}{n_{a A}+n_{A A}+n_{a B}+n_{A B}+n_{B B}}-\Theta\left(\delta^{2}\right) \\
& \geq f \Sigma_{a A, a B}-\Theta\left(\delta^{2}\right) \\
d_{\Sigma_{a A, a B}} & \leq \Sigma_{a A, a B}\left(D-\Delta+c \Sigma_{5}\right)-\eta n_{a A} n_{B B}+\left(\Delta+\Theta\left(\delta^{2}\right) n_{a A}\right. \\
& \leq f \Sigma_{a A, a B}-n_{a A}\left(\eta n_{B B}-\Delta-\Theta\left(\delta^{2}\right)\right) \\
\dot{\Sigma}_{a A, a B} & \geq n_{a A}\left(\eta n_{B B}-\Delta-\Theta(\delta)\right) .
\end{aligned}
$$


From this Proposition we can deduce

Corollary 4.17. There exists a $t^{*} \in\left[T_{1}, T_{2}\right]$, such that for all $t \in\left[t^{*}, T_{2}\right]$ and $\eta>\frac{4 \Delta}{\bar{n}_{B}}=: \eta^{\star}$, it holds

$$
\dot{\Sigma}_{a A, a B}(t)>0 .
$$

Proof. A fine calculation will show that the competition $c-\eta$ felt by an $a A$-individual from a $B B$-individual allow the sum $\Sigma_{a A, a B}$ to grow when $\eta$ is large enough, whereas it decreases when $\eta=0$. Note that we consider here the sum $\Sigma_{a A, a B}$ because the influence of $\eta$ cannot be seen in the rates of the $a B$-population alone. Heuristically, the growth of the $a B$-population happens due to the indirect influence (source of $a$-allele) of the less decaying $a A$-population. We prove that the minorising process on $\Sigma_{a A, a B}$ estimated in the Proposition 4.16 starts to increase:

$$
\dot{\Sigma}_{a A, a B} \geq n_{a A}\left(\eta n_{B B}-\Delta-\Theta(\delta)\right) .
$$

As soon as $n_{B B}>\Delta / \eta$, the sum-process $\Sigma_{a A, a B}$ starts to increase. From Lemma 4.11 and Proposition 4.13 we know that, for $t \geq T_{=}$, we have $n_{B B} \geq \frac{\bar{n}_{B}}{4}-\Theta(\Delta)$. Hence, if we choose $\eta>\frac{4 \Delta}{\bar{n}_{B}}$ the sum-process $\Sigma_{a A, a B}$ increases.

Now we are able to calculate the time $T^{a A=\delta A A} \wedge T^{a B=\delta A B}$ and we will see that $T^{a A=\delta A A} \wedge$ $T^{a B=\delta A B}=T^{a A=\delta A A}$.

Theorem 4.18. The time $T_{2}=\Theta\left(\varepsilon^{-1 /\left(1+\eta \bar{n}_{B}-\Delta\right)}\right)$.

Proof. From Proposition 4.16 (2) we have a lower bound on $\dot{\Sigma}_{a A, a B}$, and with Lemma 4.15 (2) we can bound this further from below by:

$$
\begin{aligned}
\dot{\Sigma}_{a A, a B} & \geq n_{a A}\left(\eta n_{B B}-\Delta-\Theta(\delta)\right) \\
& \geq\left(\eta n_{B B}(t)-\Delta-\Theta(\delta)\right) \frac{f\left(n_{A B}(t)+2 n_{A A}(t)\right)}{4 \bar{n}_{B}(f+\Delta)} \Sigma_{a A, a B}(t) \\
& \geq \frac{\Theta\left(\eta \bar{n}_{B} / 4-\Delta\right)}{\Theta(1)+\Theta(1) t} \Sigma_{a A, a B}(t) .
\end{aligned}
$$

where the last estimation on $n_{B B}$ and on $n_{A B}$ comes from Proposition 4.13 and from [25] since we know from there that the time until $n_{A B}=\Theta\left(\sqrt{n_{A A}}\right)$, starts to decrease like $1 / t$ is of order $\Theta(1)$. As $\Sigma_{a A, a B}\left(T_{1}\right)=\Theta(\varepsilon)$, the solution of the lower-bounding ODE is:

$$
\Sigma_{a A, a B}(t) \geq \Theta(\varepsilon)(\Theta(1)+\Theta(1) t)^{\Theta\left(\eta \bar{n}_{B} / 4-\Delta\right)}
$$

By using Proposition 4.16 (1), we get the same kind of solution as an upper bound on $\Sigma_{a A, a B}$ (note on the last step we can upper bound $n_{B B}$ by $\bar{n}_{B}$ ):

$$
\Sigma_{a A, a B}(t) \leq \Theta(\varepsilon)(\Theta(1)+\Theta(1) t)^{\Theta\left(\eta \bar{n}_{B}-\Delta\right)}
$$

Using (4.230) and Lemma 4.15 we get a minorising process on $a A$ :

$$
n_{a A}(t)=\Theta\left(n_{A B} \Sigma_{a A, a B}\right) \geq \Theta(\varepsilon)(\Theta(1)+\Theta(1) t)^{\Theta\left(\eta \bar{n}_{B} / 4-\Delta\right)} /(\Theta(1)+\Theta(1) t) .
$$

The corresponding majorising process has an $\bar{n}_{B}$ instead of $\bar{n}_{B} / 4$. By solving $n_{a A}=\delta n_{A A}=$ $\Theta\left(n_{A B}^{2}\right)$ we get the order of magnitude of $T_{a a=\delta A A}$ :

$$
\Theta\left(\varepsilon^{-1 /\left(1+\eta \bar{n}_{B}-\Delta\right)}\right) \leq T_{a a=\delta A A} \leq \Theta\left(\varepsilon^{-1 /\left(1+\eta \bar{n}_{B} / 4-\Delta\right)}\right)
$$

Note that $1+\eta \bar{n}_{B}-\Delta>0$ for $\Delta$ small enough, and thus $T_{a a=\delta A A}$ diverges with $\varepsilon$ and the order calculations above are justified. 
It is left to ensure that $a B$ does not exceed $\delta n_{A B}$ in this time. It follows from Lemma 4.15 that during the time interval $\left[T_{1}, T_{2}\right]$, we have $\Sigma_{a A, a B}=\Theta\left(n_{a B}\right)$. Thus, solving $n_{a B}=\delta n_{A B}$ amounts to solving $\Theta\left(\Sigma_{a A, a B}\right)=\Theta(1) /(\Theta(1)+\Theta(1) t)$ which gives the very same order of magnitude as for $T_{a A=\delta A A}$. Thus the two times are of the same order.

Note that for $\eta=0, \Sigma_{a A, a B}\left(T_{2}\right)=\Theta\left(\varepsilon^{1+\Delta /(1-\Delta)}\right)$. This proves point 1 of Theorem 3.1

Proposition 4.19. $T_{2}=T^{a A=\delta A A}$

Proof. This follows from Theorem 4.18 and Proposition 4.8 .

Proposition 4.20. At time $t=T_{2}$ and if $f$ is taken sufficiently large (Assumption C2), $n_{a a}$ starts to grow out of itself: there exists some positive constant $c_{T_{2}}>0$ such that

$$
\dot{n}_{a a} \geq c_{T_{2}} \cdot n_{a a} .
$$

Proof. We have $n_{A A}\left(T_{2}\right)=\Theta\left(\varepsilon^{2 /\left(1+\eta \bar{n}_{B}-\Delta\right)}\right)$. Thus, at the end of the second phase,

$$
\begin{aligned}
& b_{a a} \geq f n_{a a} \frac{\frac{1}{2} \delta n_{A A}}{n_{A A}(1+\Theta(\delta))}=\frac{\delta f n_{a a}}{2(1+\Theta(\delta))}, \\
& d_{a a} \leq n_{a a}\left(D+\Delta+n_{A A}(1+\Theta(\delta))\right)=n_{a a}\left(D+\Delta+\Theta\left(\varepsilon^{2 /\left(1+\eta \bar{n}_{B}-\Delta\right)}\right),\right. \\
& \dot{n}_{a a} \geq n_{a a}\left(\frac{\delta f}{2}-D-\Delta-\Theta\left(\varepsilon^{2 /\left(1+\eta \bar{n}_{B}-\Delta\right)}\right)\right) .
\end{aligned}
$$

the right-hand side is positive for $f$ large enough.

4.4. Phase 3: Exponential growth of $a a$ until co-equilibrium with $B B$. Since $a a$ is growing now also out of itself it will influence the sum-process $\Sigma_{5}=n_{a A}+n_{A A}+n_{a B}+n_{A B}+$ $n_{B B}$ and we need new lower bounds on $\Sigma_{5}$ in the following steps, the proof of this works similar to the one of Proposition 4.6 by taking into account all contributing populations. Let us compute the ODE to which $\Sigma_{5}$ is the solution:

Proposition 4.21. The sum-process $\Sigma_{5}$ is the solution to

$$
\begin{aligned}
\dot{\Sigma}_{5}= & \Sigma_{5}\left(f-D-\Delta-c \Sigma_{5}\right)-\Delta\left(n_{a A}+n_{A A}\right)-c n_{a a}\left(n_{a A}+n_{A A}\right)+2 \eta n_{a A} n_{B B} \\
& +\frac{f}{\Sigma_{3}} n_{a a}\left(\frac{1}{2} n_{a A}+n_{A A}\right)-\frac{f}{4 \Sigma_{5}} n_{a B}\left(n_{a A}+n_{a B}\right)-\frac{f}{4 \Sigma_{6}} n_{a A}\left(2 n_{a a}+n_{a A}+n_{a B}\right) .
\end{aligned}
$$

Proof. We calculate the birth- and the death-rate of $\Sigma_{5}$ under consideration of the $a a$ population:

$$
b_{\Sigma_{5}}=\frac{f}{\Sigma_{3}} n_{a a}\left(\frac{1}{2} n_{a A}+n_{A A}\right)+\frac{f}{\Sigma_{5}}\left(\left(n_{a B}+n_{A B}+n_{B B}\right) \Sigma_{5}-\frac{1}{4} n_{a B}\left(n_{a A}+n_{a B}\right)\right)
$$
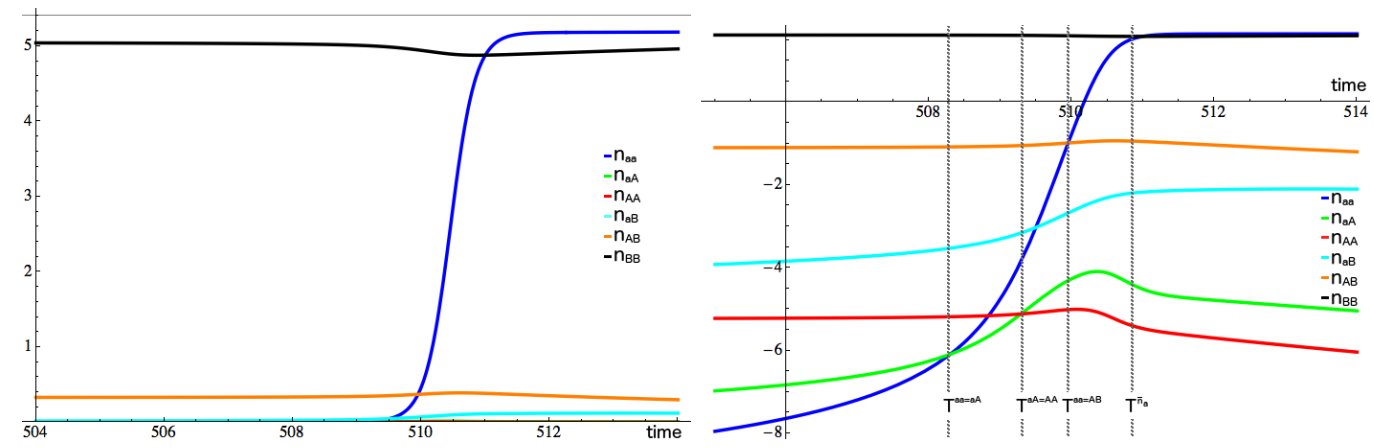

FIGURE 6. zoom-in when aa recovers, general qualitative behaviour of $\left\{n_{i}(t), i \in \mathcal{G}\right\}$ (lhs) and log-plot (rhs). 


$$
\begin{aligned}
& +\frac{f}{\Sigma_{6}}\left(\left(n_{a A}+n_{A A}\right) \Sigma_{6}-n_{a A}\left(\frac{1}{2} n_{a a}+\frac{1}{4} n_{a A}+\frac{1}{4} n_{a B}\right)\right) \\
= & f \Sigma_{5}+\frac{f}{\Sigma_{3}} n_{a a}\left(\frac{1}{2} n_{a A}+n_{A A}\right)-\frac{f}{4 \Sigma_{5}} n_{a B}\left(n_{a A}+n_{a B}\right)-\frac{f}{4 \Sigma_{6}} n_{a A}\left(2 n_{a a}+n_{a A}+n_{a B}\right), \\
d_{\Sigma_{5}}= & \Sigma_{5}\left(D-\Delta+c \Sigma_{5}\right)+\left(c n_{a a}+\Delta\right)\left(n_{a A}+n_{A A}\right)-2 \eta n_{a A} n_{B B}, \\
\dot{\Sigma}_{5}= & \Sigma_{5}\left(f-D-\Delta-c \Sigma_{5}\right)-\left(c n_{a a}+\Delta\right)\left(n_{a A}+n_{A A}\right)+2 \eta n_{a A} n_{B B} \\
& +\frac{f}{\Sigma_{3}} n_{a a}\left(\frac{1}{2} n_{a A}+n_{A A}\right)-\frac{f}{4 \Sigma_{5}} n_{a B}\left(n_{a A}+n_{a B}\right)-\frac{f}{4 \Sigma_{6}} n_{a A}\left(2 n_{a a}+n_{a A}+n_{a B}\right) .
\end{aligned}
$$

Let

$$
T_{3}:=T_{\bar{n}_{a}-\varepsilon^{\gamma / 2}}^{a a}=\inf \left\{t>T_{2}: n_{a a}(t)=\bar{n}_{a}-\varepsilon^{\gamma / 2}\right\} .
$$

We will need some preliminary bounds on $n_{a A}$ and $n_{A A}$.

Lemma 4.22. For all $t \in\left[T_{2}, T_{3}\right]$,

$$
\begin{aligned}
& n_{a A} \leq \Theta\left(\max \left\{n_{a a}, n_{a A}, n_{a B}\right\} n_{A B}\right) \leq \Theta\left(n_{A B}\right), \\
& n_{A A} \leq \Theta\left(\max \left\{n_{a A}, n_{A A}, n_{A B}\right\}^{2}\right) \leq \Theta\left(n_{A B}\right) .
\end{aligned}
$$

Proof. The populations $a A$ and $A A$ always stays smaller than or equal to $\Theta\left(n_{A B}\right)$ since they are produced in majority from recombination of $A B$ with other smaller population.

We divide this phase into steps (see Figure 6):

Step 1: $\left[T_{2}, T^{a a=a A}\right]$,

Step 2: $\left[T^{a a=a A}, T^{a a=A B}\right]$,

Step 3: $\left[T^{a a=A B}, T_{3}\right]$.

We distinguish two cases in Step $1\left(T^{a A=A A} \geq T^{a a=a A}\right.$ and $\left.T^{a A=A A} \leq T^{a a=a A}\right)$, as well as in Step $2\left(T^{a A=A A} \geq T^{a a=A B}\right.$ and $\left.T^{a A=A A} \leq T^{a a=A B}\right)$ since we cannot prove which one happens in general. We introduce some notation for the order of magnitude of $n_{A A}\left(T_{2}\right)$. We write $n_{A A}\left(T_{2}\right)=\Theta\left(\varepsilon^{\gamma}\right)$ with

$$
\gamma:=2 /\left(1+\eta \bar{n}_{B}-\Delta\right) .
$$

\subsubsection{Step 1: Time interval $\left[T_{2}, T^{a a=a A}\right]$.}

Proposition 4.23. For all $\eta<\frac{c}{2}$, we have $T^{a a=a A}<\infty$ and for all $t \in\left[T_{2}, T^{a a=a A}\right]$ it holds

- the aa-population grows exponentially fast,

- $n_{A A}\left(T^{a a=a A}\right)=\Theta\left(\varepsilon^{\gamma}\right)$,

- $n_{A B}\left(T^{a a=a A}\right)=\Theta\left(\varepsilon^{\gamma / 2}\right)$.

Proof. In this step we show that aa crosses the $a A$-population.

(1) Case 1: $T^{a A=A A} \geq T^{a a=a A}$.

In this case $n_{a a} \leq n_{a A} \leq n_{A A}$. First note that the birth-rate of $a A, b_{a A}$, gets an additional contributing term, namely:

$$
f n_{a a} \frac{\frac{1}{2} n_{a A}+n_{A A}}{n_{a a}+n_{a A}+n_{A A}} \leq f n_{a a} \frac{\frac{3}{2} \max \left\{n_{a A}, n_{A A}\right\}}{\max \left\{n_{a A}, n_{A A}\right\}}=\frac{3}{2} f n_{a a} .
$$

Since $n_{a A}=\Theta\left(n_{a B} n_{A B}\right), n_{a a} \leq n_{a A}$ and $n_{a B} \leq n_{A B}$ (cf. Proposition 4.3) in this step the main contribution to the $a A$-population still comes from matchings of $a B$ and $A B$-individual and thus $a A$ increases but stays of the same order. Considering the birth-rate $b_{a a}$, we see that only a growing $A A$-population could stop the growth of $a a$. Thus we have to ensure that this population stays small enough. 
Lemma 4.24. The AA-population is bounded from above by

$$
n_{A A} \leq \frac{1}{\bar{n}_{A}} n_{A B}^{2} .
$$

Proof. Looking at the rates of $A A$ we see that an increasing $a a$-population has less influence on the growth of $A A$ since it only raises the pool of possible partners and increases the competition (cf. Theorem 4.5). Thus the $a a$-population directly can only lower the growth of $A A$ and since $a A$ is always smaller than $A B$, the $A A$-population behaves like $n_{A B}^{2}$ as before. More precisely,

$$
\begin{aligned}
& b_{A A} \leq \frac{f}{\Sigma_{5}}\left(\frac{1}{2} n_{a A}+n_{A A}+\frac{1}{2} n_{A B}\right)^{2}=\frac{f}{\Sigma_{5}} n_{A A}\left(n_{a A}+n_{A A}+n_{A B}\right)+\frac{f}{4 \Sigma_{5}}\left(n_{a A}+n_{A B}\right)^{2}, \\
& b_{A A} \geq \frac{f}{\Sigma_{5}} n_{A A}\left(n_{a A}+n_{A A}+n_{A B}\right)+\frac{f}{4 \Sigma_{5}}\left(n_{a A}+n_{A B}\right)^{2}-\frac{3 f}{\Sigma_{6} \Sigma_{5}} n_{a a} n_{A A} n_{A B}, \\
& d_{A A} \geq n_{A A}(f-\Theta(\Delta)), \\
& \left.d_{A A} \leq n_{A A}\left(f+\Theta(\Delta)+c n_{a a}\right)\right), \\
& \dot{n}_{A A} \leq-n_{A A}\left(\frac{f}{\Sigma_{5}}\left(n_{B B}+n_{a B}\right)-\Theta(\Delta)\right)+\frac{f}{4 \Sigma_{5}}\left(n_{A B}^{2}+2 n_{A A} n_{A B}+n_{A A}^{2}\right), \\
& \dot{n}_{A A} \geq-n_{A A}\left(\frac{f}{\Sigma_{5}}\left(n_{B B}+n_{a B}\right)+\Theta(\Delta)\right)+\frac{f}{4 \Sigma_{5}}\left(n_{A B}^{2}+2 n_{A A} n_{A B}+n_{A A}^{2}\right) .
\end{aligned}
$$

We use again Lemma 4.2. The majorising process on $A A$ at the upper bound decreases

$$
\dot{n}_{A A} \leq-\frac{3 f(1+\Theta(\Delta))}{4 \Sigma_{5}} n_{A B}^{2} .
$$

It is left to show that $\dot{n}_{A A} \leq \frac{2}{\bar{n}_{A}} n_{A B} \dot{n}_{A B}$. For this we estimate a minorising process on $A B$ :

$$
\begin{aligned}
b_{A B} & \geq \frac{2 f}{\Sigma_{6}}\left(\frac{1}{2} n_{a A}+n_{A A}+\frac{1}{2} n_{A B}\right)\left(\frac{1}{2} n_{a B}+\frac{1}{2} n_{A B}+n_{B B}\right) \\
& \geq f n_{A B}-\frac{f}{\Sigma_{6}} n_{A B}\left(n_{a a}+\frac{1}{2} n_{a A}+\frac{1}{2} n_{a B}+\frac{1}{2} n_{A B}\right)+\frac{2 f}{\Sigma_{6}} n_{A A}\left(\frac{1}{2} n_{a B}+n_{B B}\right), \\
d_{A B} & \leq n_{A B}\left(f+\Theta\left(n_{a A}\right)\right), \\
\dot{n}_{A B} & \geq-\frac{f}{\Sigma_{6}} n_{A B}\left(n_{A B}+\Theta\left(n_{a A}\right)\right)+\frac{2 f}{\Sigma_{6}} n_{A A}\left(\frac{1}{2} n_{a B}+n_{B B}\right) .
\end{aligned}
$$

At the upper bound the process would increase $\dot{n}_{A B} \geq \frac{f(1-\Theta(\Delta))}{\Sigma_{6}} n_{A B}^{2}$ and hence the upper bound holds.

We now have to find a majorising process on $A B$.

Lemma 4.25. For the AB-population it holds:

$$
\dot{n}_{A B} \leq \Theta\left(n_{A B}^{2}\right)
$$

Proof.

$$
\begin{aligned}
b_{A B} & \leq \frac{2 f}{\Sigma_{5}}\left(\frac{1}{2} n_{a A}+n_{A A}+\frac{1}{2} n_{A B}\right)\left(\frac{1}{2} n_{a B}+\frac{1}{2} n_{A B}+n_{B B}\right) \\
& =\frac{f}{\Sigma_{5}} n_{A B}\left(\frac{1}{2} n_{a A}+n_{A A}+\frac{1}{2} n_{a B}+\frac{1}{2} n_{A B}+n_{B B}\right)+\frac{2 f}{\Sigma_{5}}\left(\frac{1}{2} n_{a A}+n_{A A}\right)\left(\frac{1}{2} n_{a B}+n_{B B}\right), \\
d_{A B} & \geq n_{A B}\left(f-\Theta\left(\Delta n_{A B}\right)\right), \\
\dot{n}_{A B} & \leq-n_{A B}\left(\frac{f}{2 \Sigma_{5}}\left(n_{a A}+n_{a B}+n_{A B}\right)-\Theta\left(\Delta n_{A B}\right)\right)+\frac{3 f}{\Sigma_{5}} n_{A A}\left(\frac{1}{2} n_{a B}+n_{B B}\right) \leq \Theta\left(n_{A B}^{2}\right),
\end{aligned}
$$

by Lemma 4.24 Thus

$$
n_{A B}(t) \leq \frac{\Theta(1)}{\Theta\left(\varepsilon^{-\gamma / 2}\right)-\Theta(1) t} .
$$


The time $T_{\varepsilon^{\gamma / 2}}^{A B}$ the $A B$-population needs to exceed $\Theta\left(\varepsilon^{\gamma / 2}\right)$ is $T_{\varepsilon^{\gamma / 2}}^{A B} \geq \Theta\left(\varepsilon^{-\gamma / 2}\right)$. Since $n_{A A}(t) \leq \Theta\left(n_{A B}^{2}\right)$, the time $T_{\varepsilon^{\gamma}}^{A A}$ the $A A$-population needs to exceed the order $\Theta\left(\varepsilon^{\gamma}\right)$ is $T_{\varepsilon^{\gamma}}^{A A} \geq \Theta\left(\varepsilon^{-\gamma / 2}\right)$. Until time $T_{\varepsilon^{\gamma}}^{A A}$ we consider a minorising process on $a a$ :

$$
\begin{aligned}
& b_{a a} \geq f n_{a a} \frac{\Theta(\delta) n_{A A}}{\Theta(1) n_{A A}}=\Theta(\delta) f n_{a a}, \\
& d_{a a} \leq n_{a a}\left(D+\Delta+\Theta(1) n_{A A}\right), \\
& \dot{n}_{a a} \geq n_{a a}\left(\Theta(\delta) f-D-\Delta-\Theta(1) n_{A A}\right),
\end{aligned}
$$

Using Lemma 4.95 we get

$$
n_{a a}(t) \geq \Theta\left(\varepsilon_{0}^{2}\right) e^{\Theta(\delta) t} .
$$

Thus $a a$ grows exponentially fast and reaches $\Theta\left(\varepsilon^{\gamma}\right)$ in time $\Theta\left(\ln \left(\varepsilon^{-\gamma}\right)\right)$. This time is shorter than $T_{\varepsilon^{\gamma}}^{A A}$ and we are done.

(2) Case 2: $T^{a A=A A} \leq T^{a a=a A}$.

In this case $n_{A A} \leq n_{a A}$ and $n_{a a} \leq n_{a A}$. The $a A$-population has the same additional term in ist birth rate as in the case before and by the same reasoning $n_{a A}$ stays smaller than $\Theta\left(n_{A B}^{2}\right)$. We make this more precise by considering the growth of $A B$ and calculating upper bounds on $a A$ and $A A$ :

Lemma 4.26. The AA-population is bounded from above by

$$
n_{A A} \leq \frac{2}{\bar{n}_{A}} n_{A B}^{2} .
$$

Proof. The proof is similar to the one of Lemma 4.8. We have to show that at the upper bound $\dot{n}_{A A} \leq \frac{4}{\bar{n}_{A}} \dot{n}_{A B} n_{A B}$. Observe that the lower bound on $\Sigma_{5}$ also holds here. We start by estimating a majorising process on $A A$ and by calculating the slope of it at the upper bound:

$$
\begin{aligned}
b_{A A} & \leq \frac{f}{\Sigma_{5}}\left(\frac{1}{2} n_{a A}+n_{A A}+\frac{1}{2} n_{A B}\right)^{2} \\
& =\frac{f}{\Sigma_{5}} n_{A A}\left(n_{a A}+n_{A A}+n_{A B}\right)+\frac{f}{4 \Sigma_{5}}\left(n_{a A}+n_{A B}\right)^{2}, \\
d_{A A} & \geq n_{A A}(f-\Theta(\Delta)), \\
\dot{n}_{A A} & \leq-n_{A A}(f-\Theta(\Delta))+\frac{f}{\Sigma_{5}} n_{A B}^{2} .
\end{aligned}
$$

The slope of this majorising process at the upper bound is estimated by $\dot{n}_{A A} \leq$ $-\frac{f-\Theta(\Delta)}{\bar{n}_{A}} n_{A B}^{2}<0$. Thus if we can show that the slope of a minorising process on $A B$ is positive at the upper bound we are done:

$$
\begin{aligned}
& b_{A B} \geq f n_{A B}-\frac{f}{2 \Sigma_{6}} n_{A B}\left(2 n_{a a}+n_{a A}+n_{a B}+n_{A B}\right)+\frac{2 f}{\Sigma_{6}}\left(\frac{1}{2} n_{a A}+n_{A A}\right)\left(\frac{1}{2} n_{a B}+n_{B B}\right), \\
& d_{A B} \leq n_{A B}\left(f+\Theta\left(\Delta n_{A B}\right)\right), \\
& \dot{n}_{A B} \geq-\frac{3 f+\Theta(\Delta)}{\Sigma_{6}} n_{A B}^{2}+\frac{3 f}{\Sigma_{6}} n_{A A}\left(\frac{1}{2} n_{a B}+n_{B B}\right) .
\end{aligned}
$$

The slope of this process at the upper bound can be estimated by $\dot{n}_{A B} \geq \frac{3 f-\Theta(\Delta)}{2 \bar{n}_{B}} n_{A B}^{2}>$ 0 . This finishes the proof.

We proceed similarly for the upper bound on $a A$ :

Lemma 4.27. For $\eta<\frac{c}{2}$, the aA-population is bounded from above by

$$
n_{a A} \leq \frac{6 f}{\bar{n}_{A}(D-\Delta)} n_{A B}^{2}
$$


Proof. This time we have to show that at the upper bound $\dot{n}_{a A} \leq \frac{12 f}{\bar{n}_{A}(D-\Delta)} \dot{n}_{A B} n_{A B}$. Using Proposition 4.21, Lemma 4.29 and $\eta<\frac{c}{2}$ we estimate a majorising process on $a A$ and calculate the slope of it at the upper bound:

$$
\begin{aligned}
b_{a A} \leq & \frac{f}{2 \Sigma_{3}} n_{a a} n_{a A}+\frac{f}{\Sigma_{3}} n_{a a} n_{A A}+\frac{f}{2 \Sigma_{5}} n_{a A}\left(n_{a A}+2 n_{A A}+n_{a B}+n_{A B}\right) \\
& +\frac{f}{2 \Sigma_{5}} n_{a B}\left(2 n_{A A}+n_{A B}\right)+\frac{f}{\Sigma_{5}} n_{a a}\left(\frac{1}{2} n_{a A}+n_{A A}\right), \\
d_{a A} \geq & n_{a A}\left(f-\Theta(\Delta)-\eta n_{B B}\right) \geq \frac{f+D-\Theta(\Delta)}{2} n_{a A}, \\
\dot{n}_{a A} \leq & -\frac{D-\Theta(\Delta)}{2} n_{a A}+\frac{5 f+\Theta(\Delta)}{2 \bar{n}_{A}} n_{A B}^{2} .
\end{aligned}
$$

The slope of this majorising process at the upper bound is estimated by $\dot{n}_{a A} \leq$ $-\frac{f-\Theta(\Delta)}{2 \bar{n}_{A}} n_{A B}^{2}<0$. Thus if we can show that the slope of a minorising process on $A B$ would be positive at the upper bound we are done. For this we use the minorising process of $A B$ from before (cf.(4.274)) and estimate the slope which is given by $\dot{n}_{A B} \geq \frac{3 f-\Theta(\Delta)}{2 \bar{n}_{B}} n_{A B}^{2}>0$. This finishes the proof.

Now we can estimate a majorising process on $A B$ and can bound it by $\dot{n}_{A B} \leq$ $-\Theta\left(n_{A B}^{2}\right)$, hence $n_{A B}(t) \leq \frac{\Theta(1)}{\Theta\left(\varepsilon^{-\gamma / 2}\right)-\Theta(1) t}$. We have to construct a minorising process on $a a$. Since now $n_{A A} \leq n_{a A}$ and $n_{a a} \leq n_{a A}$ we can estimate:

$$
\begin{aligned}
& b_{a a} \geq f n_{a a} \frac{\frac{1}{2} n_{a A}}{3 n_{a A}}=\frac{f}{6} n_{a a}, \\
& d_{a a} \leq n_{a a}\left(D+\Delta+\Theta\left(n_{A B}^{2}\right)\right), \\
& \dot{n}_{a a} \geq n_{a a}\left(\frac{f}{6}-D-\Theta\left(\Delta^{2}\right)\right) .
\end{aligned}
$$

Thus the aa-population grows exponentially fast $n_{a a}(t) \geq \Theta\left(\varepsilon^{2 \gamma}\right) e^{\left(f / 6-D-\Theta\left(\Delta^{2}\right)\right) t}$. The time $T_{\varepsilon^{\gamma / 2}}^{A B}$ the $A B$-population needs to exceed $\Theta\left(\varepsilon^{\gamma / 2}\right)$, is $T_{\varepsilon^{\gamma / 2}}^{A B}=\Theta\left(\varepsilon^{-\gamma / 2}\right)$. Since $n_{a A} \leq \Theta\left(n_{A B}^{2}\right)$ until this time it holds $n_{a A} \leq \Theta\left(\varepsilon^{\gamma}\right)$. The time $T_{\varepsilon^{\gamma}}^{a a}$, aa needs to reach $\Theta\left(\varepsilon^{\gamma}\right)$ is $T_{\varepsilon^{\gamma}}^{a a}=\Theta\left(\ln \varepsilon^{-\gamma}\right)$. Thus $T_{\varepsilon^{\gamma}}^{a a} \leq T_{\varepsilon^{\gamma / 2}}^{A B}$ and $T^{a a=a A}<\infty$.

\subsubsection{Step 2: Time interval $\left[T^{a a=a A}, T^{a a=A B}\right]$.}

Proposition 4.28. For $\eta<\frac{c}{2}$, we have $T^{a a=A B}<\infty$ and for all $t \in\left[T^{a a=a A}, T^{a a=A B}\right]$ :

- the aa-population grows exponentially fast,

- $n_{A A}\left(T^{a a=A B}\right)=\Theta\left(\varepsilon^{\gamma}\right)$,

- $n_{A B}\left(T^{a a=A B}\right)=\Theta\left(\sqrt{\varepsilon^{\gamma}}\right)$.

Proof. (1) Case 1: $T^{a A=A A} \geq T^{a a=A B}$.

Since in this case $n_{a A} \leq n_{A A}$ the arguments of Step 1.1 also hold here for the behaviour of the $A A$ - and $A B$-populations. Since only an increasing $A A$-population would stop the growth of $a a$, we see that the minorising process on $a a$, constructed in Step 1.1 before needs time $\Theta\left(\ln \left(\varepsilon^{-3 \gamma / 2}\right)\right)<T_{\varepsilon^{\gamma}}^{A A}$ to increase until $n_{A B}$.

(2) Case 2: $T^{a A=A A} \leq T^{a a=A B}$.

In this step $n_{A B} \geq n_{a a} \geq n_{a A}>n_{A A}$. The different=ce with Step 1.2 is that the $a a$-population is already bigger than the $a A$-population. Thus we can adapt the proof of Step 1.2 to this step with small changes. We have to ensure the growth of the $a a$-population until reaching $n_{A B}$. An increasing $a A$-population cannot stop the exponential growth of $n_{a a}$. We need to show that $n_{A B}$ does not start to grow 
to much. For this we have to estimate bounds on $n_{a A}$ and $n_{A A}$ again. The two following lemmata are similar to the ones in the step before (Lemma 4.26 and 4.27) but taking into account that now $n_{a a} \geq n_{a A}$.

Lemma 4.29. The AA-population is bounded from above by

$$
n_{A A} \leq \frac{2}{\bar{n}_{A}} n_{A B}^{2} .
$$

Proof. The proof is similar to the one of Lemma 4.8. We have to show that at the upper bound $\dot{n}_{A A} \leq \frac{4}{\bar{n}_{A}} \dot{n}_{A B} n_{A B}$. Observe that the lower bound on $\Sigma_{5}$ also holds here. We start by estimating a majorising process on $A A$ and by calculating the slope of it at the upper bound:

$$
\begin{aligned}
b_{A A} & \leq \frac{f}{\Sigma_{5}}\left(\frac{1}{2} n_{a A}+n_{A A}+\frac{1}{2} n_{A B}\right)^{2} \\
& =\frac{f}{\Sigma_{5}} n_{A A}\left(n_{a A}+n_{A A}+n_{A B}\right)+\frac{f}{4 \Sigma_{5}}\left(n_{a A}+n_{A B}\right)^{2}, \\
d_{A A} & \geq n_{A A}(f-\Theta(\Delta)), \\
\dot{n}_{A A} & \leq-n_{A A}(f-\Theta(\Delta))+\frac{f}{\Sigma_{5}} n_{A B}^{2} .
\end{aligned}
$$

The slope of this majorising process at the upper bound is estimated by $\dot{n}_{A A} \leq$ $-\frac{f-\Theta(\Delta)}{\bar{n}_{A}} n_{A B}^{2}<0$. Thus if we can show that the slope of a minorising process on $A B$ would be positive at the upper bound we are done:

$$
\begin{aligned}
& b_{A B} \geq f n_{A B}-\frac{f}{2 \Sigma_{6}} n_{A B}\left(2 n_{a a}+n_{a A}+n_{a B}+n_{A B}\right)+\frac{2 f}{\Sigma_{6}}\left(\frac{1}{2} n_{a A}+n_{A A}\right)\left(\frac{1}{2} n_{a B}+n_{B B}\right), \\
& d_{A B} \leq n_{A B}\left(f+\Theta\left(\Delta n_{A B}\right)\right), \\
& \dot{n}_{A B} \geq-\frac{5 f+\Theta(\Delta)}{2 \Sigma_{6}} n_{A B}^{2}+\frac{2 f}{\Sigma_{6}} n_{A A}\left(\frac{1}{2} n_{a B}+n_{B B}\right) .
\end{aligned}
$$

The slope of this process at the upper bound can be estimated by $\dot{n}_{A B} \geq \frac{3 f-\Theta(\Delta)}{4 \bar{n}_{B}} n_{A B}^{2}>$ 0 . This finishes the proof.

We proceed similarly for the upper bound on $a A$ :

Lemma 4.30. For $\eta<\frac{c}{2}$, the aA-population is bounded from above by

$$
n_{a A} \leq \frac{6 f}{\bar{n}_{A}(D-\Delta)} n_{A B}^{2}
$$

Proof. This time we have to show that at the upper bound $\dot{n}_{a A} \leq \frac{12 f}{\bar{n}_{A}(D-\Delta)} \dot{n}_{A B} n_{A B}$. We start by estimating a majorising process on $a A$ an by calculating the slope of it at the upper bound:

$$
\begin{aligned}
b_{a A} \leq & \frac{f}{2} n_{a A}+f n_{A A}+\frac{f}{2 \Sigma_{5}} n_{a A}\left(n_{a A}+2 n_{A A}+n_{a B}+n_{A B}\right) \\
& +\frac{f}{2 \Sigma_{5}} n_{a B}\left(2 n_{A A}+n_{A B}\right)+\frac{f}{\Sigma_{5}} n_{a a}\left(\frac{1}{2} n_{a A}+n_{A A}\right), \\
d_{a A} \geq & n_{a A}\left(f-\Theta(\Delta)-\eta n_{B B}\right) \geq \frac{f+D-\Theta(\Delta)}{2} n_{a A}, \\
\dot{n}_{a A} \leq & -\frac{D-\Theta(\Delta)}{2} n_{a A}+\frac{5 f+\Theta(\Delta)}{2 \bar{n}_{A}} n_{A B}^{2} .
\end{aligned}
$$

We used Proposition 4.21, Lemma 4.29 and $\eta<\frac{c}{2}$. The slope of this majorising process at the upper bound is estimated by $\dot{n}_{a A} \leq-\frac{f-\Theta(\Delta)}{2 \bar{n}_{A}} n_{A B}^{2}<0$. Thus if we can show that the slope of a minorising process on $A B$ would be positive at the upper bound we are done. We use the minorising process of $A B$ from before (cf.44.289) and estimate the slope which is given by $\dot{n}_{A B} \geq \frac{7 f-\Theta(\Delta)}{4 \bar{n}_{B}} n_{A B}^{2}>0$. This finishes the proof. 
Now we can estimate a majorising process on $A B$ and can bound it by $\dot{n}_{A B} \leq$ $-\Theta\left(n_{A B}^{2}\right)$ and $n_{A B}(t) \leq \frac{\Theta(1)}{\Theta\left(\varepsilon^{-\gamma / 2}\right)-\Theta(1) t}$. We have to construct a minorising process on aa. Since now $n_{A A} \leq n_{a A} \leq n_{a a}$ we can estimate:

$$
\begin{aligned}
& b_{a a} \geq \frac{f}{2} n_{a a}, \\
& d_{a a} \leq n_{a a}\left(D+\Delta+2 c n_{A B}\right), \\
& \dot{n}_{a a} \geq n_{a a}\left(\frac{f}{2}-D-\Theta(\Delta)\right) .
\end{aligned}
$$

Thus the $a a$-population grows exponentially fast $n_{a a}(t) \geq \Theta\left(\varepsilon^{\gamma}\right) e^{(f / 2-D-\Theta(\Delta)) t}$. The time $T_{\varepsilon^{\gamma / 2}}^{A B}$ the $A B$-population needs to exceed $\Theta\left(\varepsilon^{\gamma / 2}\right)$ is of order $\Theta\left(\varepsilon^{-\gamma / 2}\right)$ and the time $T_{\varepsilon^{\gamma / 2}}^{a a}$ the $a a$-population needs to reach $\Theta\left(\varepsilon^{\gamma / 2}\right)$ is of order $\Theta\left(\ln \varepsilon^{-\gamma / 2}\right)$. Thus $T_{\varepsilon^{\gamma / 2}}^{a a} \leq T_{\varepsilon^{\gamma / 2}}^{A B}$ and $T^{a a=A B}<\infty$.

\subsubsection{Step 3: Time interval $\left[T^{a a=A B}, T_{3}\right]$.}

Proposition 4.31. For $\eta<\frac{c}{2}$ we have $T_{3}<\infty$ and for all $t \in\left[T^{a a=A B}, T_{3}\right]$ :

- the aa-population grows exponentially fast,

- $n_{A A}\left(T_{3}\right)=\Theta\left(\varepsilon^{\gamma}\right)$,

- $n_{A B}\left(T_{3}\right)=\Theta\left(\sqrt{\varepsilon^{\gamma}}\right)$.

Proof. To ensure the exponential growth of $a a$ we have to consider the behaviour of the other processes as soon as $n_{a a} \geq n_{A B}$. Observe that the bound calculated in Lemma 4.29 takes over for this step unless if $n_{a A}>n_{A A}$ or $n_{a A} \leq n_{A A}$. We have to check again the upper bound on $a A$ :

Lemma 4.32. For $\eta<\frac{c}{2}$. the aA-population is bounded from above by

$$
n_{a A} \leq \frac{8 f}{\bar{n}_{A}(D-\Delta)} n_{A B}^{2}
$$

Proof. This time the competition of $a A$ with $a a$ contributes to its death-rate. We have to show that at the upper bound $\dot{n}_{a A} \leq \frac{16 f}{\bar{n}_{A}(D-\Delta)} \dot{n}_{A B} n_{A B}$. We start by estimating a majorising process on $a A$ an by calculating the slope of it at the upper bound:

$$
\begin{aligned}
b_{a A} \leq & \frac{f}{2 \Sigma_{3}} n_{a a} n_{a A}+f n_{A A}+\frac{f}{2 \Sigma_{5}} n_{a A}\left(n_{a A}+2 n_{A A}+n_{a B}+n_{A B}\right) \\
& +\frac{f}{2 \Sigma_{5}} n_{a B}\left(2 n_{A A}+n_{A B}\right)+\frac{f}{\Sigma_{6}} n_{a a}\left(\frac{1}{2} n_{a A}+n_{A A}\right), \\
d_{a A} \geq & n_{a A}\left(f-\Theta(\Delta)-\eta n_{B B}+c n_{a a}\right) \geq\left(\frac{f+D-\Theta(\Delta)}{2}+c n_{a a}\right) n_{a A}, \\
\dot{n}_{a A} \leq & -\left(\frac{D-\Theta(\Delta)}{2}+\frac{c(f-2 D)}{2(f-D)} n_{a a}\right) n_{a A}+\frac{5 f+\Theta(\Delta)}{2 \bar{n}_{A}} n_{A B}^{2} .
\end{aligned}
$$

We used Proposition 4.21, Lemma 4.29 and $\eta<\frac{c}{2}$. The slope of this majorising process at the upper bound is estimated by $\dot{n}_{a A} \leq-\frac{f-\Theta(\Delta)}{2 \bar{n}_{A}} n_{A B}^{2}<0$. Thus if we can show that the slope of a minorising process on $A B$ would be positive at the upper bound we are done:

$$
\begin{aligned}
& b_{A B}=\frac{2 f}{\Sigma_{5}}\left(\frac{1}{2} n_{a A}+n_{A A}+\frac{1}{2} n_{A B}\right)\left(\frac{1}{2} n_{a B}+\frac{1}{2} n_{A B}+n_{B B}\right)-\frac{f}{\Sigma_{5} \Sigma_{6}} n_{a a}\left(\frac{1}{2} n_{a A}+n_{A A}\right)\left(\frac{1}{2} n_{a B}+\frac{1}{2} n_{A B}+n_{B B}\right) \\
& \geq \frac{f}{\Sigma_{5}} n_{A B}\left(\frac{1}{2} n_{a A}+n_{A A}+\frac{1}{2} n_{a B}+\frac{1}{2} n_{A B}+n_{B B}\right)+\frac{2 f}{\Sigma_{5}}\left(\frac{1}{2} n_{a A}+n_{A A}\right)\left(\frac{1}{2} n_{a B}+n_{B B}\right)-\frac{f}{\Sigma_{6}} n_{a a}\left(\frac{1}{2} n_{a A}+n_{A A}\right), \\
& d_{A B} \leq n_{A B}\left(f+\Theta\left(n_{a A}+n_{A A}\right)\right), \\
& \dot{n}_{A B} \geq-\frac{4 f+\Theta(\Delta)}{\bar{n}_{A}} n_{A B}^{2}+\frac{f}{\Sigma_{5}} n_{a A}\left(\frac{1}{2} n_{a B}+n_{B B}\right)-\Theta\left(n_{A B} n_{a A}\right) .
\end{aligned}
$$


Estimation of the slope gives $\dot{n}_{A B} \geq \frac{2 f-\Theta(\Delta)}{\bar{n}_{A}} n_{A B}^{2}>0$. This finishes the proof.

We also need a majorising process on $A B$ to ensure that it does not grow too much. For this we use Proposition 4.21, Lemma 4.29 and Lemma 4.32.

$$
\begin{aligned}
b_{A B} & =\frac{2 f}{\Sigma_{5}}\left(\frac{1}{2} n_{a A}+n_{A A}+\frac{1}{2} n_{A B}\right)\left(\frac{1}{2} n_{a B}+\frac{1}{2} n_{A B}+n_{B B}\right)-\frac{f}{\Sigma_{5} \Sigma_{6}} n_{a a}\left(\frac{1}{2} n_{a A}+n_{A A}\right)\left(\frac{1}{2} n_{a B}+\frac{1}{2} n_{A B}+n_{B B}\right) \\
& \leq \frac{f}{\Sigma_{5}} n_{A B}\left(\frac{1}{2} n_{a A}+n_{A A}+\frac{1}{2} n_{a B}+\frac{1}{2} n_{A B}+n_{B B}\right)+\frac{2 f}{\Sigma_{5}}\left(\frac{1}{2} n_{a A}+n_{A A}\right)\left(\frac{1}{2} n_{a B}+n_{B B}\right), \\
d_{A B} & \geq n_{A B}\left(f-\Theta\left(n_{a A}+n_{A A}\right)\right), \\
\dot{n}_{A B} & \leq-\frac{f-\Theta(\Delta)}{\Sigma_{5}} n_{A B}^{2}+\frac{10 f}{\bar{n}_{A}} n_{A B}^{2} \leq \Theta\left(n_{A B}^{2}\right),
\end{aligned}
$$

Thus we see that the $a a$-population cannot disturb the behaviour of $A B$ much and we can estimate $\dot{n}_{A B} \leq-\Theta\left(n_{A B}^{2}\right)$ and $n_{A B}(t) \leq \frac{\Theta(1)}{\Theta\left(\varepsilon^{-\gamma / 2}\right)-\Theta(1) t}$. We show that the $a a$-population grows exponentially fast up until an $\varepsilon^{\gamma}$-neighbourhood of its equilibrium $\bar{n}_{a}$. Again we construct a minorising process:

$$
\begin{aligned}
& b_{a a} \geq n_{a a}\left(f-\Theta\left(\frac{n_{A B}^{2}}{n_{a a}}\right)\right), \\
& d_{a a} \leq n_{a a}\left(D+\Delta+c n_{a a}+\Theta\left(n_{A B}^{2}\right)\right), \\
& \dot{n}_{a a} \geq n_{a a}\left(f-D-\Delta-c n_{a a}-\Theta\left(\frac{n_{A B}^{2}}{n_{a a}}\right)\right) .
\end{aligned}
$$

This minorising process on $a a$ increases until an $\varepsilon^{\gamma}$-neighbourhood of $\bar{n}_{a}$. The time $T_{3}$ the $a a$-population needs to reach the $\varepsilon^{\gamma}$-neighbourhood of its equilibrium is of or$\operatorname{der} \Theta\left(\varepsilon^{\gamma} \ln \varepsilon^{\gamma}\right)$ and the time $T_{\varepsilon^{\gamma / 2}}^{A B}$ the $A B$-population needs to exceed $\Theta\left(\varepsilon^{\gamma / 2}\right)$ is of order $\Theta\left(\varepsilon^{-\gamma / 2}\right)$. Thus $T_{3} \leq T_{\varepsilon^{\gamma / 2}}^{A B}<\infty$.

4.5. Phase 4: Convergence to $p_{a B}=\left(\bar{n}_{a}, 0,0,0,0, \bar{n}_{B}\right)$. The Jacobian matrix of the field (2.24) at the fixed point $p_{a B}$ has the 6 eigenvalues: 0 (double), and $-(2 f-D),-(f-D+$ $\Delta),-(f-D-\Delta),-((f-D)(5 f-4 D)+f \Delta) /\left(4(f-D)+\eta \bar{n}_{B}\right)$ which are strictly negative under Assumptions $(C)$. Because of the zero eigenvalues, $p_{a B}$ is a non-hyperbolic equilibrium point of the system and linearisation fails to determine its stability properties. Instead, we use the result of the center manifold theory $(18,26)$ that asserts that the qualitative behaviour of the dynamical system in a neighbourhood of the non-hyperbolic critical point $p_{a b}$ is determined by its behaviour on the center manifold near $p_{a B}$.

Theorem 4.33 (The Local Center Manifold Theorem 2.12.1 in 26). Let $f \in C^{r}(E)$, where $E$ is an open subset of $\mathbb{R}^{n}$ containing the origin and $r \geq 1$. Suppose that $f(0)=0$ and $D f(0)$ has $c$ eigenvalues with zero real parts and s eigenvalues with negative real parts, where $c+s=n$. Then the system $\dot{z}=f(z)$ can be written in diagonal form

$$
\begin{aligned}
& \dot{x}=C x+F(x, y) \\
& \dot{y}=P y+G(x, y),
\end{aligned}
$$

where $z=(x, y) \in \mathbb{R}^{c} \times \mathbb{R}^{s}, C$ is a $c \times c$-matrix with c eigenvalues having zero real parts, $P$ is a $s \times s$-matrix with s eigenvalues with negative real parts, and $F(0)=G(0)=0, D F(0)=$ $D G(0)=0$. Furthermore, there exists $\delta>0$ and a function, $h \in C^{r}\left(N_{\delta}(0)\right)$, where $N_{\delta}(0)$ is the $\delta$-neighbourhood of 0 , that defines the local center manifold and satisfies:

$$
\operatorname{Dh}(x)[C x+F(x, h(x))]-P h(x)-G(x, h(x))=0,
$$


for $|x|<\delta$. The flow on the center manifold $W^{c}(0)$ is defined by the system of differential equations

$$
\dot{x}=C x+F(x, h(x)),
$$

for all $x \in \mathbb{R}^{c}$ with $|x|<\delta$.

The Local Center Manifold Theorem shows that the non-hyperbolic critical point $p_{a B}$ is indeed a stable fixed point and that the flow on the center manifold near the critical point approaches $p_{a B}$ with speed $\frac{1}{t}$. This can be seen as follows:

By the affine transformation $\left(n_{a a}, n_{B B}\right) \mapsto\left(n_{a a}-\bar{n}_{a}, n_{B B}-\bar{n}_{B}\right)$ we get a translated system $\tilde{F}(n)$ which has a critical point at the origin. The two eigenvectors corresponding to 0 eigenvalues of the Jacobian matrix of $\tilde{F}$ at the fixed point $(0,0,0,0,0,0)$ are

$$
E V_{1}=(0,0,0,0,1,0,-1) \text { and } E V_{2}=(0,0,0,0,-1,1,0)
$$

We perform a new change of variable to work in the basis of eigenvectors of $\tilde{F}(n)$. Let us call the new coordinates $x_{1}, \ldots, x_{6}$. Let $h\left(x_{1}, x_{2}\right)$ be the local center manifold. We shall look at its local shape near $(0,0)$ and expand it up to second order:

$$
h\left(x_{1}, x_{2}\right)=\left(\begin{array}{l}
\lambda_{3} x_{1}^{2}+v_{3} x_{1} x_{2}+\mu_{3} x_{2}^{2} \\
\lambda_{4} x_{1}^{2}+v_{4} x_{1} x_{2}+\mu_{4} x_{2}^{2} \\
\lambda_{5} x_{1}^{2}+v_{5} x_{1} x_{2}+\mu_{5} x_{2}^{2} \\
\lambda_{6} x_{1}^{2}+v_{6} x_{1} x_{2}+\mu_{6} x_{2}^{2}
\end{array}\right)+O\left(x^{3}\right) .
$$

We then substitute the series expansions into the center manifold equation 4.316 which gives us 12 equations for the 12 unknowns $\lambda_{3}, \ldots, \mu_{6}$. Substitution of the explicit second order approximation of the center manifold equation into (4.317) yields the flow on the local center manifold:

$$
\begin{aligned}
& \dot{x}_{1}=\frac{A_{1}}{B_{1}} x_{1} x_{2}+\frac{C_{1}}{D_{1}} x_{2}{ }^{2}+\frac{E_{1}}{F_{1}} x_{1}{ }^{2}+O\left(x^{3}\right) \\
& \dot{x}_{2}=\frac{A_{2}}{B_{2}} x_{1} x_{2}+\frac{C_{2}}{D_{2}} x_{2}{ }^{2}+\frac{E_{2}}{F_{2}} x_{1}{ }^{2}+O\left(x^{3}\right)
\end{aligned}
$$

where

$$
\begin{aligned}
A_{1}= & 3 c^{2} D f^{2}-c^{2} \Delta f^{2}-3 c^{2} f^{3} \\
B_{1}= & (D-\Delta-f)\left(4 c D^{2}-9 c D f+c \Delta f+5 c f^{2}-4 D^{2} \eta+4 D \Delta \eta+8 D \eta f-4 \Delta \eta f-4 \eta f^{2}\right) \\
C_{1}= & 12 c^{2} D^{3} f^{2}-4 c^{2} D^{2} \Delta f^{2}-39 c^{2} D^{2} f^{3}+12 c^{2} D \Delta f^{3}+42 c^{2} D f^{4}-c^{2} \Delta^{2} f^{3}-8 c^{2} \Delta f^{4} \\
& -15 c^{2} f^{5}+12 c D^{3} \eta f^{2}-16 c D^{2} \Delta \eta f^{2}-36 c D^{2} \eta f^{3}+4 c D \Delta^{2} \eta f^{2}+32 c D \Delta \eta f^{3} \\
& +36 c D \eta f^{4}-4 c \Delta^{2} \eta f^{3}-16 c \Delta \eta f^{4}-12 c \eta f^{5} \\
D_{1}= & 8(D-2 f)(D-f)(D-\Delta-f) \times \\
& \times\left(4 c D^{2}-9 c D f+c \Delta f+5 c f^{2}-4 D^{2} \eta+4 D \Delta \eta+8 D \eta f-4 \Delta \eta f-4 \eta f^{2}\right) \\
E_{1}= & c f, \quad F_{1}=2(-D+\Delta+f)
\end{aligned}
$$

and

$$
\begin{aligned}
& A_{2}=2 c^{2} D^{2} f-3 c^{2} D f^{2}+c^{2} f^{3}-2 c D^{2} \eta f+2 c D \Delta \eta f+4 c D \eta f^{2}-2 c \Delta \eta f^{2}-2 c \eta f^{3} \\
& B_{2}=(D-\Delta-f)\left(4 c D^{2}-9 c D f+c \Delta f+5 c f^{2}-4 D^{2} \eta+4 D \Delta \eta+8 D \eta f-4 \Delta \eta f-4 \eta f^{2}\right) \\
& C_{2}=-3 c D \eta f^{2}+c \Delta \eta f^{2}+3 c \eta f^{3} \\
& D_{2}=2(D-2 f)\left(4 c D^{2}-9 c D f+c \Delta f+5 c f^{2}-4 D^{2} \eta+4 D \Delta \eta+8 D \eta f-4 \Delta \eta f-4 \eta f^{2}\right) \\
& E_{2}=0, F_{2}=1 .
\end{aligned}
$$



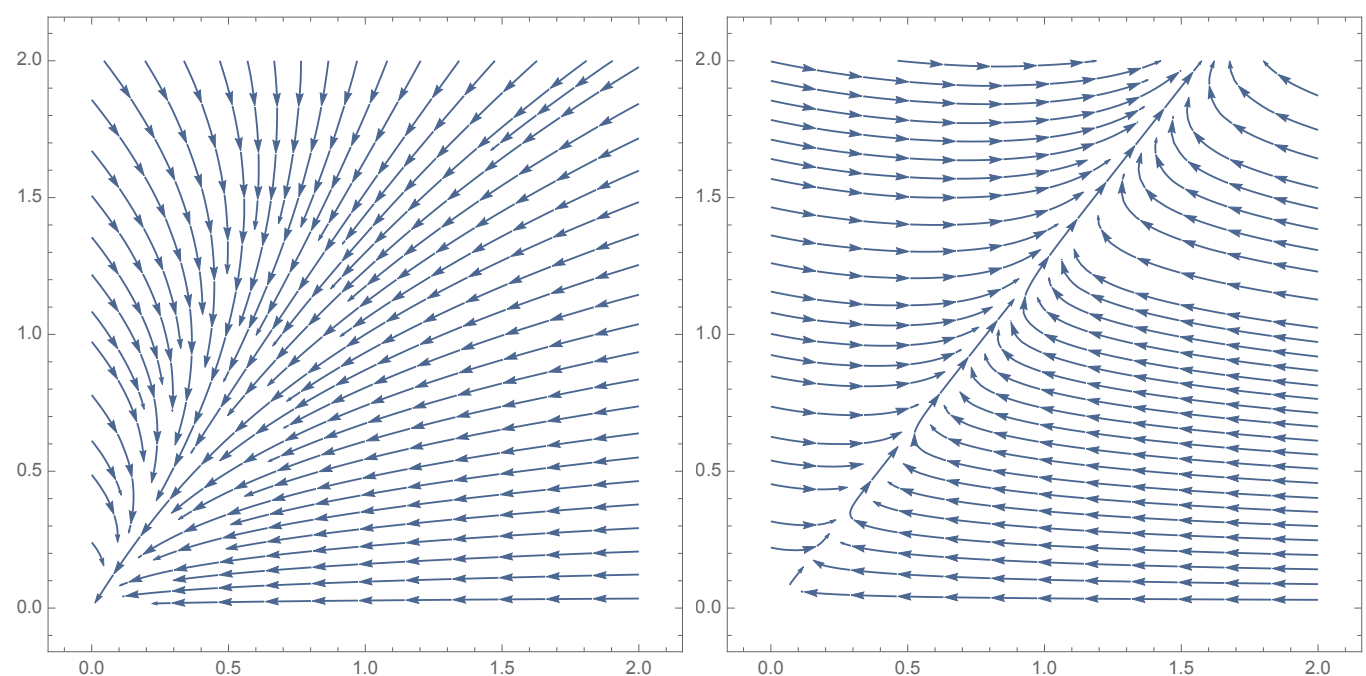

FIGURE 7. Flow of the dynamical system in the center manifold of the fixed point $p_{a B}$, for $\eta=0.02$ (left) and $\eta=0.6$ (right).

It is left to show that the above system flows toward the origin, at least for $\eta$ smaller than a certain constant. To do that, we perform another change of variables which allows us to work in the positive quadrant. We call the new coordinates (on the center manifold) $y_{1}$ and $y_{2}$, and the new field $\hat{F}$. Observe that it is sufficient to prove that the scalar product of the field with the position is negative. We thus consider the function

$$
s\left(y_{1}, y_{2}\right)=\left(\hat{F}\left(y_{1}, y_{2}\right),\left(y_{1}, y_{2}\right)\right)
$$

which is a quadratic form in $y_{1}$ and $y_{2}$. As the field $\hat{F}$ is homogeneous of degree 2 in its variables, it is enough to consider any direction given by $y_{2}=\lambda y_{1}$, and prove that $s\left(y_{1}, \lambda y_{1}\right)<0$ for all $\lambda>0$. As the expressions are so ugly, we work perturbatively in $f$ and consider it as large as needed. Observe that the numerator and the denominator of $s\left(y_{1}, \lambda y_{1}\right)$ are polynomials of degree 5 in $f$. We thus look at the coefficients in front of $f^{5}$ :

$$
s\left(y_{1}, \lambda y_{1}\right)=\frac{c y_{1}^{3}\left(c\left(16 \lambda^{3}+7 \lambda^{2}+16 \lambda+40\right)-4 \eta\left(5 \lambda^{3}+8 \lambda^{2}+8 \lambda+8\right)\right)}{64 \eta-80 c} f^{5}+\Theta\left(f^{4}\right)
$$

Observe that the denominator is always negative (because by our Assumptions $\eta \leq c$ ). The minimal value of the ratio

$$
r(\lambda):=\frac{16 \lambda^{3}+7 \lambda^{2}+16 \lambda+40}{4\left(5 \lambda^{3}+8 \lambda^{2}+8 \lambda+8\right)}
$$

is $r_{\text {max }} \simeq 0.593644$, thus, asymptotically as $f \rightarrow \infty$, the field is attractive for $\eta<c \cdot r_{\text {max }}$. Thus we see that $p_{a B}$ is a stable fixed point which is approached with speed $\frac{1}{t}$ as long as $\eta<c \cdot r_{\max }$.

\section{REFERENCES}

[1] M. Baar, A. Bovier, and N. Champagnat. From stochastic, individual-based models to the canonical equation of adaptive dynamics - In one step. Ann. Appl. Probab., online first, 2016.

[2] R. Bürger. The mathematical theory of selection, recombination, and mutation. Wiley Series in Mathematical and Computational Biology. John Wiley \& Sons, Ltd., Chichester, 2000. 
[3] N. Champagnat. A microscopic interpretation for adaptive dynamics trait substitution sequence models. Stochastic Process. Appl., 116(8):1127-1160, 2006.

[4] N. Champagnat, R. Ferrière, and G. Ben Arous. The Canonical Equation of Adaptive Dynamics: A Mathematical View. Selection, 2:73-83., 2001.

[5] N. Champagnat, R. Ferrière, and S. Méléard. From individual stochastic processes to macroscopic models in adaptive evolution. Stochastic Models, 24(suppl. 1):2-44, 2008.

[6] N. Champagnat and S. Méléard. Polymorphic evolution sequence and evolutionary branching. Probab. Theory Related Fields, 151(1-2):45-94, 2011.

[7] P. Collet, S. Méléard, and J. A. J. Metz. A rigorous model study of the adaptive dynamics of Mendelian diploids. J. Math. Biol., 67(3):569-607, 2013.

[8] C. Coron. Stochastic modeling of density-dependent diploid populations and the extinction vortex. $A d v$. in Appl. Probab., 46(2):446-477., 2014.

[9] C. Coron. Slow-fast stochastic diffusion dynamics and quasi-stationarity for diploid populations with varying size. J. Math. Biol., 72(1-2):171-202., 2016.

[10] C. Coron, S. Méléard, E. Porcher, and A. Robert. Quantifying the mutational meltdown in diploid populations. The American Naturalist, 181(5):623-36, 2013.

[11] J. F. Crow, M. Kimura, and Others. An introduction to population genetics theory. An introduction to population genetics theory., 1970.

[12] U. Dieckmann and R. Law. The dynamical theory of coevolution: a derivation from stochastic ecological processes. J. Math. Biol., 34(5-6):579-612., 1996.

[13] W. J. Ewens. Mathematical population genetics. I. Theoretical introduction, volume 27 of Interdisciplinary Applied Mathematics. Springer-Verlag, New York, second edition, 2004.

[14] R. Fisher. The correlation between relatives on the supposition of Mendelian inheritance. Trans. Roy. Soc. Edinb., 42:399-433., 1918.

[15] N. Fournier and S. Méléard. A microscopic probabilistic description of a locally regulated population and macroscopic approximations. Ann. Appl. Probab., 14(4):1880-1919, 2004.

[16] J. Haldane. A mathematical theory of natural and artificial selection. Part I. Trans. Camb. Phil. Soc., 23:19-41., 1924.

[17] J. Haldane. A mathematical theory of natural and artificial selection. Part II. Trans. Camb. Phil. Soc., Biol, Sci., 1:158-163., 1924.

[18] M. W. Hirsch, C. C. Pugh, and M. Shub. Invariant manifolds. Lecture Notes in Mathematics, Vol. 583. Springer-Verlag, Berlin-New York, 1977.

[19] J. Hofbauer and K. Sigmund. Adaptive dynamics and evolutionary stability. Appl Math Lett, 3(4):75$79,1990$.

[20] E. Kisdi and S. A. H. Geritz. Adaptive dynamics in allele space: Evolution of genetic polymorphism by small mutations in a heterogeneous environment. Evolution, 53:993-1008, 1999.

[21] P. Marrow, R. Law, and C. Cannings. The Coevolution of Predator-Prey Interactions: ESSS and Red Queen Dynamics. Proceedings of the Royal Society of London B: Biological Sciences, 250(1328):133141., 1992.

[22] J. Metz, R. Nisbet, and S. Geritz. How should we define "fitness" for general ecological scenarios? Trends in Ecology and Evolution, 7(6):198-202, 1992.

[23] J. A. J. Metz, S. A. H. Geritz, G. Meszéna, F. J. A. Jacobs, and J. S. van Heerwaarden. Adaptive dynamics, a geometrical study of the consequences of nearly faithful reproduction. In Stochastic and spatial structures of dynamical systems (Amsterdam, 1995), Koninklijke Nederlandse Akademie van Wetenschappen Eerste Reeks, 45, pages 183-231. North-Holland, Amsterdam, 1996.

[24] T. Nagylaki. Introduction to theoretical population genetics, volume 21 of Biomathematics. SpringerVerlag, Berlin, 1992.

[25] R. Neukirch and A. Bovier. Survival of a recessive allele in a Mendelian diploid model. J. Math. Biol., pages 1-43, nov 2016.

[26] L. Perko. Differential equations and dynamical systems, volume 7 of Texts in Applied Mathematics. Springer-Verlag, New York, 1991.

[27] S. Wright. Evolution in Mendelian populations. Genetics, 16:97-157., 1931.

[28] G. U. Yule. On the theory of inheritance of quantitative compound characters on the basis of Mendel's laws: a preliminary note. Spottiswoode \& Company, Limited, 1907. 
A. Bovier, Institut FÜR Angewandte Mathematik, Rheinische Friedrich-WilhelmsUniversität, ENDENicher AlleE 60, 53115 Bonn, GeRmany

E-mail address: bovier@uni-bonn.de

L. Coquille, Institut Fourier, UMR 5582 du CNRS, Université De GRENOble AlPEs, 100 RUE Des MathématiQues, 38610 GiÈRes, France

E-mail address: loren. coquille@univ-grenoble-alpes.fr

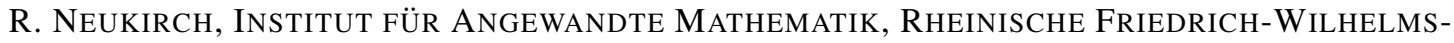
UniVERsitÄt, ENDENICHER AlleE 60, 53115 BonN, GERMANY

E-mail address: rebecca.neukirch@iam.uni-bonn.de 